\title{
The genus Enicospilus Stephens, 1835 (Hymenoptera, Ichneumonidae, Ophioninae) in Saudi Arabia, with twelve new species records and the description of five new species
}

\author{
Neveen S. GADALLAH ${ }^{1, *}$, Ahmed M. SOLIMAN ${ }^{2}$, \\ Pascal ROUSSE ${ }^{3} \&$ Hathal M. AL DHAFER ${ }^{4}$ \\ ${ }^{1}$ Entomology Department, Faculty of Science, Cairo University, Giza, Egypt. \\ ${ }^{2,4}$ Plant Protection Department, College of Food and Agriculture Sciences, King Saud University, \\ P.O. Box 2460, Riyadh 11451, Saudi Arabia. \\ ${ }^{2}$ Zoology Department, Faculty of Science (Boys), Al-Azhar University, \\ P.O. Box 11884, Nasr City, Cairo, Egypt. \\ ${ }^{3} 38$ rue des Primevères, 35160 Le Verger, France. \\ *Corresponding author: n_gadallah@hotmail.com \\ 2Email: amsoliman@ksu.edu.sa, ammsoliman@gmail.com \\ ${ }^{3}$ Email: rousse.pascal@wanadoo.fr \\ ${ }^{4}$ Email: hdhafer@ksu.edu.sa \\ ${ }^{1}$ urn:1sid:zoobank.org:author:49A46DDD-80CD-45FF-BC27-B37DBACF5E58 \\ ${ }^{2}$ urn:lsid:zoobank.org:author:638A9208-CA78-4161-91B4-914B531F8933 \\ ${ }^{3}$ urn:1sid:zoobank.org:author:B06C2640-700A-429B-AA2F-1BE09251C845 \\ ${ }^{4}$ urn:lsid:zoobank.org:author:6117A7D3-26AF-478F-BFE7-1C4E1D3F3C68
}

\begin{abstract}
The species of the genus Enicospilus Stephens, 1835 in Saudi Arabia are reviewed. Six species have previously been recorded from Saudi Arabia: E. brevicornis (Masi, 1939), E. capensis (Thunberg, 1822), E. nervellator Aubert, 1966, E. perlatus Shestakov, 1926, E. psammus Gauld \& Mitchell, 1978 and E. oculator Seyrig, 1935. Five new species are described and illustrated in this paper: Enicospilus arabicus Gadallah \& Soliman sp. nov., E. mirabilis Soliman \& Gadallah sp. nov., E. pseudoculator Gadallah \& Soliman sp. nov., E. shadaensis Gadallah \& Soliman sp. nov. and E. splendidus Rousse, Soliman \& Gadallah sp. nov. Twelve species are newly recorded for the fauna of Saudi Arabia, thus raising the total number to 23 species: E. bicoloratus Cameron, 1912, E. divisus (Seyrig, 1935), E. dubius (Tosquinet, 1896), E. grandiflavus Townes \& Townes, 1973, E. odax Gauld \& Mitchell, 1978, E. oweni Gauld \& Mitchell, 1976, E. pacificus (Holmgren, 1868), E. pallidus (Taschenberg, 1875), E. rundiensis Bischoff, 1915, E. senescens (Tosquinet, 1896), Enicospilus sp. 1 and Enicospilus sp. 2 cf. bicoloratus Cameron, 1912. The unknown male of E. odax is described for the first time. The COI barcodes of 17 specimens were sequenced, compared to the existing data and uploaded to the BOLD Systems database. An illustrated key and an annotated faunistic list of all species of Enicospilus in Saudi Arabia are also provided. Finally, we discuss the biogeographical and ecological significance of the Enicospilus fauna in Saudi Arabia.
\end{abstract}


Keywords. Saudi Arabia, Enicospilus, systematic, catalogue, biogeography.

Gadallah N.S., Soliman A.M., Rousse P. \& Al Dhafer H.M. 2017. The genus Enicospilus Stephens, 1835 (Hymenoptera, Ichneumonidae, Ophioninae) in Saudi Arabia, with twelve new species records and the description of five new species. European Journal of Taxonomy 365: 1-69. https://doi.org/10.5852/ejt.2017.365

\section{Introduction}

Saudi Arabia is a large arid land, covering the major part of the Arabian Peninsula, with an area of about $2250000 \mathrm{~km}^{2}$ (Aldhebiani \& Howladar 2013). It is characterized by different ecosystems and a large diversity of plant species. Components of the flora are a mixture of Asian, African and Mediterranean elements, and it is therefore considered to be one of the richest biodiversities in the Arabian Peninsula (Aldhebiani \& Howladar 2013). There is always a close correlation between insect and plant diversity (El-Moursy et al. 2001).

Biogeographically, Saudi Arabia as a whole is on the frontier between the Palaearctic and Afrotropical regions, the Arabian Desert being a strong and forbidding ecological barrier. The southwestern region of Saudi Arabia (including Al Baha and Asir provinces) is, however, considered to be more closely related to the Afrotropical region (Hölzel 1998). Its floristic diversity and richness are the highest in Saudi Arabia. The flora has strong affinities with parts of Africa, particularly East Africa. The Juniperus woodland present in the region under study is also well known in Eritrea, Somalia, Ethiopia, Kenya and Tanzania, and is situated at almost the same altitude (Zohary 1973).

The genus Enicospilus Stephens, 1835 is one of the largest genera of Ichneumonidae and the most speciose in the subfamily Ophioninae (Townes 1971; Gauld 1985; Bordera et al. 1987; Gracía 2011). It encompasses more than 700 species worldwide (Yu et al. 2012; Rousse \& Villemant 2012; Rousse \& van Noort 2014), but most of them are found in tropical or subtropical regions (Townes 1971; Gauld 1985; Gauld \& Mitchell 1978, 1981; Gauld 1988; Rousse et al. 2016). Only a small number of species is present in north temperate regions where Ophion Fabricius, 1798 is the predominant genus (Broad \& Shaw 2016).

The genus Enicospilus was first proposed by Stephens (1829) in his catalogue of British insects, with a single species, E. simulator, which is considered to be a nomen nudum because it was published without an accompanying description. The genus was proposed again by Stephens (1835) with a coloured plate. Several attempts have been made to subdivide the genus into a number of smaller genera (e.g., Kriechbaumer 1901; Szépligeti 1905; Seyrig 1935), but these are no longer accepted because they were based on characters which subsequently proved to be homoplastic (Gauld 1985; Rousse et al. 2016).

Species of this genus are usually very slender. They are characterized by the combination of the occluded spiracular sclerite together with nearly always strongly tapered and twisted mandibles (Rousse et al. 2016). Like many other nocturnal Ichneumonoidea they usually exhibit a characteristic facies (Gauld \& Huddleston 1976), i.e., they are moderate to large sized wasps with long slender antennae and enlarged ocelli, and with pale colouration, although a minority of species of Enicospilus are partly to totally dark in colour. In addition, the fore wing disco-submarginal cell almost always has a small to large hairless area below the base of the radial cell, a "fenestra", which usually contains one or more alar sclerites. These sclerites are of great taxonomic help, but have little phylogenetic relevance.

Relatively little is known about the biology of Enicospilus species. They are known as primarily nocturnal parasitoids parasitizing a large variety of lepidopteran larvae. Most species seem to attack 
larvae that are free-living caterpillars; the few with long ovipositors seem to attack larvae mining stems (Townes 1971; Gauld 1985; Yu et al. 2012).

No study has so far focused on the taxonomy of Enicospilus in Saudi Arabia. The genus is known to occur in the other countries of the Arabian Peninsula: seven species in the United Arab Emirates and eight in Yemen, while none has been recorded in Kuwait or Oman (Gauld \& Mitchell 1978; Yu et al. 2012; Wahl 2014). In Saudi Arabia, six species have been reported so far: E. brevicornis (Masi, 1939), E. capensis (Thunberg, 1822), E. nervellator Aubert, 1966, E. oculator Seyrig, 1935, E. perlatus Shestakov, 1926 and E. psammus Gauld \& Mitchell, 1978 (Gauld \& Mitchell 1978; Horstmann 1981; Yu et al. 2012). Five new species are described in the present work and twelve additional species are newly reported, increasing to 23 the total number of species known from Saudi Arabia. An illustrated key is provided and an annotated faunistic list is detailed and then discussed from a biogeographical point of view.

\section{Material and methods}

\section{Biogeography and ecology of the prospected localities}

The specimens were collected mainly in Central (Riyadh) and Southwestern (Al Baha and Asir) Saudi Arabia (Fig.1). The geographical data of the localities are detailed in Appendix 1. Fig. 2 illustrates the variety of the prospected habitats in these localities. Sampling was done by means of light traps and sweep nets, the collected specimens were pinned directly for further study.

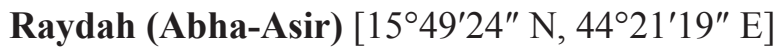

Lying at the border of the Somalia-Masai and Afromontane archipelago-like regional centres of endemism, this locality contains elements of both zones (Ghazanfar \& Fischer 1998). It covers only $933 \mathrm{~km}^{2}$ (http://www.birdsofsaudiarabia.com/2012/11/raydah-escarpment-reserve-abha-html). It protects the last remnants of dense juniper forest found in southwestern Yemen and across the Arabian Peninsula. It is dominated by: Juniperus procera Hochst. (Cupressaceae), Nuxia oppositifolia Hochst. (Stilbaceae), Maesa lanceolata Forssk. (Myrsinaceae), Celtis africana N.L.B Burm. (Cannabaceae), Teclea nobilis (Rutaceae), Tarchonanthus camphorates Linnaeus (Asteraceae) (below 1700 m), Ficus spp. (Moraceae), Buddleja polystachya Fresen. (Buddlejaceae), Ziziphus spina-christi (Linnaeus) (Rhamnaceae), Aloe sabaea Schweinf. (Xanthorrhoeaceae) and other aloes which dominate an unusual succulent community (below $1550 \mathrm{~m}$ ). Acacia etbaica Schweinf. (Fabaceae) is also found on the lowest slopes; among which grow other rare succulents such as Caralluma and Ceropegia spp. (Apocynaceae). Also found there are Aloe gracilis Lam. (Asphodelaceae), Ceropegia aristolochiodes Decne (Apocynaceae) and others that are endemic or near-endemic species. This is in addition to 25 other plant species that are rare in Saudi Arabia (Ghazanfar \& Fischer 1998).

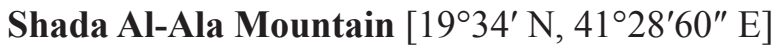

This is a natural protectorate in southwestern Al Baha province. It is located at an elevation of $514 \mathrm{~m}$ above sea level (http://www.getamap.net/maps/saudi_arabia/saudi_arabia_(general)/_shadaalala_ jabal/). It has a high diversity of plants including Albizia lebbeck (Linnaeus) (Fabaceae), Solenosteniom sp. (Lamiaceae), Juniperus procera Höchst. (Cupressaceae), Santalum spp. (Santalaceae), Pimpinella anisum Linnaeus (Apiaceae), Rhamnus frangula Linnaeus (Rhamnaceae), Opuntia ficus-indica (Linnaeus), Cactus sp. (Cactaceae), Ricinus communis Linnaeus (Euphorbiaceae), Olea europaea africana (Mill.) (Oleaceae), Prunus dulcis (Mill.) (Rosaceae), Maerua crassifolia Forssk. (Capparaceae), Pandarius tectorius Parkinson (Pandanaceae), Panicum turgidum Forssk. (Poaceae), Coffea arabica Linnaeus, Breonadia salicina (Vahl) (Rubiaceae), Haloxylon salicornicum (Mosq.) (Amaranthaceae), Lycium shawii Roem. \& Schult. (Solanaceae) and Acacia spp. (Fabaceae) (Sharaf et al. 2014). 
Al-Harmalyiah $\left[24^{\circ} 15.78^{\prime} \mathrm{N}, 45^{\circ} 12.45^{\prime} \mathrm{E}\right]$

At a distance of $165 \mathrm{~km}$ from Riyadh, this is an unfenced area where grazing, camping and vehicles are not controlled. It is characterized by the following kinds of vegetation: Haloxylon salicornicum (Moq.) (Amaranthaceae), Pulicaria undulata (Linnaeus) (Asteraceae), Convolvulus hystrix Vahl (Convolvulaceae), Rhazya stricta Dacne, Calotropis procera (Aiton) (Apocynacaea) and other seasonal herbs (Al Mobdel 2001).
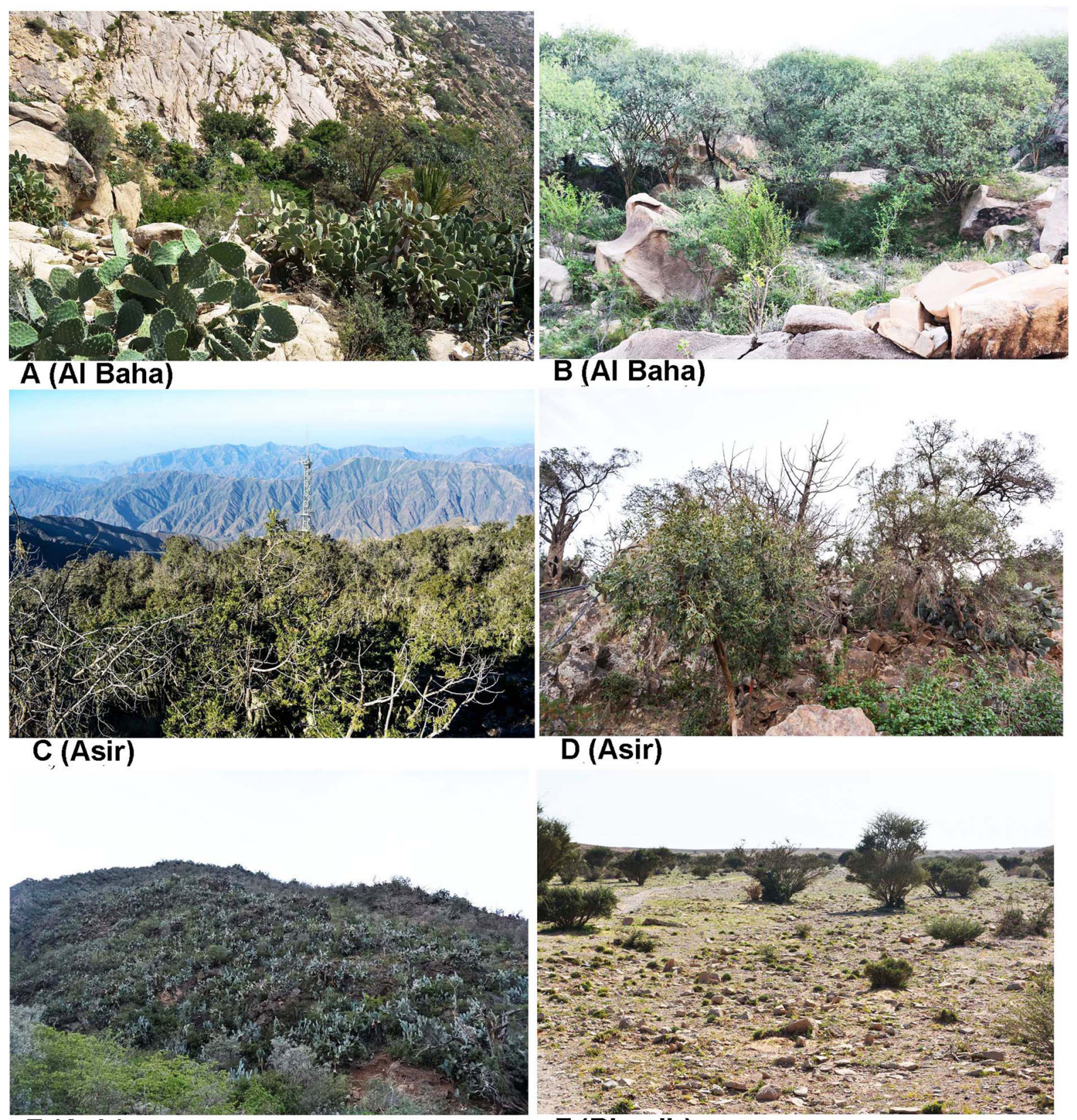

\section{E (Asir)}

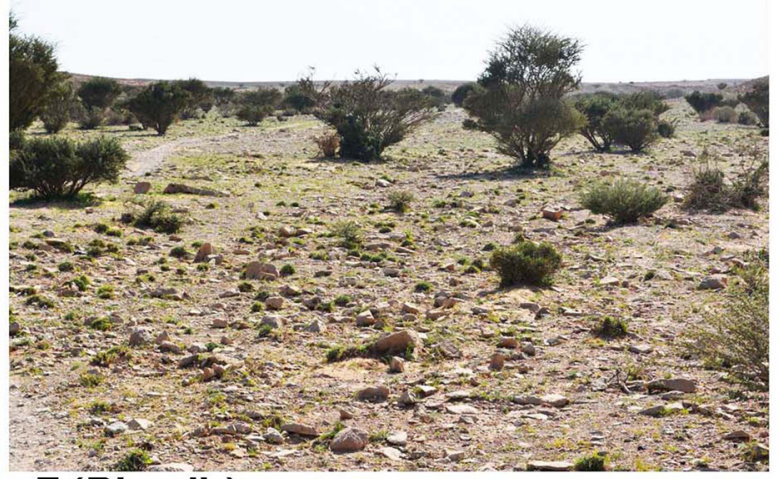

F (Riyadh)

Fig. 1. Examples of habitats where specimens of Enicospilus Stephens, 1835 were collected. A-B. Shada Al-Ala Mountain (Al Baha). C-E. Raydah (Asir). F. Rawdet Al Harmalyiah (Riyadh). 


\section{Depositories}

NHMUK $=$ Natural History Museum, London, United Kingdom (Gavin Broad)

EFC = Efflatoun Bey Collection, Entomology Department, Faculty of Science, Cairo University, Egypt (Neveen S. Gadallah)

KSMA = King Saud University Museum of Arthropods, Plant Protection Department, College of Food and Agriculture Sciences, King Saud University, Riyadh, Saudi Arabia (Hathal M. Al-Dhafer)

PPDD = Plant Protection Institute, Ministry of Agriculture, Giza, Egypt

\section{Pictures}

Photographic images were taken using a Canon EOS 70D Camera attached to a LEICA MZ 125 stereo microscope. Individual source images were then stacked using HeliconFocus v6.22 (HeliconSoft Ltd) extended depth of field software. Measurements of the different parts were made with the help of an ocular micrometer. Further image processing was done using the software Adobe Photoshop CS5.1 (ver. 12.1x32) and Adobe Photoshop Lightroom 5.2 Final (64 bit) [ChingLiu]. The distribution of the prospected sites (Fig. 2) is plotted using DIVA-GIS (ver.7.17).

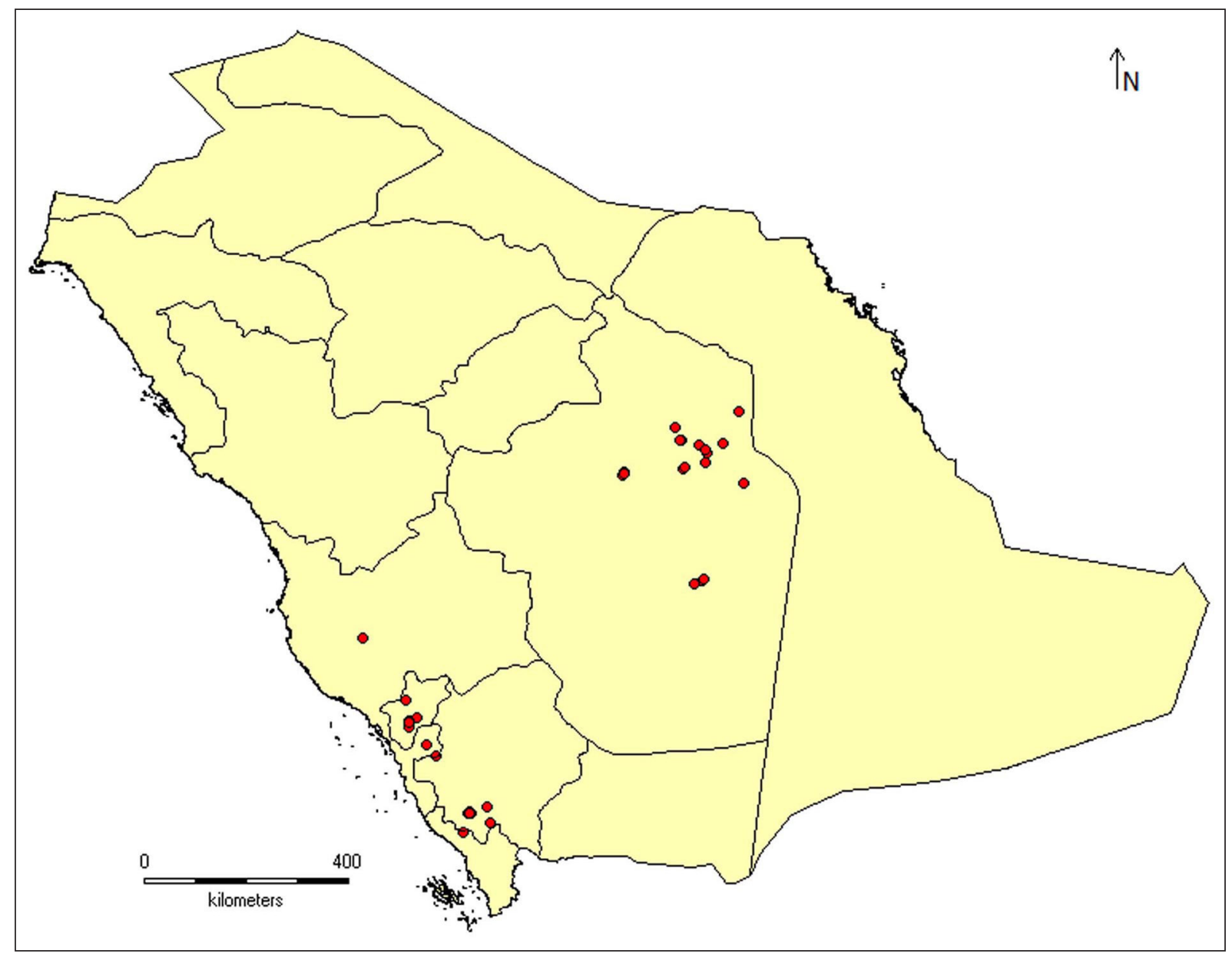

Fig. 2. Distributional map of collection localities in Saudi Arabia. 


\section{Terminology}

Morphological terms follow Gauld \& Mitchell (1978) and descriptions follow Rousse \& van Noort (2014) to facilitate comparison. Body-sculpture terminology follows Harris (1979). The following abbreviations are used (indices after Gauld \& Mitchell 1978; Rousse \& van Noort 2014):

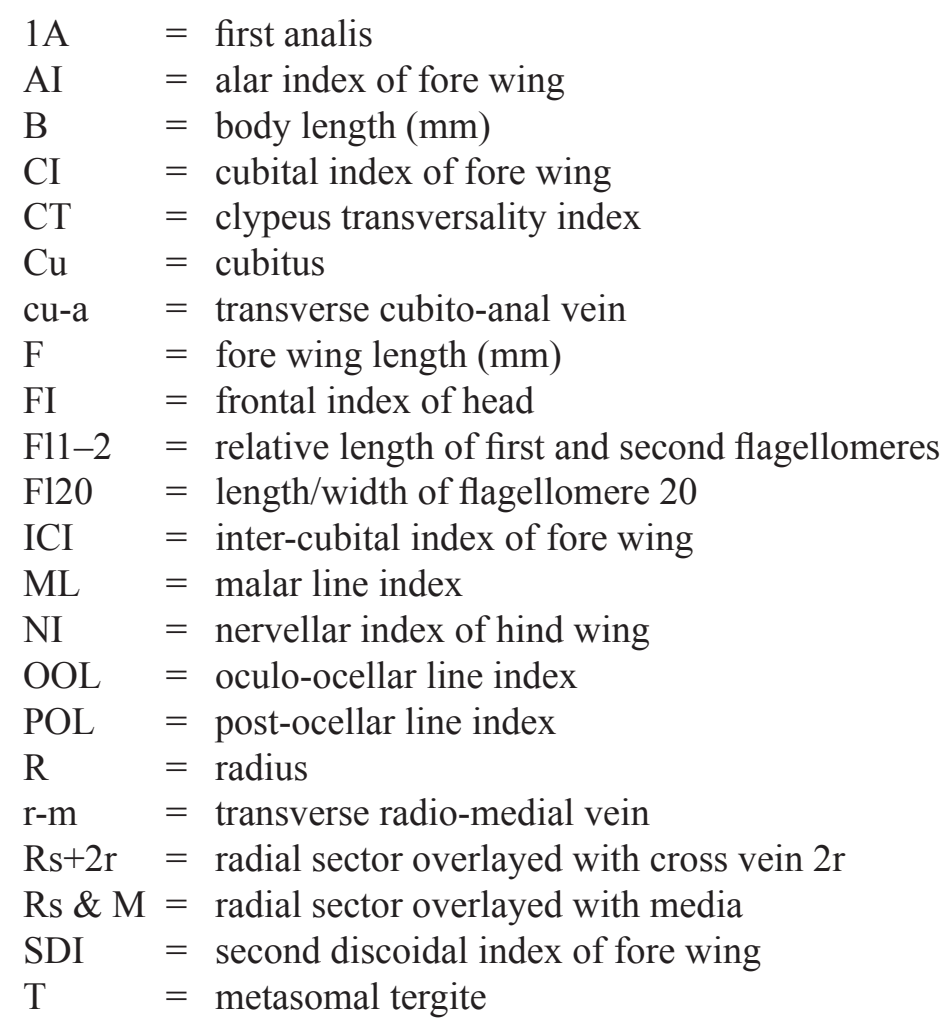

\section{Barcoding}

Seventeen specimens were barcoded and compared with existing data. DNA was extracted from one hind leg using the DNeasy Blood \& Tissue Kit (Qiagen) following the standard manufacturer protocol. $\mathrm{COI}(\mathrm{HCO} / \mathrm{LCO})$ was then amplified using the Taq PCR Core Kit (Qiagen): denaturation $94^{\circ} 2 \mathrm{mn}, 35$ cycles $94^{\circ} 30 \mathrm{~s}, 44^{\circ} 30 \mathrm{~s}$ and $72^{\circ} 60 \mathrm{~s}$, final elongation $72^{\circ} 5 \mathrm{mn}$. All PCR products were subsequently checked on agarose gel and sequenced with an ABI 3130 automated capillary DNA sequencer (Applied Biosystems). The sequences were eventually uploaded on the BOLD Systems database (Ratnasingham \& Hebert 2007). The registration references for the database are listed in Appendix 2.

\section{Results}

Class Hexapoda Blainville, 1816

Order Hymenoptera Linnaeus, 1758

Suborder Apocrita Latreille, 1810

Superfamily Ichneumonoidea Latreille, 1802

Family Ichneumonidae Latreille, 1802

Subfamily Ophioninae Shuckard, 1840

Genus Enicospilus Stephens, 1835

A total of 498 specimens of Enicospilus representing 23 species were collected and identified with the keys of Gauld \& Mitchell (1978) and Rousse \& van Noort (2014). Some uncertain identifications 
were confirmed with the help of Gavin Broad (NHMUK). All our sequences and related pictures were uploaded to the BOLD database, and the identification numbers are detailed in the relevant descriptions (see below). The DNA extraction was unsuccessful for E. senescens (Tosquinet, 1896) and not enough material was available at the moment of extraction of E. bicoloratus Cameron, 1912, E. perlatus, E. psammus, Enicospilus sp. 1 and sp. 2. Six of the 17 COI barcodes significantly matched (>97\% similarity) with databased sequences, all of which were registered as unidentified Enicospilus spp. in the BOLD database. These were the barcodes extracted from specimens morphologically identified as E. capensis, E. grandiflavus Townes \& Townes, 1973, E. oculator, E. pacificus (Holmgren, 1868), E. pallidus (Taschenberg, 1875) and E. shadaensis sp. nov.

Five new species are described herein: E. arabicus Gadallah \& Soliman sp. nov., E. mirabilis Soliman \& Gadallah sp. nov., E. pseudoculator Gadallah \& Soliman sp. nov., E. shadaensis Gadallah \& Soliman sp. nov. and E. splendidus Rousse, Soliman \& Gadallah sp. nov. In addition, this paper documents twelve species newly recorded from Saudi Arabia. Most of the reported species are typical of the Afrotropical region rather than the Palaearctic region, especially those collected from Al Baha and Asir provinces (southwestern Saudi Arabia). Of the Saudi species of Enicospilus, 86.9\% are Afrotropical, $8.7 \%$ are Palaearctic and $13 \%$ are both (E. capensis, E. psammus, E. pseudoculator sp. nov.) (Table 1). Interestingly, two species reported from Yemen (E. justus and E. expeditus) (Gauld \& Mitchell 1978) were not collected in the present study from South Western Saudi Arabia. An explanatory hypothesis might be that these two species were collected only in the high mountains in Yemen. As a consequence, we can not ascertain their presence in Saudi Arabia and did not include them in the key.

\section{Key to the species of Enicospilus in Saudi Arabia}

1. Disco-submarginal cell of fore wing without any alar sclerites (Figs 25C, 27B) ......................... 2

- Disco-submarginal cell of fore wing with one or more alar sclerites 3

2. Rs $+2 \mathrm{r}$ strongly sinuate proximally, fenestra relatively small, AI $>1$ (Fig. 27B); antenna with more than 65 flagellomeres

E. senescens (Tosquinet, 1896)

- Rs+2r straighter proximally (Fig. 25C), fenestra larger, AI $<1$; antenna with fewer than 65 flagellomeres

E. oweni Gauld \& Mitchell, 1976

3. Mesosoma interspersed with ivory markings (Figs 3H, 4B, 4G, 5B, 5C); antenna relatively short with fewer than 55 flagellomeres

- Mesosoma uniformly coloured, without ivory markings; antenna variable, usually with more than 55 flagellomeres

4. Fore wing with one proximal sclerite, central sclerite totally absent (Figs 26C, 27C, 28A) …...... 5

- Fore wing with one proximal and one central sclerite (Figs 24B, 25A) ........................................ 7

5. Proximal sclerite very weakly sclerotized (Fig. 28A); outer mid tibial spur very short, less than $0.4 \times$ as long as inner spur (Fig. 33C).

E. splendidus Rousse, Soliman \& Gadallah sp. nov.

- Proximal sclerite fully sclerotized (Figs 26C, 27C); outer mid tibial spur usually longer 6

6. Body dark reddish brown (Fig. 5B); proximal sclerite dark brown to black, triangular, CI $>0.2$ (Fig. 27C); face narrow, $1.5 \times$ higher than wide (Fig. 8F); moderately large species $(\mathrm{B}>27$, $\mathrm{F}>12)$ E. shadaensis Gadallah \& Soliman sp. nov.

- Body lighter orange to brown (Fig. 4G); proximal sclerite bright red and dome-shaped, CI $<0.2$ (Fig. 26C); face subquadrate, $1.1 \times$ higher than wide (Fig. 8C); smaller species $(\mathrm{B}<19$, $\mathrm{F}<12)$

E. pseudoculator Gadallah \& Soliman sp. nov. 
7. Metasoma interspersed with ivory markings (Fig. $3 \mathrm{H}$ ); central sclerite uniformly sclerotized (Fig. 24B); median flagellomeres stout $(\mathrm{Fl} 20<1.7)$........E. mirabilis Soliman \& Gadallah sp. nov.

- Metasoma without ivory markings (Fig. 4B); central sclerite weakly sclerotized proximally (Fig. 25A); median flagellomeres more slender $($ Fl20 > 1.7) E. oculator Seyrig, 1935

8. Proximal sclerite acutely arrow-shaped, central sclerite very weakly sclerotized (Fig. 25B); moderately to very large species (B 16-30, F 14-20)

- Proximal sclerite obviously different, central sclerite present or absent; usually smaller species ... 9

9. Central sclerite totally absent, without even a faint trace (Figs 22A, 22C, 23C, 24A) ................. 10

- Central sclerite present (e.g., Figs 22B, 23B, 26A, 26B, 28C) though sometimes weakly sclerotized (e.g., Figs 23A, 24C, 28C)

10. Pale yellow species (Figs 3A, 3G)

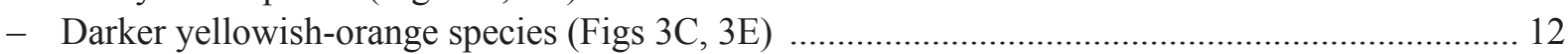

11. Proximal sclerite obtusely angled without distal extension, ICI $>0.6, \mathrm{CI}<0.3$ (Fig. 24A); propodeum coarsely and concentrically striate (Fig. 19A); clypeus flat in profile; metasoma darkened dorsally and apically (Fig. 3G); large species $(\mathrm{B}>25, \mathrm{~F}>15)$

E. grandiflavus Townes \& Townes, 1973

- Proximal sclerite comma-shaped with very long distal extension, ICI $<0.6$, CI $>0.3$ (Fig. 22A); integument is almost longitudinally striate just behind the basal transverse carina of propodeum (Fig. 18A); clypeus very strongly convex in profile (Fig. 10A); metasoma uniformly yellow without any dark markings (Fig. 3A); smaller species $(\mathrm{B}<25, \mathrm{~F}<15)$

E. arabicus Gadallah \& Soliman sp. nov.

12. Antenna moderately long with more than 50 flagellomeres, median ones strongly elongate (F120 > 2); metapleuron weakly convex, without punctures, and longitudinally striate

E. dubius (Tosquinet, 1896)

- Antenna short with fewer than 50 flagellomeres, median ones stout $(\mathrm{Fl} 20<2)$; metapleuron strongly convex, puncto-striate and longitudinally striate

E. brevicornis (Masi, 1939)

13. Central sclerite subdivided and U-shaped (Fig. 23B)

E. divisus (Seyrig, 1935)

- Central sclerite not elongate, obviously different

14. Proximal sclerite rather narrow, comma-shaped with very long distal extension, central sclerite uniformly sclerotized (Fig. 26A)

E. pacificus (Holmgren, 1868)

- Proximal sclerite different, more or less triangular sometimes with a faint distal extension (e.g., Figs 22B, 23A, 26B, 28C); central sclerite sometimes weakly sclerotized (e.g., Figs 23A, 24C, 28C)

15. Central sclerite crescent-shaped, weakly sclerotized proximally, and large with maximal length larger than distance to Rs+2r (Fig. 26B); pale yellow species (Fig. 4F)

E. pallidus (Taschenberg, 1875)

- Central sclerite smaller and more or less circular (e.g., Figs 22B, 23A, 28B), sometimes hardly sclerotized proximally and/or distinctly elongate (Figs 24C, 27A) but never crescent-shaped; usually darker yellowish-orange species 16

16. Very small species (F 6-9), with characteristic alar sclerites (Fig. 34A)

E. psammus Gauld \& Mitchell, 1978

- Larger species (F nearly always $>10$ ), with different alar sclerites 
17. Outer mid (and sometimes hind) tibial spur(s) very short, less than $0.4 \times$ as long as inner spur(s) (Figs 33A); central sclerite longitudinal and weakly sclerotized (Fig. 24C)

E. nervellator Aubert, 1966

- Outer mid and hind tibial spurs longer; central sclerite variable 18

18. Mandible with a long piliferous furrow from dorsal base to between teeth (Fig. 10D) 19

- Mandible without such a distinct piliferous furrow (e.g., Fig. 13B) 20

19. Mandible with upper tooth less than twice the length of lower tooth (Fig. 6B), bare or sparsely setose on outer margin (Fig. 6B); central sclerite small, circular, and uniformly sclerotized (Fig. 22B)

E. bicoloratus Cameron, 1912

- Mandible with upper tooth more than twice the length of lower tooth (Fig. 6D), with dense setae on outer margin (Figs 6D, 10D); central sclerite not uniformly sclerotized (Fig. 23A)

E. capensis (Thunberg, 1822)

20. Central sclerite small, shortly elongate and totally translucent (Fig. 34B); hind wing with Cu \& cu-a (nervellus) intercepted around middle (NI 1.0-1.2)

E. perlatus Shestakov, 1926

- Central sclerite circular to distinctly elongate, pigmented at least distally (Figs 27A, 28B-C); hind wing with $\mathrm{Cu} \& \mathrm{cu}-\mathrm{a}$ (nervellus) intercepted far below middle $(\mathrm{NI}>1.6)$

21. Central sclerite moderately to very long and curved toward base of wing, proximal sclerite dark brown (Fig. 27A)

E. rundiensis Bischoff, 1915

- Central sclerite more or less circular, proximal sclerite lighter (Figs 28B-C) 22

22. Central sclerite weakly sclerotized proximally (Fig. 28C); mesopleuron closely punctate without longitudinal striations (Fig. 17C) Enicospilus sp. 1

- Central sclerite fully sclerotized (Fig. 28B); mesopleuron puncto-striate

Enicospilus sp. 2 cf. bicoloratus Cameron, 1912

Enicospilus arabicus Gadallah \& Soliman sp. nov. urn:1sid:zoobank.org:act:68191502-FC11-4BBF-8A0E-DA1FAB4C4198

Figs 3A, 6A, 10A, 14A, 18A, 22A, 29A

\section{Diagnosis}

Moderately sized and yellow overall, including antennae and legs, pterostigma yellow, bordered posteriorly with black; mandible hardly twisted; clypeus strongly convex in profile, ventral margin blunt and in-turned; face $1.2 \times$ as high as wide; antenna with 58 flagellomeres; mesoscutum with exceptionally long notauli distinct over its entire length; mesopleuron and metapleuron nearly smooth, with very slight fine rugosity ventrally; propodeum nearly smooth anteriorly, coarsely rugose-reticulate posteriorly; proximal sclerite comma-shaped with very long distal extension.

\section{Etymology}

Named in reference to the country of Saudi Arabia, where the type specimen was collected.

\section{Type material}

\section{Holotype}

SAUDI ARABIA: , Asir, Abha, Raydah, light trap 1, $18^{\circ} 12.265^{\prime} \mathrm{N}, 42^{\circ} 24.744^{\prime} \mathrm{E}$, alt. $2820 \mathrm{~m}, 26$ Aug. 2014, leg. Al Dhafer et al. (KSMA). 
Table 1. Comparison of local and biogeographical distributions of species of Enicospilus Stephens, 1835 collected in Saudi Arabia. All areas but Riyadh are considered to be part of the Afrotropical region, Riyadh is more related to the Palaearctic region (Hölzel 1998).

\begin{tabular}{|c|c|c|}
\hline Species & $\begin{array}{l}\text { Biogeography } \\
\text { (Yu et al. 2012) }\end{array}$ & $\begin{array}{c}\text { Saudi Arabia } \\
\text { (present data; Gauld \& Mitchell 1978; Horstmann 1981) }\end{array}$ \\
\hline E. arabicus sp. nov. & - & Abha \\
\hline E. bicoloratus & Afrotropical & Al Baha, Asir \\
\hline E. brevicornis & Afrotropical & Abha, Asir \\
\hline E. capensis & Afrotropical, Oriental, Australasian & Abha, Al Baha, Asir, Riyadh \\
\hline E. divisus & Afrotropical & Asir \\
\hline E. dubius & Afrotropical & Asir \\
\hline E. grandiflavus & Afrotropical & Abha, Al Baha, Asir \\
\hline E. mirabilis sp. nov. & - & Abha, Al Baha, Asir \\
\hline E. nervellator & Palaearctic & Asir*, Riyadh \\
\hline E. oculator & Afrotropical & Al Baha \\
\hline E. odax & Afrotropical** & Al Baha, Asir \\
\hline E. oweni & Afrotropical & Asir \\
\hline E. pacificus & Afrotropical & Al Baha, Asir, Riyadh \\
\hline E. pallidus & Afrotropical, Oriental & Al Baha \\
\hline E. perlatus & Palaearctic & Mekka, Riyadh \\
\hline E. psammus & Afrotropical & Abian, Al Lith, Asir, Qunfida \\
\hline E. pseudoculator sp. nov. & - & Al Baha, Asir, Riyadh \\
\hline E. rundiensis & Afrotropical & Al Baha, Asir \\
\hline E. senescens & Afrotropical & Al Baha, Asir \\
\hline E. shadaensis sp. nov. & Afrotropical ${ }^{* * *}$ & Al Baha, Asir \\
\hline E. splendidus sp. nov. & - & Riyadh \\
\hline E. sp. 1 & - & Asir \\
\hline E. sp. 2 cf. bicoloratus & - & Al Baha \\
\hline
\end{tabular}

* One specimen collected in Asir, 237 in Riyadh. ** Restricted to Yemen. *** According to Enicospilus sp. 4 distribution in Gauld \& Mitchell 1978

\section{Paratypes}

SAUDI ARABIA: 1 ㅇ, Raydah (Abha), light trap 1, 5 Sep. 2015, leg. Al Dhafer et al. (KSMA); 1 , Raydah (Abha), light trap 4, 6 Jun. 2014, leg. Al Dhafer et al. (KSMA); 1 q, Raydah (Abha), light trap 9, 26 Aug. 2014, leg. Al Dhafer et al. (KSMA).

\section{Description}

Female (5 specimens)

Measurements. B 19-20; F 13-14; ML 0.4; CT 1.6; OOL 0.1; POL 1.1; FI 0.6; F11-2 1.9; Fl20 2.25; AI 0.9 ; CI 0.4; ICI 0.35; SDI 1.3; NI 2.7.

Colour. Yellow overall, including antennae and legs, with posterior half of T1-3 slightly darker; ovipositor sheath dark brown to black, pterostigma yellow, borderd posteriorly with black, wing veins dark brown to black, except sub-basal part of Rs $+2 \mathrm{r}$ which is yellow margined dorsally with black, wing sclerite light orange to yellow; mandibular teeth black. 
HEAD. Mandible short and stout, hardly twisted, upper tooth barely longer than lower tooth, bare, without groove on outer surface; clypeus superficially punctate medially, exceptionally convex in profile, ventral margin blunt and in-turned; face $1.2 \times$ as high as wide, smooth; gena relatively broad and slightly swollen behind eyes; occipital carina complete and thin; antenna long and slender, with 58 flagellomeres.

Mesosoma. Pronotum, mesoscutum, mesopleuron and metapleuron nearly smooth and shiny, with very slight fine rugosity especially ventrally; epicnemial carina distinct to level of ventral corner of pronotum; postpectal carina distinct and complete; notauli weak, but exceptionally long, extending over entire mesoscutum length; scutellum densely, finely punctate, carinate to near apex; basal transverse carina of propodeum sharply distinct; anterior area of propodeum smooth, posterior area finely transversely striate, concave postero-medially.

Wings. Disco-submarginal cell of fore wing with well developed fenestra; proximal sclerite commashaped and connected to very long distal sclerite extending to Rs $+2 \mathrm{r}$; central sclerite totally absent; $\mathrm{Rs}+2 \mathrm{r}$ more or less straight, sub-basally thickened; cu-a subopposite to Rs \& M; hind wing with 7 distal hamuli on R1.

LEGS. Fore tibia sparsely setose and with a crown of apical brownish spines; hind coxa in profile about $1.5 \times$ as long as high; hind tarsal claws symmetrical, with 8 subequal teeth, that are slightly shorter than claw length.

Metasoma. Stout, widened posteriorly; thyridium slender, extending to slightly more than basal third of T2; ovipositor hardly reaching beyond metasomal apex, with dark brown sheath.

\section{Male}

Unknown.

\section{BOLD Identification Number}

ADB3498.

\section{Remarks}

This species is morphologically exceptional within Enicospilus. The hardly twisted mandibles, strongly convex clypeus and very long notauli are characters which occur rarely in the genus. Their combination makes E. arabicus sp. nov. unique.

\section{Distribution}

Saudi Arabia.

Enicospilus bicoloratus Cameron, 1912

Figs 3B, 6B, 10B, 14B, 18B, 22B, 29B

E. bicoloratus Cameron, 1912: 388, ô.

Diagnosis (after Gauld \& Mitchell 1978)

B 19 ; F 12-15; ML 0.4; CT 1.4; OOL 0.1; POL 0.5; FI 0.5-0.6; Fl1-2 1.6-1.8; Fl20 2.3-2.6; AI 0.450.95; CI 0.35-0.6; ICI 0.4-0.6; SDI 1.2-1.4; NI 2.6.

Body yellowish brown to orange, orbits paler; mandible with upper tooth $1.1-1.5 \times$ as long as lower tooth; clypeus convex in profile, with ventral margin in-turned; face $1.2 \times$ as high as wide; antenna with 50-56 flagellomeres; mesopleuron and metapleuron finely densely punctate; basal transverse carina of propodeum distinct, anterior area of propodeum punctate or nearly so, posterior area coarsely 
striate; proximal sclerite triangular and moderately extended distally; central sclerite small, circular and uniformly sclerotized; hind wing with 5-7 distal hamuli on R1; fore tibia not spinose.

\section{Material examined}

SAUDI ARABIA: 1 q, Wadi Ghanuna (Al Baha), sweep net, 12 May 2011, leg. Fadl et al. (KSMA); 1 , , Raydah (Asir), light trap 9, 26 Aug. 2014, leg. Al Dhafer et al. (KSMA); 1 , , Raydah (Asir), light trap 5, 21 Oct. 2014, leg. Al Dhafer et al. (KSMA); 1 q, Raydah (Asir), light trap 9, 17 Nov. 2015, leg. Al Dhafer et al. (KSMA).

\section{Distribution}

Angola, Cameroon, Central African Republic, Democratic Republic of Congo, Ethiopia, Ivory Coast, Madagascar, Malawi, Nigeria, Sierra Leone, South Africa, Uganda (Gauld \& Mitchell 1978; Yu et al. 2012), Zimbabwe (Rousse \& van Noort 2014); Saudi Arabia (new record).

Enicospilus brevicornis (Masi, 1939)

Figs 3C, 6C, 10C, 14C, 18C, 22C, 29C

Amesospilus brevicornis Masi, 1939: 32, ․

\section{Diagnosis (after Gauld \& Mitchell 1978)}

B 13-17; F 11-14; ML 0.3; CT 1.4-1.5; OOL 0.1; POL 0.58; FI 0.5-0.55; Fl1-2 1.7-1.9; Fl20 1.2-1.6; AI 0.4-1.0; CI 0.15-0.25; ICI 0.5-0.7; SDI 1.1-1.2; NI 2.0.

Body reddish brown overall, with upper and ventral sides of metasoma (in profile) black starting from T3; yellowish white in the following parts: posterior orbits of eyes, very thin along orbits (except upper third); pterostigma and most of Rs $+2 \mathrm{r}$ light red in colour; mandible with upper tooth subequal to lower one; clypeus slightly convex in profile, with ventral margin slightly in-turned; face $1.3 \times$ as high as broad; antenna with 43-47 flagellomeres; mesopleuron and metapleuron puncto-striate and longitudinally striate; basal transverse carina of propodeum distinct, anterior area nearly smooth to finely transversely sculptured, posterior area concentrically striate; proximal sclerite triangular, fully sclerotized; central sclerite totally absent; hind wing with 7-8 distal hamuli on R1; fore tibia sparsely setose.

\section{Material examined}

SAUDI ARABIA: 10 q $q$, Raydah (Abha), sweep net, on olive, 26 Aug. 2014 (leg. Al Dhafer et al.); 3 우, same data but on Juniper (EFC); 2 우, Raydah (Abha), light trap 4, 27 Apr. 2014, leg. Al Dhafer et al. (KSMA); 2 우, Raydah (Abha), light trap 7, 6 Jun. 2014, leg. Al Dhafer et al. (KSMA); 1 , Raydah (Abha), light trap 8, 21 Feb. 2014, leg. Al Dhafer et al. (KSMA); 1 + , Raydah (Abha), light trap (House), 26 Apr. 2014, leg. Al Dhafer et al. (KSMA); 1 \&, Wadi Bisha (K. Mushayt), sweep net, 27 Apr. 2014, leg. Sharaf et al. (KSMA).

\section{Previous records from Saudi Arabia}

Asir (Gauld \& Mitchell 1978).

\section{BOLD Identification Number}

ADB3412.

\section{Distribution}

Egypt, Ethiopia, Saudi Arabia, South Africa (Gauld \& Mitchell 1978), Democratic Republic of Congo, Kenya, Madagascar, Sudan, Uganda, Yemen (Yu et al. 2012). 

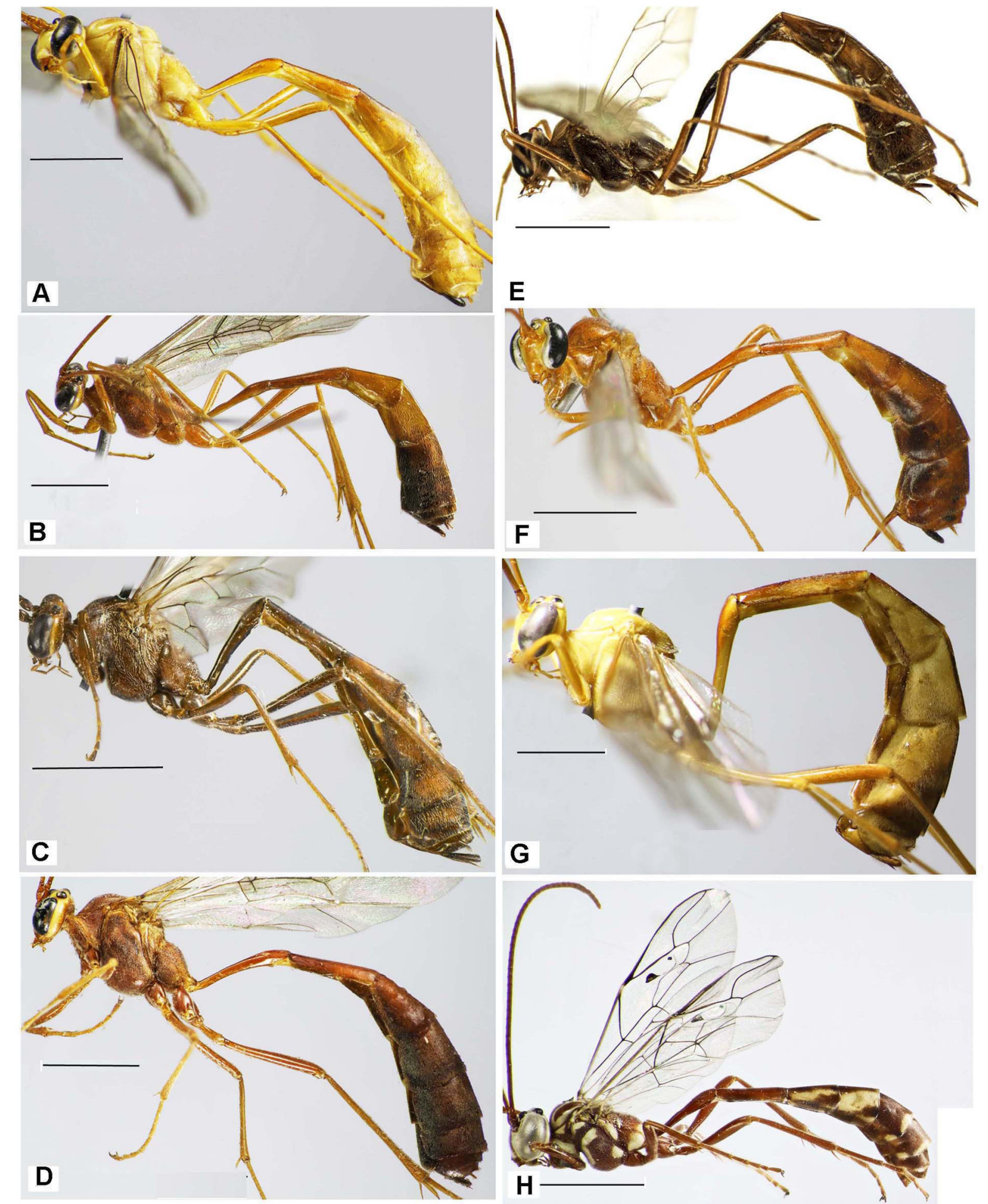

Fig. 3. Lateral habitus. A. Enicospilus arabicus Gadallah \& Soliman sp. nov. B. E. bicoloratus Cameron, 1912. C. E. brevicornis (Masi, 1939). D. E. capensis (Thunberg, 1822). E. E. divisus (Seyrig, 1935). F. E. dubius (Tosquinet, 1896). G. E. grandiflavus Townes \& Townes, 1973. H. E. mirabilis Soliman \& Gadallah sp. nov. Scale bars $=2.5 \mathrm{~mm}$. 


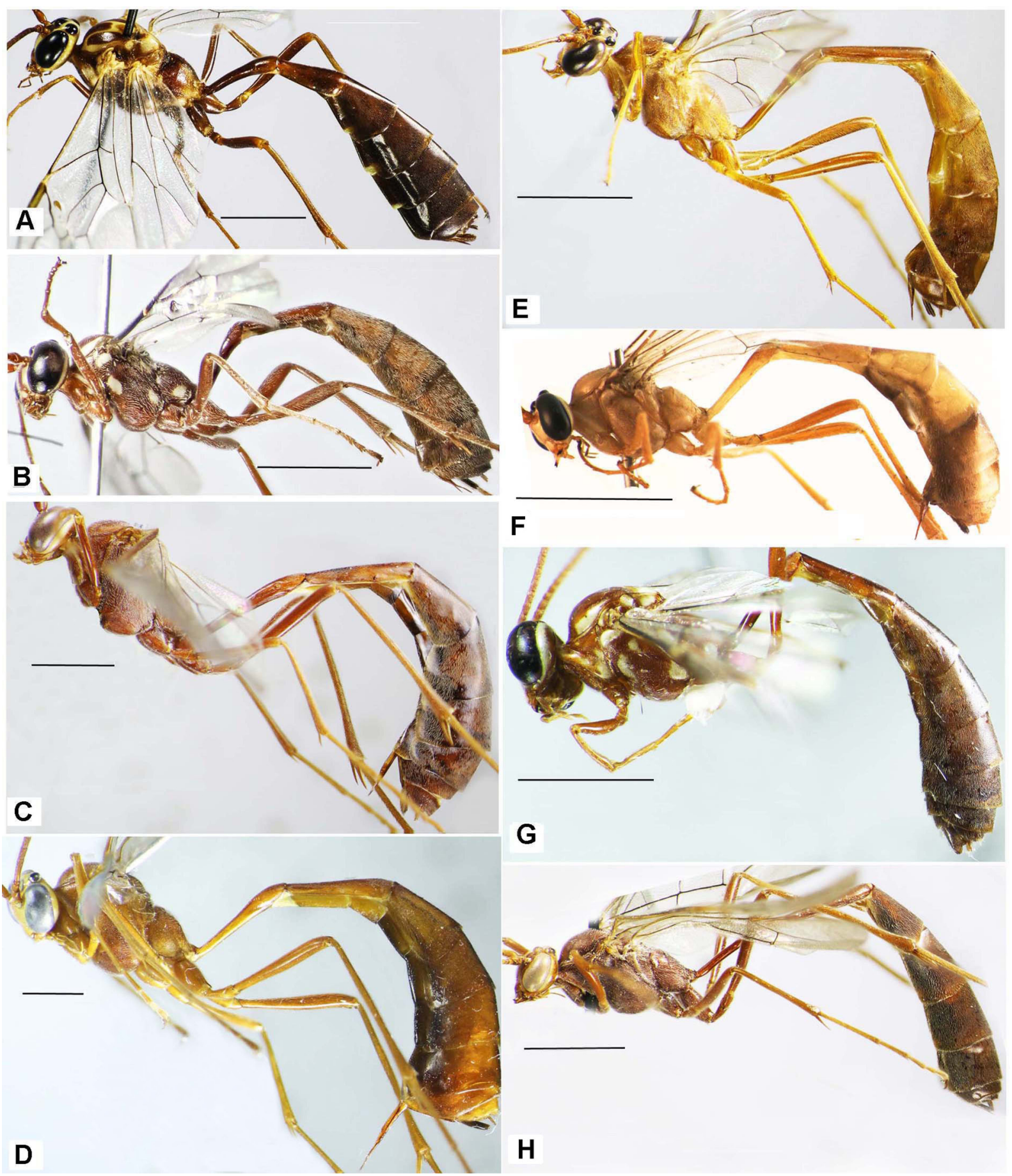

Fig. 4. Lateral habitus. A. Enicospilus nervellator Aubert, 1966. B. E. oculator Seyrig, 1935. C. E. odax Gauld \& Mitchell, 1978. D. E. oweni Gauld \& Mitchell, 1976. E. E. pacificus (Holmgren, 1868). F. E. pallidus (Taschenberg, 1875). G. E. pseudoculator Gadallah \& Soliman sp. nov. H. E. rundiensis Bischoff, 1915. Scale bars $=2.5 \mathrm{~mm}$. 

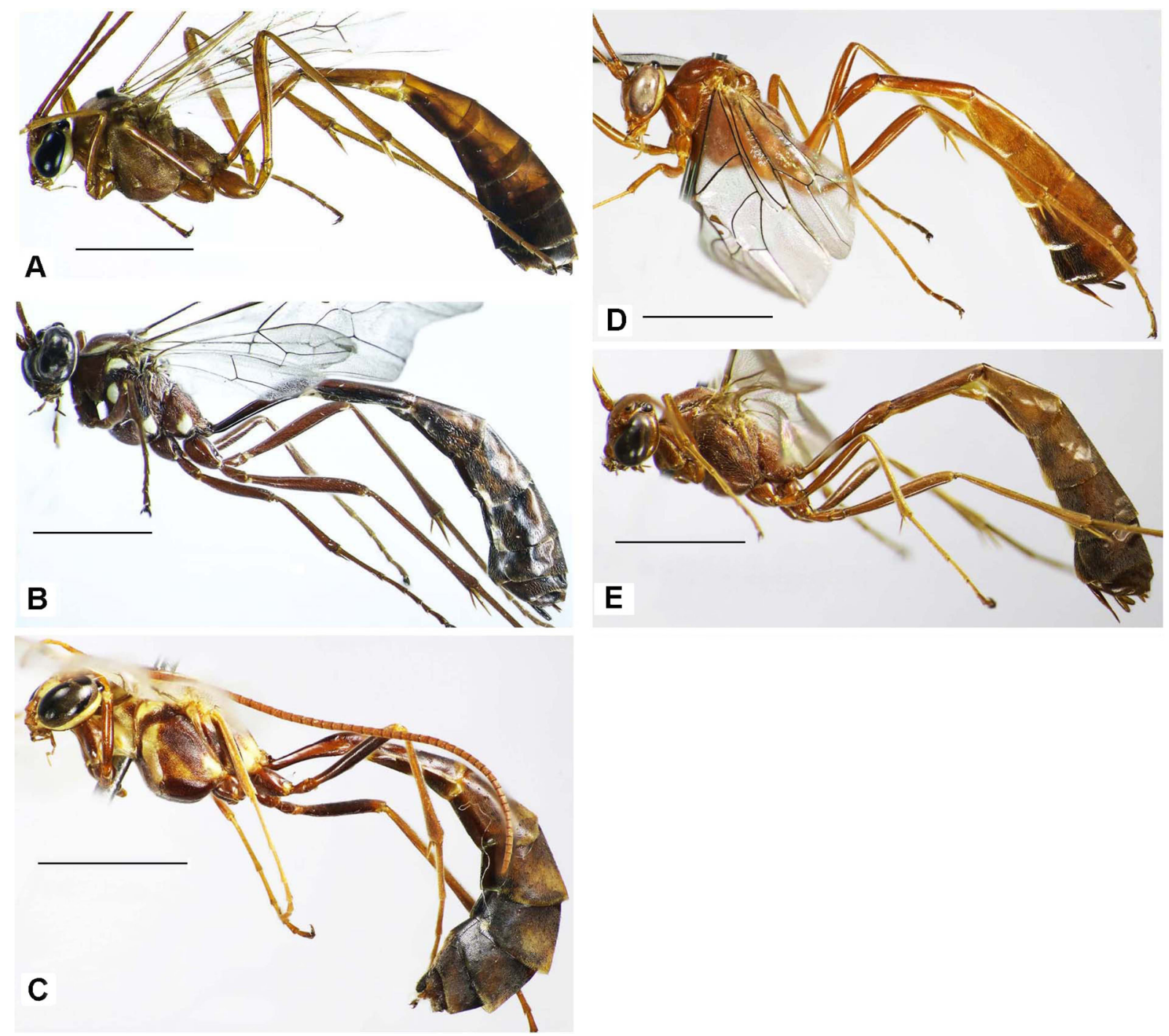

Fig. 5. Lateral habitus. A. Enicospilus senescens (Tosquinet, 1896). B. E. shadaensis Gadallah \& Soliman sp. nov. C. E. splendidus Rousse, Soliman \& Gadallah sp. nov. D. E. sp. 2 cf. bicoloratus Cameron, 1915. E. E. sp. 1. Scale bar $=2.5 \mathrm{~mm}$. 
Enicospilus capensis (Thunberg, 1822)

Figs 3D, 6D, 10D, 14D, 18D, 23A, 29D

Ichneumon capensis Thunberg, 1822: 314, ㅇ.

Ophion antancarus Saussure, 1892: 21.

Henicospilus praedator Enderlein, 1921: 28, ㅇ

Henicospilus incarinatus Enderlein, 1921: 30, §

Henicospilus euxoae Wilkinson, 1928: 261,, .

Enicospilus obnoxius Seyrig, 1935: 75, ㅇ.

Diagnosis (after Gauld \& Mitchell 1978)

B 16-19; F 11-15; ML 0.2-0.3; CT 1.7-2.2; OOL 0.2; POL 0.8; FI 0.47-0.5; Fl1-2 1.5-1.7; F120 1.5-2.0; AI 0.4-0.8; CI 0.35-0.5; ICI 0.43-0.6; SDI 1.28-1.5; NI 1.8 .

Body reddish brown overall, orbits and gena pale yellow; mandible slightly twisted, with inner groove along outer margin that is lined with a fringe of pale setae, lower tooth slightly more than $2.0 \times$ as long as upper tooth; clypeus convex in profile, with ventral margin in-turned ventrally; face subquadrate, 0.85-1.1 $\times$ as high as wide; antenna with 46-54 flagellomeres; mesopleuron and metapleuron closely and finely punctate; basal transverse carina of propodeum distinct, anterior area of propodeum nearly smooth to transversely finely shagreened, posterior area coarsely rugose; proximal sclerite dome-shaped; central scelrite small, not uniformly sclerotized; distal sclerite transverse comma-shaped; hind wing with 7 distal hamuli on R1; fore tibia sparsely setose.

\section{Material examined}

SAUDI ARABIA: 2 우, Shada Al Ala (Al Baha), sweep net, on Cactus, 17 Oct. 2014, leg. Al Dhafer et al. (EFC); 4 우, Shada Al Ala (Al Baha), light trap 4, 15 Feb. 2014, leg. Al Dhafer et al. (KSMA); 1 , Shada Al Ala (Al Baha), light trap 5, 15 Feb. 2014 , leg. Al Dhafer et al. (KSMA); 3 우, Shada Al Ala (Al Baha), light trap (House), 16 Feb. 2014, leg. Fadl H. (KSMA); 1 q, Raydah (Abha), light trap 4, 6 Jun. 2014, leg. Al Dhafer et al. (KSMA); 2 우, Deirab (Riyadh), light trap, 21-25 Oct. 1985, leg.? (KSMA); 4 우, Deirab (Riyadh), light trap, 4 Nov. 1985, leg.? (KSMA); 1 q, Deirab (Riyadh), light trap, 1 Dec. 1985, leg.? (KSMA); 1 O: Al Waseel (Riyadh), light trap, 8 Dec. 2012, leg. Sonbati S. (KSMA); 1 q, Al Kharg (Riyadh), light trap, 22 Feb. 2004 , leg. Al Oqeal Y. (KSMA); 1 +, Al Amaryiah (Riyadh), light trap, 28 Jan. 2008, leg. Boy Valenza D. (KSMA).

\section{Previous records from Saudi Arabia}

Asir (Gauld \& Mitchell 1978).

\section{BOLD Identification Number}

AAI5183.

\section{Distribution}

Burundi, Democratic Republic of Congo, Kenya, Lesotho, Mauritius, Rhodesia, Rwanda, Senegal, Sierra Leone, South Africa, Tanzania, Uganda, Yemen (Gauld \& Mitchell 1978), China, Ethiopia, Gabon, India, Indonesia, Japan, Malaysia, Philippines, Sri Lanka (Yu et al. 2012), Madagascar (Gauld \& Mitchell 1978; Madl 1996). 
Enicospilus divisus (Seyrig, 1935)

Figs 3E, 6E, 10E, 14E, 18E, 23B, 29E

Schizospilus divisus Seyrig, 1935: 80, ․ .

Diagnosis (after Gauld \& Mitchell 1978)

B19; F 15.5; ML 0.3; CT 1.4; OOL 0.1; POL 0.7; FI 1.0; Fl1-2 1.6; F120 2.0; AI 1.0; CI 0.5; ICI 0.3; SDI 1.4; NI 2.2 .

Dark reddish brown overall, with vertex, posterior margin of head, face (except a median narrow dark longitudinal band extending from fore ocellus to base of clypeus) and most of clypeus (except reddish ventral part) whitish; labrum reddish, mandible red (except black teeth) and slender, with upper tooth subequal to inner tooth; clypeus convex in profile, ventral margin in-turned; face $1.3 \times$ as high as wide; mesopleuron puncto-striate, metapleuron finely shagreened; basal transverse carina of propodeum distinct; propodeum finely shagreened anteriorly, finely wrinkled posteriorly; proximal sclerite triangular, fully sclerotized; central sclerite subdivided and U-shaped; hind wing with 7 distal hamuli on R1; fore tibia sparsely setose.

\section{Material examined}

SAUDI ARABIA: 1 q, Raydah (Asir), light trap 9, 30 Jan. 2015, leg. Al Dhafer et al. (KSMA).

\section{BOLD Identification Number}

ABD4430.

\section{Distribution}

Widely distributed throughout East Africa from Ethiopia to South Africa (Gauld \& Mitchell 1978), Uganda (Rousse \& van Noort 2014); Saudi Arabia (new record).

Enicospilus dubius (Tosquinet, 1896)

Figs 3F, 6F, 10F, 14F, 18F, 23C, 29F

Ophion (Enicospilus) dubius Tosquinet, 1896: 390, ㅇ.

Ophion (Enicospilus) anceps Tosquinet, 1896: 392, ठ̂.

Henicospilus angustatus Szépligeti, 1906: 136, ․

Amesospilus gulosus Seyrig, 1935: 60, ․

Diagnosis (after Gauld \& Mitchell 1978)

B17-19; F 11-16; ML 0.2-0.3; CT 1.4-1.6; OOL 0.2; POL 0.53; FI 0.6-0.65; Fl1-2 1.6-1.8; Fl20 2.5-3.0; AI 1.1-2.0; CI 0.5-0.7; ICI 0.25-0.45; SDI 1.29-1.5; NI 1.88.

Body including antennae and legs reddish brown, metasoma darker in colour, orbits paler; mandible with upper tooth 1.4-1.5 $\times$ as long as lower tooth; clypeus convex in profile, with ventral margin in-turned; face $1.3 \times$ as high as wide; antenna with 52-61 flagellomeres; mesopleuron impunctate, longitudinally striate, metapleuron finely transversely striate; basal transverse carina of propodeum distinct, anterior area nearly smooth or finely rugose, posterior area concentrically rugose; proximal sclerite triangular, fully sclerotized; central sclerite totally absent, distal sclerite small, crescent-shaped; hind wing with 6-8 distal hamuli on R1; fore tibia not spinose. 

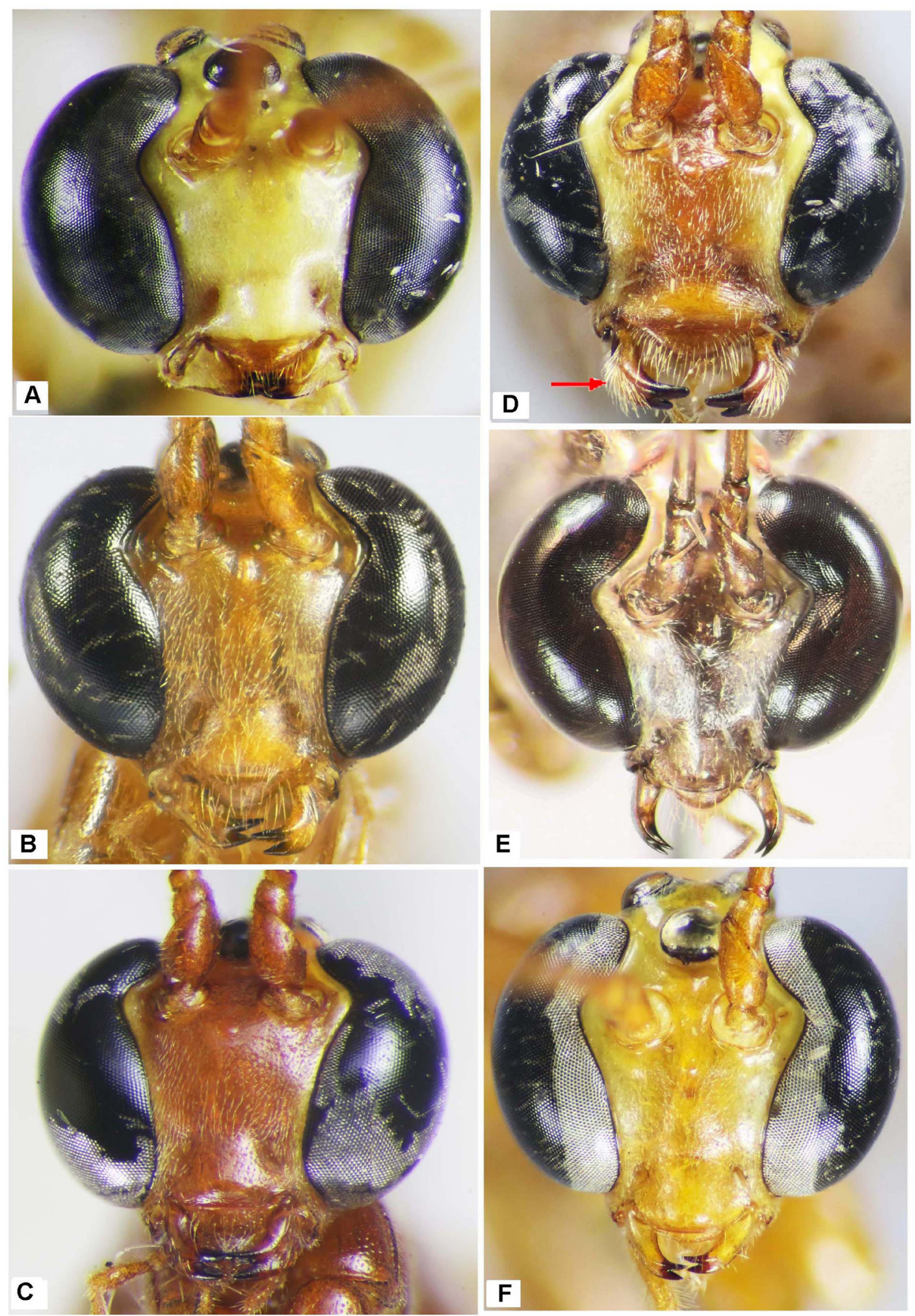

Fig. 6. Anterior view of head. A. Enicospilus arabicus Gadallah \& Soliman sp. nov. B. E. bicoloratus Cameron, 1912. C. E. brevicornis (Masi, 1939). D. E. capensis (Thunberg, 1822). E. E. divisus (Seyrig, 1935). F. E. dubius (Tosquinet, 1896). 

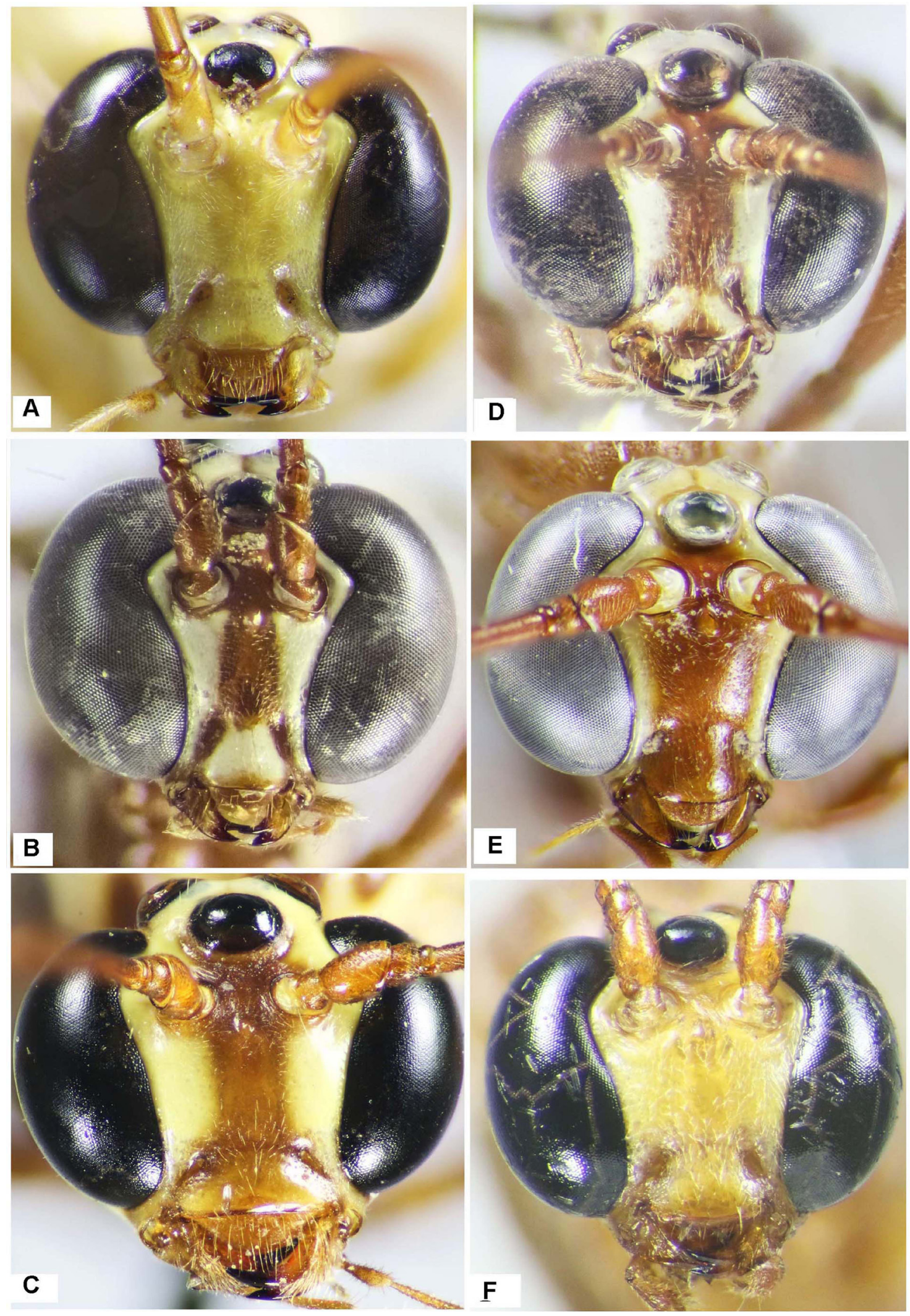

Fig. 7. Anterior view of head. A. Enicospilus grandiflavus Townes \& Townes, 1973. B. E. mirabilis Soliman \& Gadallah sp. nov. C. E. nervellator Aubert, 1966. D. E. oculator Seyrig, 1935. E. E. odax Gauld \& Mitchell, 1978. F. E. oweni Gauld \& Mitchell, 1978. 

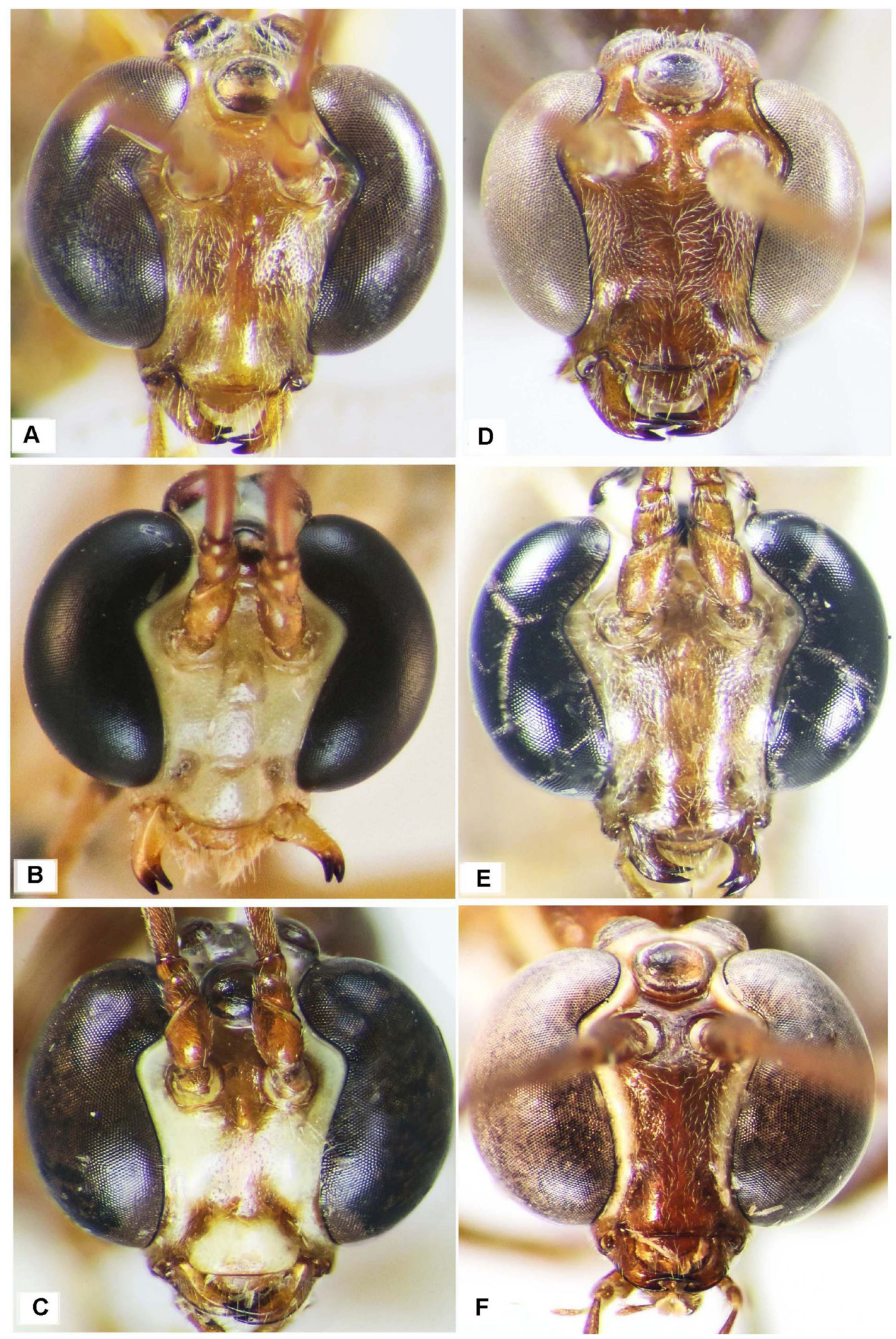

Fig. 8. Anterior view of head. A. Enicospilus pacificus (Holmgren, 1868). B. E. pallidus (Taschenberg, 1875). C. E. pseudoculator Gadallah \& Soliman sp. nov. D. E. rundiensis Bischoff, 1915. E. E. senescens (Tosquinet, 1896). F. E. shadaensis Gadallah \& Soliman sp. nov. 

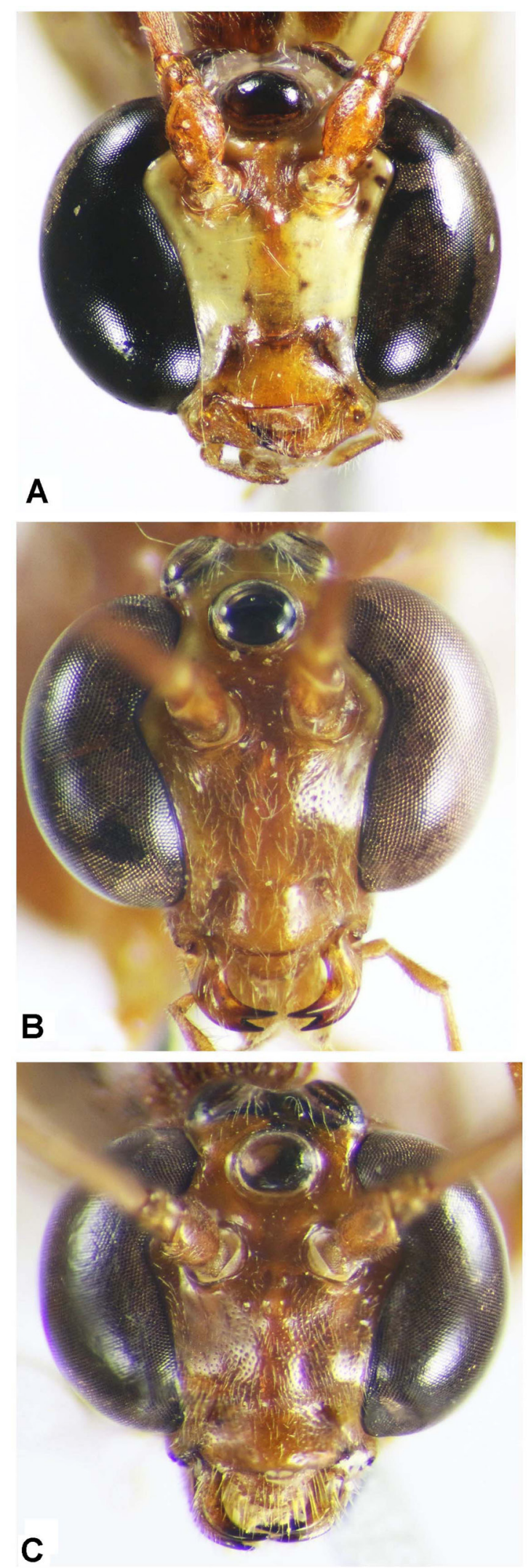

Fig. 9. Anterior view of head. A. Enicospilus splendidus Rousse, Soliman \& Gadallah sp. nov. B. Enicospilus sp. 2 cf. bicoloratus Cameron, 1915. C. Enicospilus. sp. 1 


\section{Material examined}

SAUDI ARABIA: 1 , Raydah (Asir), light trap (House), 27 Apr. 2014, leg. Al Dhafer et al. (EFC); 2 우, Raydah (Asir), light trap 7, 20 Feb. 2014, leg. Al Dhafer et al. (KSMA); 1 , , Raydah (Asir), light trap 1, 27 Apr. 2014, leg. Al Dhafer et al. (KSMA); 2 우, Raydah (Asir), light trap 4, 26-27 Apr. 2014 leg. Al Dhafer et al. (KSMA); 2 우, 1 ô, Raydah (Asir), light trap 3, 6 Jun. 2014, leg. Al Dhafer et al. (KSMA); 3 우, Raydah (Asir), light trap 4, 6 Jun. 2014, leg. Al Dhafer et al. (KSMA); 1 q, Raydah (Asir), light trap 5, 20 Oct. 2014, leg. Al Dhafer et al. (KSMA); 1 \&, Raydah (Asir), light trap 5, 18 Nov. 2015, leg. Al Dhafer et al. (KSMA); 1 +, Raydah (Asir), light trap 8, 18 Nov. 2015 , leg. Al Dhafer et al. (KSMA); 2 q 9 , Raydah (Asir), light trap 9, 18 Nov. 2015, leg. Al Dhafer et al. (KSMA).

\section{BOLD Identification Number}

ABD4296.

\section{Distribution}

Angola, Burundi, Cameroon, Central African Republic, Ethiopia, Ghana, Ivory Coast, Kenya, Nigeria, Rwanda, Sierra Leone, South Africa, Tanzania (Gauld \& Mitchell 1978; Yu et al. 2012), Madagascar (Gauld \& Mitchell 1978; Madl 1996; Yu et al. 2012), Réunion (Gauld \& Mitchell 1978; Rousse \& Villemant 2012; Yu et al. 2012); Saudi Arabia (new record).

Enicospilus grandiflavus Townes \& Townes, 1973

Figs 3G, 7A, 11A, 15A, 19A, 24A, 30A

Enicospilus grandiflavus Townes \& Townes, 1973: 177 [replacement name for grandis Morley]. Henicospilus grandis Morley, 1912: 41, §̂.

Diagnosis (after Gauld \& Mitchell 1978)

B 26-30; F 17-19; ML 0.3; CT 1.5-1.7; OOL 0.1; POL 0.5; FI 0.65-0.7; Fl1-2 1.6-2.1; Fl20 1.3-1.5; AI 0.65-0.9; ICI 0.85-0.9; CI 0.22; SDI 1.1-1.2; NI 1.4.

Body predominately light yellow, metasoma darkened dorsally and apically; pterostigma and sclerites light yellow, wing venation black, tip of mandible black; mandible slightly twisted, with upper tooth about $2.0 \times$ as long as lower tooth; clypeus flat in profile, with straight to truncate free margin; face subquadrate, 1.1-1.4 $\times$ as high as wide; antenna with 54-60 flagellomeres; mesopleuron closely punctate to puncto-striate, metapleuron coarsely transversely rugose; basal transverse carina of propodeum distinct, anterior area of propodeum finely rugose, posterior area coarsely and concentrically striate; proximal sclerite obtusely angled without distal extension, central sclerite totally absent; hind wing with distal 7-8 hamuli on R1; fore tibia sparsely spinose; claws with black, regular and equal-sized comb of teeth, slightly shorter than claw length, shortened near base.

\section{Material examined}

SAUDI ARABIA: 1 ○, Raydah (Asir), light trap, on Cactus, 11 Dec. 2014, leg.? (EFC); 1 q, Raydah (Asir), light trap (House), 5 Sep. 2015, leg. Al Dhafer et al. (EFC); 1 d, Shada Al Ala (Al Baha), light trap (House), 3 Jun. 2014, leg. Al Dhafer et al. (EFC); 1 , Shada Al Ala (Al Baha), light trap 4, 3 Jun. 2014, leg. Al Dhafer et al. (EFC); 1 o, 1 q, Raydah (Asir), light trap (House), 8 Nov. 2015, leg. A1 Dhafer et al. (EFC); 2 $\widehat{\jmath}$, Shada Al Ala (Al Baha), sweep net on Acacia, 15 Nov. 2014, leg. Al Dhafer et al. (KSMA); 1 o, Shada Al Ala (Al Baha), light trap 4, 21 Apr. 2014, leg. Al Dhafer et al. (KSMA);

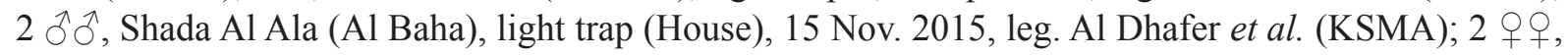
1 ऽ, Raydah (Asir), light trap 9, 17-18 Nov. 2015, leg. Al Dhafer et al. (KSMA). 


\section{BOLD Identification Number}

AAI5204.

\section{Distribution}

Madagascar, South EastAfrica(Gauld \& Mitchell 1978), Réunion, SouthAfrica (Rousse \& Villemant2012; Rousse \& van Noort 2014), Democratic Republic of Congo, Zimbabwe (Yu et al. 2012); Saudi Arabia (new record).

Enicospilus mirabilis Soliman \& Gadallah sp. nov. urn:1sid:zoobank.org:act:8BE2DB48-FE4D-4382-B327-2652AB99B259

Figs 3H, 7B, 11B, 15B, 19B, 24B, 30B

\section{Diagnosis}

Small and dark reddish brown overall including antennae and legs, with profuse white on all body regions, metasoma interspersed with ivory markings; mandible hardly twisted; clypeus flat in profile, ventral margin straight to truncate; face subquadrate, $1.1 \times$ as high as wide; antennae with 44 flagellomeres; mesoscutum with indistinct notauli; mesopleuron puncto-striate, metapleuron densely finely punctate; propodeum nearly smooth anteriorly and superficially foveolate posteriorly; proximal sclerite dome to triangular shaped, central sclerite small and circular, uniformly sclerotized.

\section{Etymology}

Latin adjective meaning "amazing", in reference to the wonderful appearance of this species.

\section{Type material}

\section{Holotype}

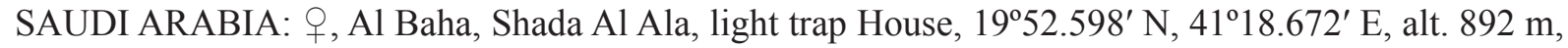
21 Apr. 2014, leg. Al Dhafer et al. (KSMA).

\section{Paratypes}

SAUDI ARABIA: 1 § , Raydah (Asir), light trap 6, on olive, 26 Aug. 2014, leg. Al Dhafer et al. (KSMA); 1 , Shada Al Ala (Al Baha), light trap (House), 26 Apr. 2014, leg. Al Dhafer et al. (KSMA); 1 , Raydah (Asir), light trap 7, 26 Apr. 2014, leg. Al Dhafer et al. (KSMA); 1 q, Raydah (Asir), light trap 6, 26 Aug. 2014, leg. Al Dhafer et al. (KSMA); 1 đ̃, Wadi Neera (Al Baha), light trap, 3 Mar. 2015, leg. Al Dhafer et al. (KSMA); 1 ${ }^{1}$, Shada Al Ala (Al Baha), light trap 4, 5 May 2015, leg. Al Dhafer et al. (KSMA); 1 , Shada Al Ala (Al Baha), light trap 4, 2 Sep. 2015, leg. Al Dhafer et al. (KSMA).

\section{Description}

Female (5 specimens)

Measurements. B 14; F 8; ML 0.5; CT 0.66; OOL 0.1; POL 1.13; FI 0.68; F11-2 1.6; Fl20 1.5; AI 0.57; CI 0.25; ICI 0.35; SDI 1.29; NI 2.1.

Colour. Reddish brown overall, including antennae and legs, with profuse white on the following: head (except a narrow middle area just between and beneath antennal bases to base of clypeus, as well as around clypeus and occiput), lateral border of mesoscutum as well as two very thin lines in middle, tegula, axilla, scutellum, anterior border of pronotum (interrupted medially), two large markings on lateral side of pronotum, subalar prominence, an elongate oval marking postero-laterally of propodeum, 
two large markings forming together an inverted L-shaped marking on upper side of mesopleuron and a small rounded one on its lower area just above base of mesocoxa, a transverse band at upper side of metapleuron and a small rounded marking at lower area just above metacoxa, metasoma with ivory markings laterally on T3-T7. Fore wing with yellowish pterostigma is bordered above and behind by black, wing veins black, sclerites black.

HEAD. Mandible hardly twisted, with slightly ridged and dark external margin and with two subequal to equal-sized teeth; labrum semicircular, about $1.3 \times$ as broad as long; clypeus flat in profile, ventral margin straight to truncate; face subquadrate, $1.1 \times$ as high as wide; gena moderately swollen behind eye; occipital carina complete and thin; antenna short, with 44 flagellomeres.

Mesosoma. Pronotum normal; mesoscutum nearly smooth and shiny; mesopleuron closely punctate, metapleuron puncto-striate; epicnemial carina distinct to level of ventral corner of pronotum; postpectal carina distinct and complete; notauli indistinct; scuto-scutellar groove strigated; scutellum smooth, carinate to apex; transverse basal carina of propodeum weak but distinct, propodeum nearly smooth to transversely shagreened, superficially wrinkled posteriorly.

WiNGS. Disco-submarginal cell of fore wing with well developed fenestra, proximal sclerite triangular to dome-shaped, central sclerite small circular and uniformly sclerotized; Rs $+2 \mathrm{r}$ very slightly thickened medially, more or less straight; hind wing with 4 distal hamuli on R1.

LEGS. Fore tibia not spinose.

Metasoma. Slender, T2 in profile $5 \times$ as long as high; thyridium ellipsoid, shallowly dilineated extending along anterior third of $\mathrm{T} 2$ laterally; ovipositor short, hardly extending beyond abdominal extremity.

Male (3 specimens)

Similar to female.

\section{BOLD Identification Number}

ADB4082.

\section{Remarks}

This species resembles E. oculator, but differs from it by the following combination of characters: in E. mirabilis sp. nov. clypeus almost ivory (except laterally and ventrally) (in E. oculator clypeus ivory only ventrally); propodeum and metapleuron each with a large oval ivory marking (in oculator no ivory markings on metapleuron and propodeum), metasoma with ivory markings (in oculator metasoma without ivory markings); mandibular teeth subequal (in oculator upper tooth $1.5 \times$ as long as lower tooth); posterior area of propodeum superficially or shallowly finely wrinkled (in oculator coarsely striate); central sclerite uniformly sclerotized (in oculator central sclerite weakly sclerotized proximally); F120 < 1.7 (in oculator F120 > 1.7); hind wing with 4 distal hamuli on R1 (in oculator with 5 distal hamuli on R1).

\section{Distribution}

Saudi Arabia. 
Enicospilus nervellator Aubert, 1966

Figs 4A, 7C, 11C, 15C, 19C, 24C, 30C, 33A

Enicospilus nervellator Aubert, 1966: 43, ․

Diagnosis (after Gauld \& Mitchell 1978)

B 17-18; F 11-15; ML 0.1-0.25; CT 1.7-2.1; OOL 0.12, POL 0.6; FI 0.5; Fl1-2 1.7-1.9; Fl20 1.2-1.5; AI 0.54-0.65; ICI 0.6-0.8; CI 0.3-0.4; SDI 1.38-1.5.

Body reddish brown to ferruginous overall, $\mathrm{T} 1, \mathrm{~T} 2$ and most of $\mathrm{T} 3$ bright red, the following parts pale yellow: face (except red middle, extending from fore ocellus to base of clypeus), posterior margin of head broadly, vertex, almost all pronotum, a middle U-shaped area on mesoscutum extending to its posterior margin as well as lateral margin, posterior half of propodeum, tegula, scutellum (except basally), subalar prominence, upper and lower areas of mesopleuron, lower half of metapleuron; mandible with upper tooth $2.0 \times$ as long as lower tooth; clypeus flat in profile, with ventral margin straight to truncate; face subquadrate, 1.1-1.25 $\times$ as high as wide; antenna with 50-52 flagellomeres; mesopleuron punctate, metapleuron shallowly puncto-striate; basal transverse carina of propodeum very weak but complete, anterior area of propodeum sparsely punctate, posterior area superficially rugose; proximal sclerite yellow (weakly sclerotized) dome-shaped, central sclerite longitudinal and weakly sclerotized; hind wing with 6-7 distal hamuli on R1; fore tibia considerably spinose; outer mid (and sometimes hind) tibial spur distinctly less than $0.4 \times$ as long as inner spur.

\section{Material examined}

SAUDI ARABIA: 1 \%, Rhodet Khorim (Riyadh), light trap (B), 6 Mar. 2012, leg. Al Dhafer et al. (EFC); 1 q, Ummul Hammam (Riyadh), light trap, 10 Feb. 2010, leg. Abdel-Azeem M. (KSMA); 1 ;, Al Khararah (Riyadh), light trap, 22 Feb. 2012, leg. Al Gharabawy et al. (KSMA); 1 +, Al Khararah (Riyadh), light trap, 7 Mar. 2012, leg. Al Dyrahim et al. (KSMA); 1 q, Rawdet Farshet Sheaal (Riyadh), light trap 1, 26 Jan. 2016, leg. Al Gharabawy et al. (KSMA); 3 우요, Rawdet Farshet Sheaal (Riyadh), light trap 10, 26 Jan. 2016, leg. Al Gharabawy et al. (KSMA); 1 , , Rawdet Farshet Sheaal (Riyadh), light trap 11, 26 Jan. 2016, leg. Al Gharabawy et al. (KSMA); 1 \%, Wadi Ghaihab (Riyadh), light trap, 27 Jan. 2016, leg. Al Gharabawy et al. (KSMA); 19 우, Rawdet Al Harmalyiah (Riyadh), light trap 1, 2 Feb. 2016, leg. Al Dhafer et al. (KSMA); 49 $\widehat{\jmath}, 104$ 우, Rawdet Al Harmalyiah (Riyadh), light trap 3, 2 Feb. 2016, leg. Al Dhafer et al. (KSMA); 1 ภ, 53 우, Rawdet Al Harmalyiah (Riyadh), light trap 5, 2 Feb. 2016, leg. Al Dhafer et al. (KSMA); 1 \%, Rawdet Al Harmalyiah (Riyadh), Malaise trap 4, 2 Feb. 2016, leg. Al Dhafer et al. (KSMA); 1 ㅇ, Wadi Hanifa (Riyadh), light trap, 4 Feb. 2016, leg. Al Dhafer (KSMA); 1 q, Wadi Sabean (Asir), light trap, 22 Feb. 2016, leg. Fadl et al. (KSMA).

\section{Previous records from Saudi Arabia}

Jeddah, Najd, Rub'al Khali (Gauld \& Mitchell 1978).

\section{BOLD Identification Number}

ADB4509.

\section{Distribution}

Algeria, Saudi Arabia (Gauld \& Mitchell 1978; Yu et al. 2012), Egypt (Shaumar 1966; Yu et al. 2012). 

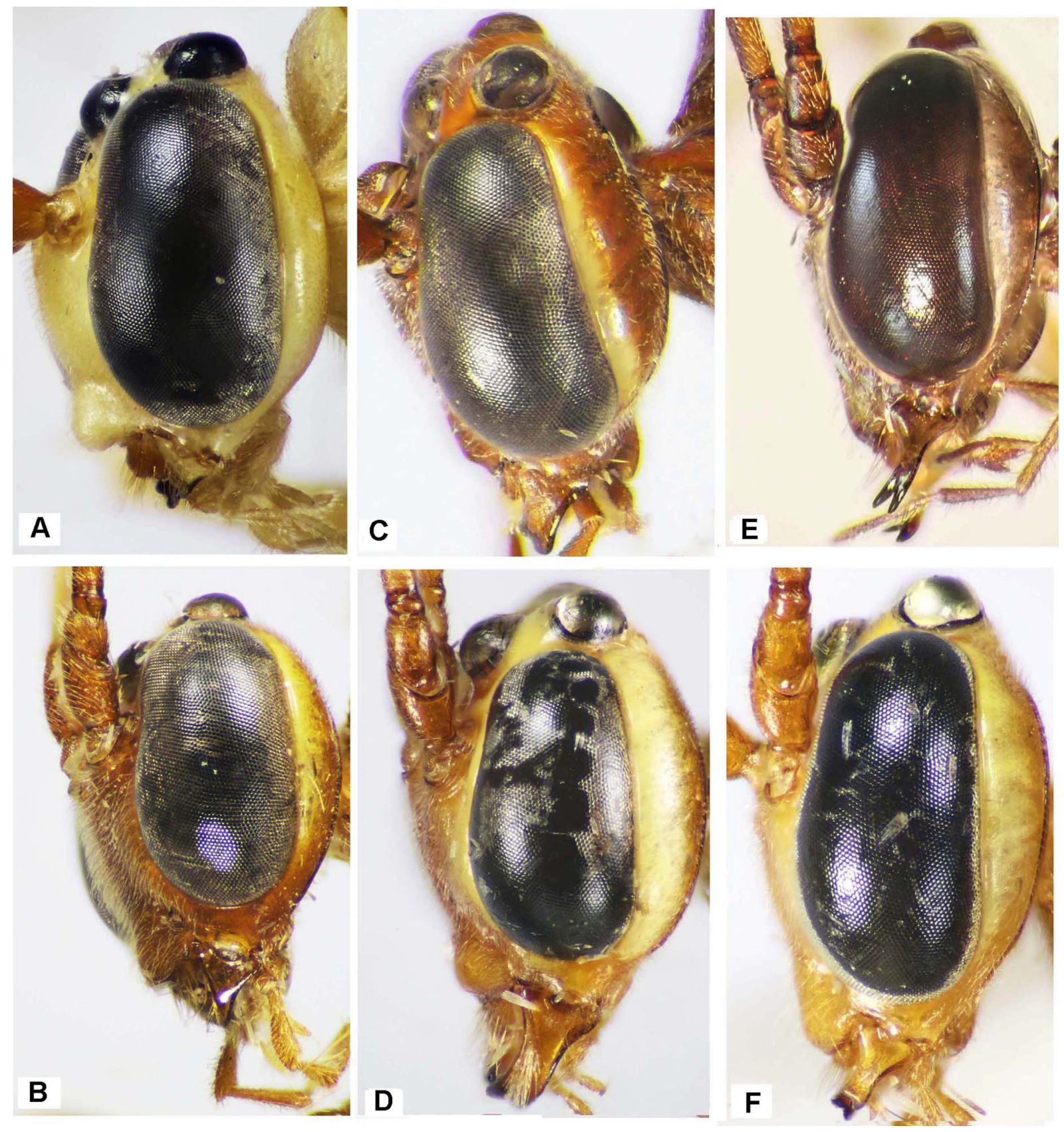

Fig. 10. Lateral view of head. A. Enicospilus arabicus Gadallah \& Soliman sp. nov. B. E. bicoloratus Cameron, 1912. C. E. brevicornis (Masi, 1939). D. E. capensis (Thunberg, 1822). E. E. divisus (Seyrig, 1935). F. E. dubius (Tosquinet, 1896). 

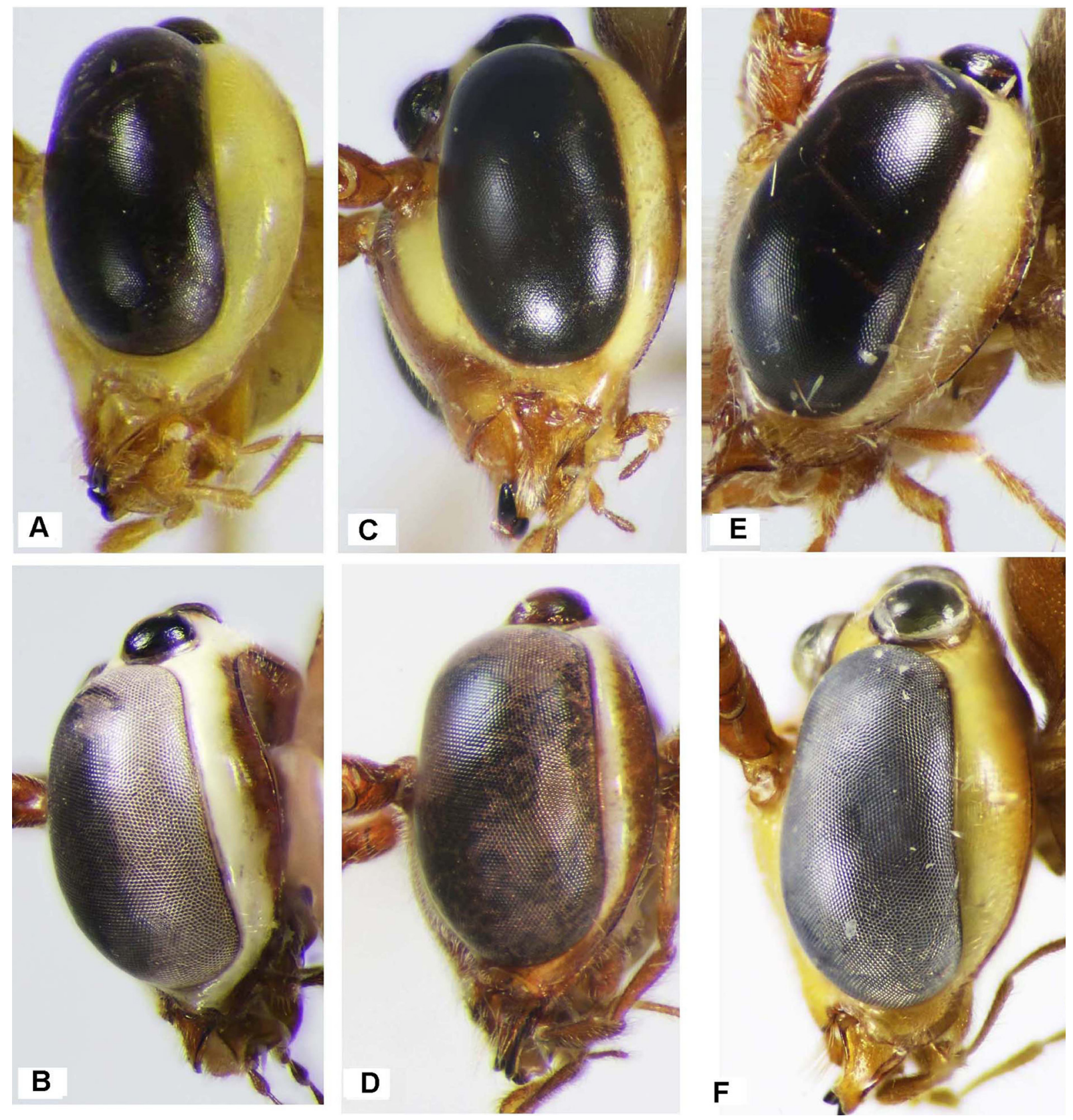

Fig. 11. Lateral view of head. A. Enicospilus grandiflavus Townes \& Townes, 1973. B. E. mirabilis Soliman \& Gadallah sp. nov. C. E. nervellator Aubert, 1966. D. E. oculator Seyrig, 1935. E. E. odax Gauld \& Mitchell, 1978. F. E. oweni Gauld \& Mitchell, 1978. 

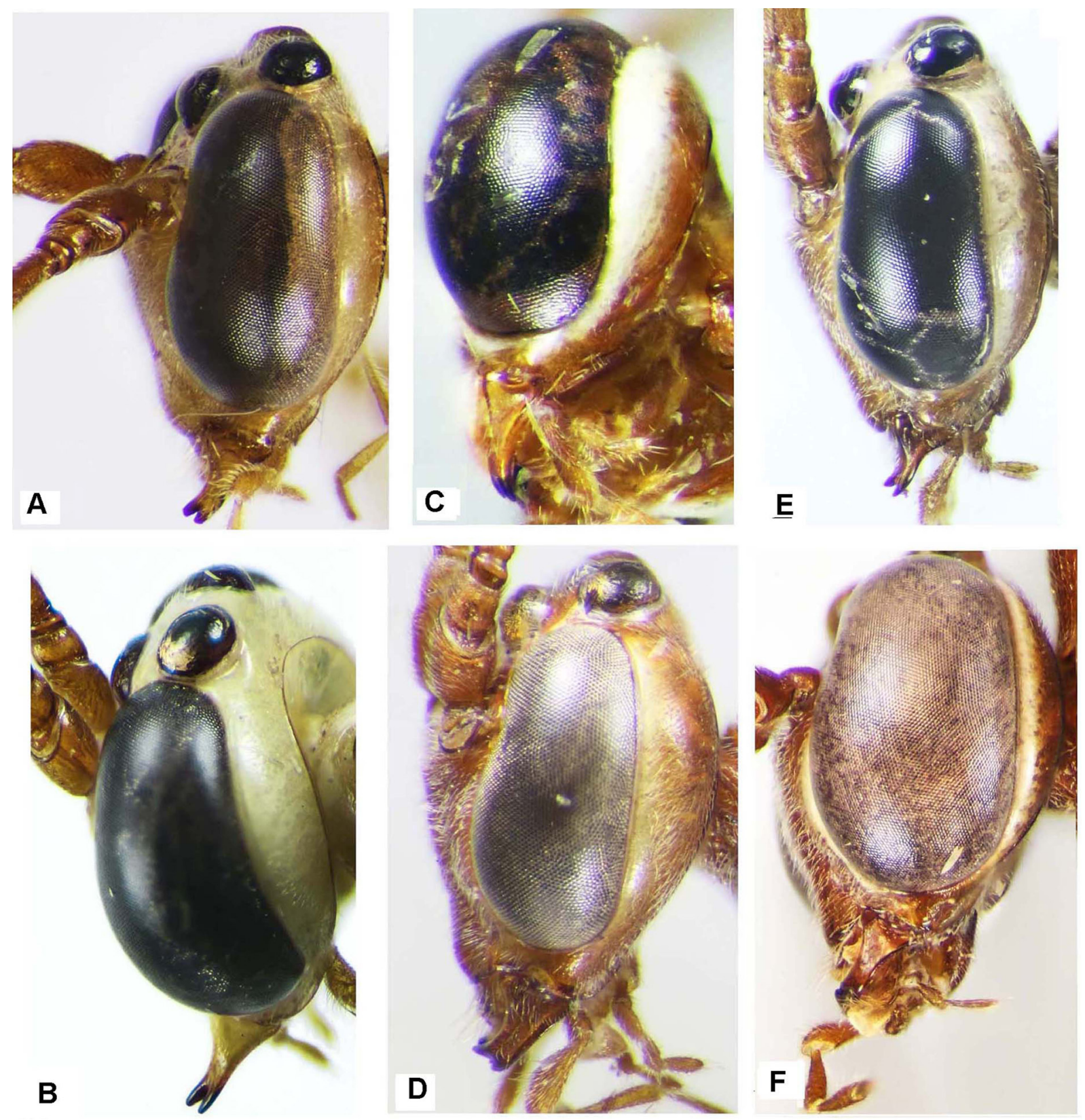

Fig. 12. Lateral view of head. A. Enicospilus pacificus (Holmgren, 1868). B. E. pallidus (Taschenberg, 1875). C. E. pseudoculator Gadallah \& Soliman sp. nov. D. E. rundiensis Bischoff, 1915. E. E. senescens (Tosquinet, 1896). F. E. shadaensis Gadallah \& Soliman sp. nov. 
Enicospilus oculator Seyrig, 1935

Figs. 4B, 7D, 11D, 15D, 19D, 25A, 30D

Enicospilus oculator Seyrig, 1935: 76,, .

Diagnosis (after Gauld \& Mitchell 1978)

B 15-16; F 8-10; ML 0.2; CT 1.7-1.8; OOL 0.1, POL 0.9; FI 0.8-0.92; Fl1-2 1.6-1.8; Fl20 1.8-2.1; AI 0.7-0.86; ICI 0.4-0.58; CI 0.2-0.25; SDI 1.1-1.3; NI 2.9.

Body entirely reddish brown including antennae and legs, with $\mathrm{T} 1$ mostly dark to black (except apically), with the following ivory parts: vertex, gena, face (except antennal toruli, middle area of face extending from fore ocellus to base of clypeus), clypeus (except basally, laterally and ventral margin), pronotum (patterned with red medially and laterally), lateral margins of mesoscutum, scutellum (except small reddish area basally), postscutellum, subalar prominences, large, oval shaped markings on anterior and posterior areas of mesopleuron, a large rounded to subquadrate marking on posterior area of metapleuron just above hind coxa, T1 black on basal half; mandible with lower tooth $1.6 \times$ as long as lower tooth; clypeus flat in profile, with ventral margin straight to truncate; face subquadrate, $1.0-1.1 \times$ as high as wide; antenna short, with 44-46 flagellomeres; mesopleuron and metapleuron finely and closely punctate, slightly coarser on mesopleuron; basal transverse carina weakly developed, but complete; anterior area of propodeum shallowly striate, posterior area finely transversely striate; proximal sclerite dark to black roundly triangular, central sclerite very small and circular, weakly sclerotized proximally; hind wing with 5 distal hamuli on R1; fore tibia sparsely spinose.

\section{Material examined}

SAUDI ARABIA: 1 + , Shada Al Ala (Al Baha), light trap 4, 8 Dec. 2014, leg. Al Dhafer et al. (EFC); 1 , Shada Al Ala (Al Baha), light trap (House), 13 Nov. 2015, leg. Al Dhafer et al. (KSMA); 1 , Shada Al Ala (Al Baha), light trap 6, 14 Nov. 2015, leg. Al Dhafer et al. (KSMA); 1 , Shada Al Ala (Al Baha), light trap 5, 14 Nov. 2015, leg. Al Dhafer et al. (KSMA).
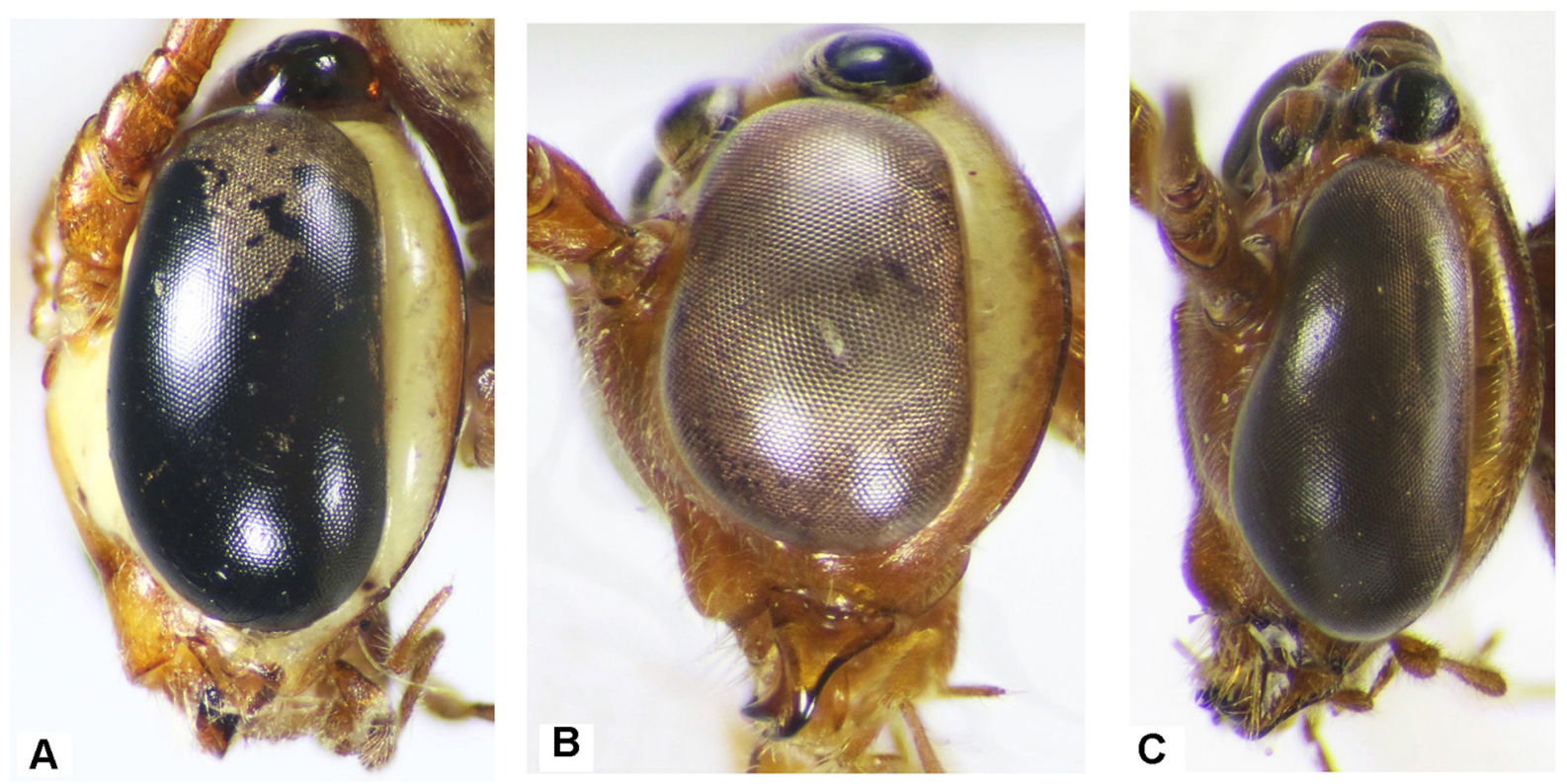

Fig. 13. Lateral view of head. A. Enicospilus splendidus Rousse, Soliman \& Gadallah sp. nov. B. Enicospilus sp. 2 cf. bicoloratus Cameron, 1915. C. Enicospilus sp. 1. 


\section{Previous records from Saudi Arabia}

Wadi Shuqub (Horstmann 1981).

\section{BOLD Identification Number}

ADB4114.

\section{Distribution}

Kenya, South Africa (Gauld \& Mitchell 1978; Yu et al. 2012), Saudi Arabia (Horstmann 1981; Yu et al. 2012), Zimbabwe (Rousse \& van Noort 2014).

Enicospilus odax Gauld \& Mitchell, 1978

Figs 4C, 7E, 11E, 15E, 19E, 25B, 30E

Enicospilus odax Gauld \& Mitchell, 1978: 137, ‥

Diagnosis (after Gauld \& Mitchell 1978)

B 16-30; F 14-20; ML 0.2; CT 1.3; OOL 0.04, POL 0.6; FI 0.6-0.66; Fl1-2 1.5-1.6; Fl20 1.6-2.0; AI 0.75; ICI 0.47-0.5; CI 0.28-0.36; SDI 1.4-1.45; NI 2.8 .

Relatively large-sized insects, body including antennae and legs reddish brown, vertex and orbits pale yellow to whitish; mandible with upper and lower teeth subequal; clypeus flat to hardly convex in profile, with ventral margin truncate; face 1.2-1.5 $\times$ as high as wide; antenna with 56-58 flagellomeres; mesopleuron closely punctate, metapleuron coarsely striate; posterior transverse carina of propodeum well distinct, anterior area finely and shallowly striate, posterior area coarsely and concentrically striate; fore wing with proximal sclerite acutely arrow-shaped, central sclerite weakly sclerotized; hind wing with 7-8 distal hamuli on R1; fore tibia sparsely spinose.

\section{Material examined}

SAUDI ARABIA: 5 우, 1 万, Raydah (Asir), light trap (House), 26 Aug. 2014, leg. Al Dhafer et al.; 1 specimen (without abdomen), Raydah (Asir), light trap (House), 26 Aug. 2014, leg. Al Dhafer et al.; 2 우, Shada Al Ala (Al Baha), light trap (House), 17 Oct. 2014, leg. Al Dhafer et al. (EFC); 1 ô, 1 , Wadi Yabah (Asir), 11 Oct. 2013, light trap, leg. Sonbati et al., (KSMA); 1 q, Raydah (Asir), light trap 6, 21 Feb. 2014, leg. Al Dhafer et al. (KSMA); 1 \%, Raydah (Asir), light trap 7, 21 Feb. 2014, leg. Al Dhafer et al. (KSMA); 1 + , Raydah (Asir), light trap 8, 21 Feb. 2014, leg. Al Dhafer et al. (KSMA); 3 우우, Raydah (Asir), light trap 9, 21 Feb. 2014 (leg. Al Dhafer et al.) (KSMA); 1 ㅇ, Tamniah Dam (Asir), light trap, 24 Feb. 2014, leg. Al Dhafer et al. (KSMA); 1 ㅇ, Wadi Rida (Asir), light trap, 24 Feb. 2014, leg. Al Dhafer et al. (KSMA); 3 o 9 , Raydah (Asir), light trap 4, 26 Apr. 2014, leg. Al Dhafer et al. (KSMA); 1 ㅇ, Raydah (Asir), light trap 5, 26 Apr. 2014, leg. Al Dhafer et al. (KSMA); 1 ㅇ, Raydah (Asir), light trap 6, 26 Apr. 2014, leg. Al Dhafer et al. (KSMA); 1 , , Raydah (Asir), light trap 7, 26 Apr. 2014, leg. Al Dhafer et al. (KSMA); 3 우, Raydah (Asir), light trap 9, 26 Apr. 2014, leg. Al Dhafer et al. (KSMA); 1 ô, 1 क, Shada Al Ala (Al Baha), light trap 1, 3 Jun. 2014, leg. Al Dhafer et al. (KSMA); 1 , , Raydah (Asir), light trap 2, 6 Jun. 2014, leg. Al Dhafer et al. (KSMA); 1 q, Raydah (Asir), light trap 3, 6 Jun. 2014, leg. Al Dhafer et al. (KSMA); 1 ô, 1 \%, Raydah (Asir), light trap 4, 6 Jun. 2014, leg. Al Dhafer et al. (KSMA); 1 +, Raydah (Asir), light trap 7, 6 Jun. 2014, leg. Al Dhafer et al. (KSMA); 3 우, Raydah (Asir), light trap 6, 26 Aug. 2014, leg. Al Dhafer et al. (KSMA); 1 , 1 Oे, Raydah (Asir), light trap 7, 20 Oct. 2014, leg. Al Dhafer et al. (KSMA); 3 우, Raydah (Asir), light 
trap 8, 20 Oct. 2014, leg. Al Dhafer et al. (KSMA); 1 q, Raydah (Asir), light trap 9, 20 Oct. 2014, leg. Al Dhafer et al. (KSMA); 1 ô, Raydah (Asir), light trap 6, 21 Oct. 2014, leg. Al Dhafer et al. (KSMA); 1 ㅇ, Shada Al Ala (Al Baha), light trap 2, 8 Dec. 2014, leg. Al Dhafer et al. (KSMA); 1 , Shada Al Ala (Al Baha), light trap 3, 8 Dec. 2014, leg. Al Dhafer et al. (KSMA); 1 + , Raydah (Asir), light trap 4, 11 Dec. 2014, leg. Al Dhafer et al. (KSMA); 1 क, Raydah (Asir), light trap 7, 11 Dec. 2014, leg. Al Dhafer et al. (KSMA); 1 , , Raydah (Asir), light trap 8, 11 Dec. 2014, leg. Al Dhafer et al. (KSMA); 1 q, Shada Al Ala (Al Baha), light trap 1, 27 Jan. 2015, leg. Al Dhafer et al. (KSMA); 1 , , Shada Al Ala (Al Baha), light trap 5, 27 Jan. 2015, leg. Al Dhafer et al. (KSMA); 2 o $q$, Raydah (Asir), light trap 6, 30 Jan. 2015, leg. Al Dhafer et al. (KSMA); 1 đ, Raydah (Asir), light trap 9, 5 Mar. 2015, leg. Al Dhafer et al. (KSMA); 1 \&, Raydah (Asir), Malaise trap 5, 8 May 2015, leg. Al Dhafer et al. (KSMA); 1 q, Raydah (Asir), light trap 7, 31 Jul. 2015, leg. Al Dhafer et al. (KSMA); 1 +, Raydah (Asir), light trap 7, 5 Sep. 2015, leg. Al Dhafer et al. (KSMA); 1 q, Raydah (Asir), light trap 8, 5 Sep. 2015, leg. Al Dhafer et al. (KSMA); 1 क, Raydah (Asir), light trap 6, 18 Nov. 2015, leg. Al Dhafer et al. (KSMA); 1 , Raydah (Asir), light trap 7, 18 Nov. 2015, leg. Al Dhafer et al. (KSMA); 1 +, Raydah (Asir), light trap 8, 18 Nov. 2015, leg. Al Dhafer et al. (KSMA); 2 우, Raydah (Asir), light trap 9, 18 Nov. 2015, leg. Al Dhafer et al. (KSMA).

\section{Description}

Male (7 specimens)

CoLour. Resembles female but with B 28 and F 20 totally reddish brown, with vertex, orbits and posterior margin of head pale yellowish, pronotum anteriorly light red, T3-7 of metasoma black laterally.

HEAD. Mandibles moderately twisted, with two subequal teeth; labrum $0.3 \times$ as high as broad; malar space $0.2 \times$ mandibular base; clypeus nearly smooth, flat to hardly convex in profile as in female, with ventral margin truncate; face finely shallowly punctate; gena slightly constricted behind eyes; lateral ocellus not touching eye; occipital carina complete; antenna with 54 flagellomeres, Fl1-2 1.25.

Mesosoma. Pronotum short, with transverse furrow; mesoscutum finely coriaceous, notauli indistinct; mesopleuron and metapleuron densely setose, but appears as if puncto-striate; basal carina of propodeum strong and complete, anterior area of propodeum finely coriaceous, posterior area coarsely and concentrically striate; postpectal carina complete.

FORE WING. Rs $+2 \mathrm{r}$ distinctly thickened medially, straight, fenestra less than length of 3r-m from Rs base; wing sclerites as in female (see diagnosis); hind wing with 1A straight basally, with 9 distal hamuli on R1. Fore tibia sparsely spinose.

Metasoma. Long and slender, S2 ending well before spiracle of T2; thyridium ellipsoid, very shallow and hardly visible.

\section{BOLD Identification Number}

ADB4115.

\section{Distribution}

Yemen (Gauld \& Mitchell 1978; Yu et al. 2012); Saudi Arabia (new record). 

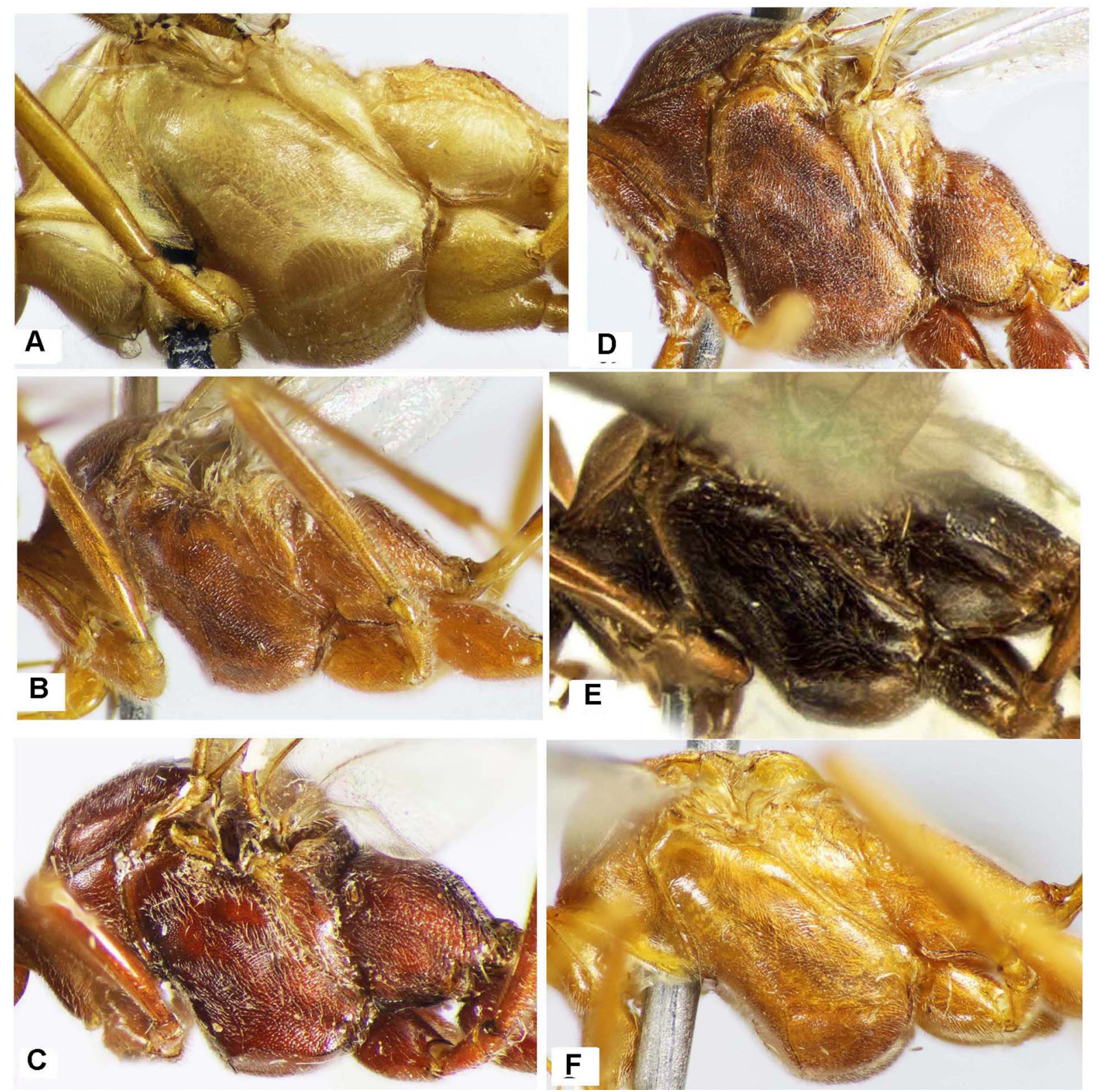

Fig. 14. Lateral aspect of mesosoma. A. Enicospilus arabicus Gadallah \& Soliman sp. nov. B. E. bicoloratus Cameron, 1912. C. E. brevicornis (Masi, 1939). D. E. capensis (Thunberg, 1822). E. E. divisus (Seyrig, 1935). F. E. dubius (Tosquinet, 1896). 

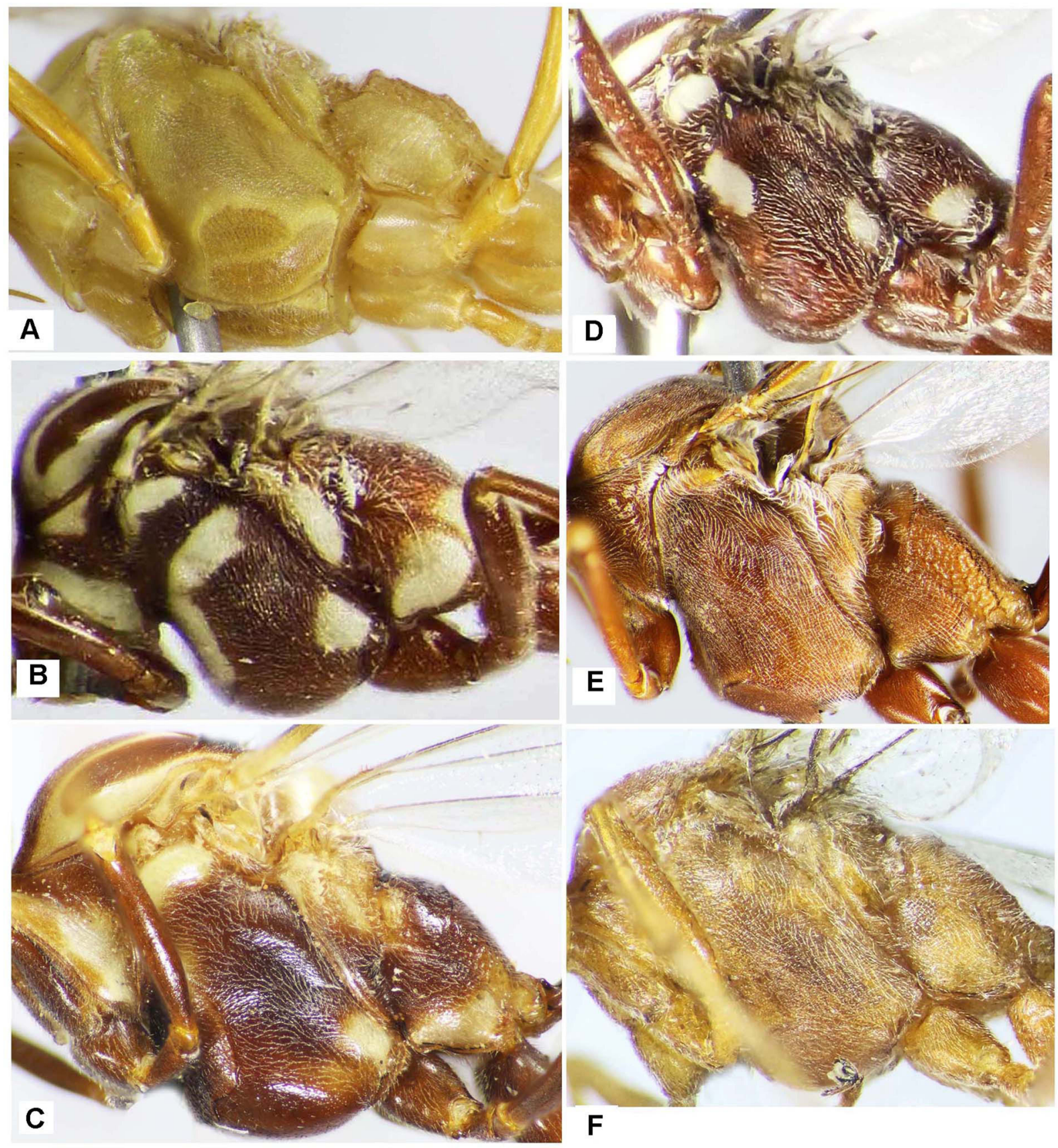

Fig. 15. Lateral aspect of meososoma. A. Enicospilus grandiflavus Townes \& Townes, 1973. B. E. mirabilis Soliman \& Gadallah sp. nov. C. E. nervellator Aubert, 1966. D. E. oculator Seyrig, 1935. E. E. odax Gauld \& Mitchell, 1978. F. E. oweni Gauld \& Mitchell, 1978. 

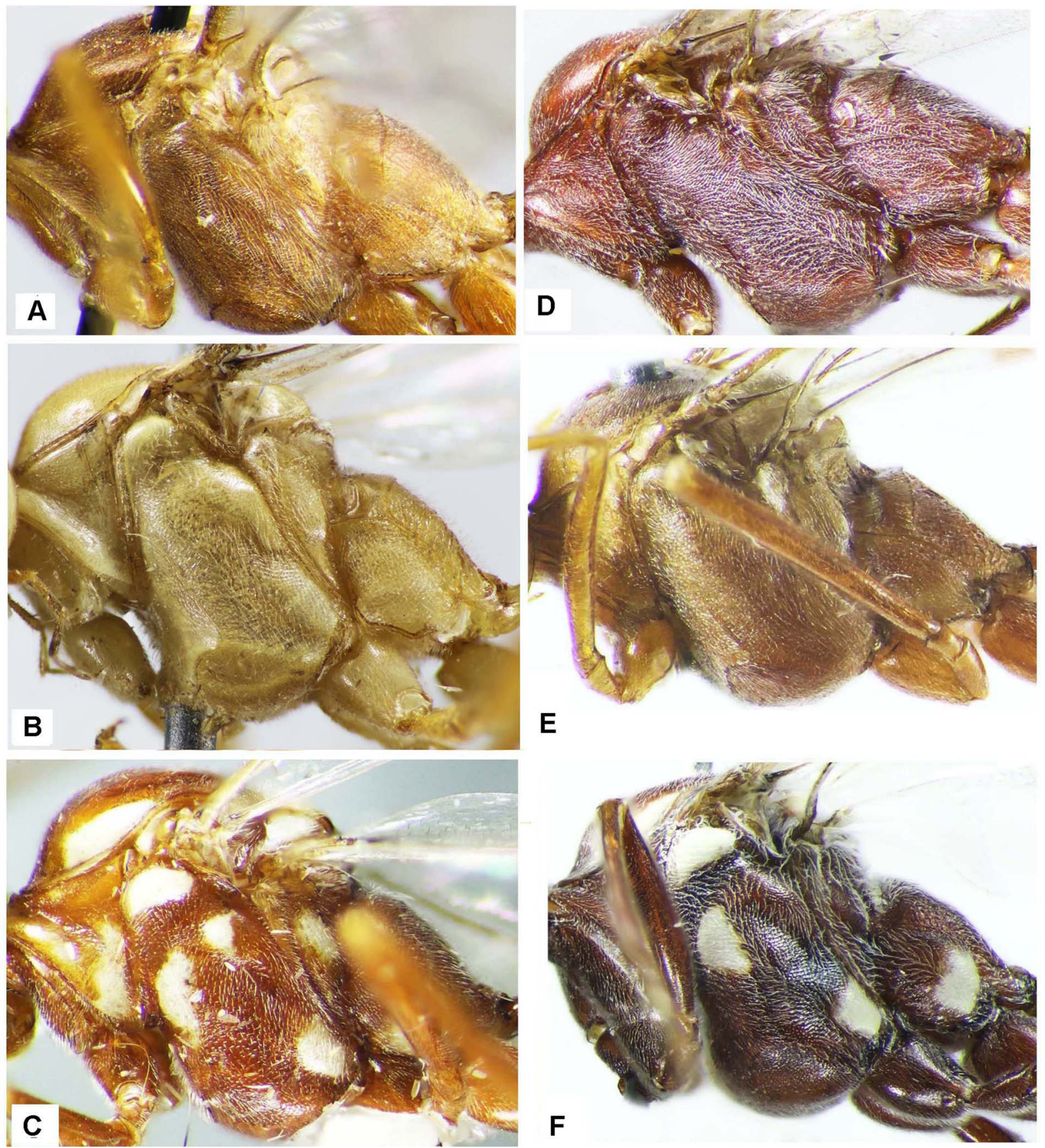

Fig. 16. Lateral aspect of mesosoma. A. Enicospilus pacificus (Holmgren, 1868). B. E. pallidus (Taschenberg, 1875). C. E. pseudoculator Gadallah \& Soliman sp. nov. D. E. rundiensis Bischoff, 1915. E. E. senescens (Tosquinet, 1896). F. E. shadaensis Gadallah \& Soliman sp. nov. 

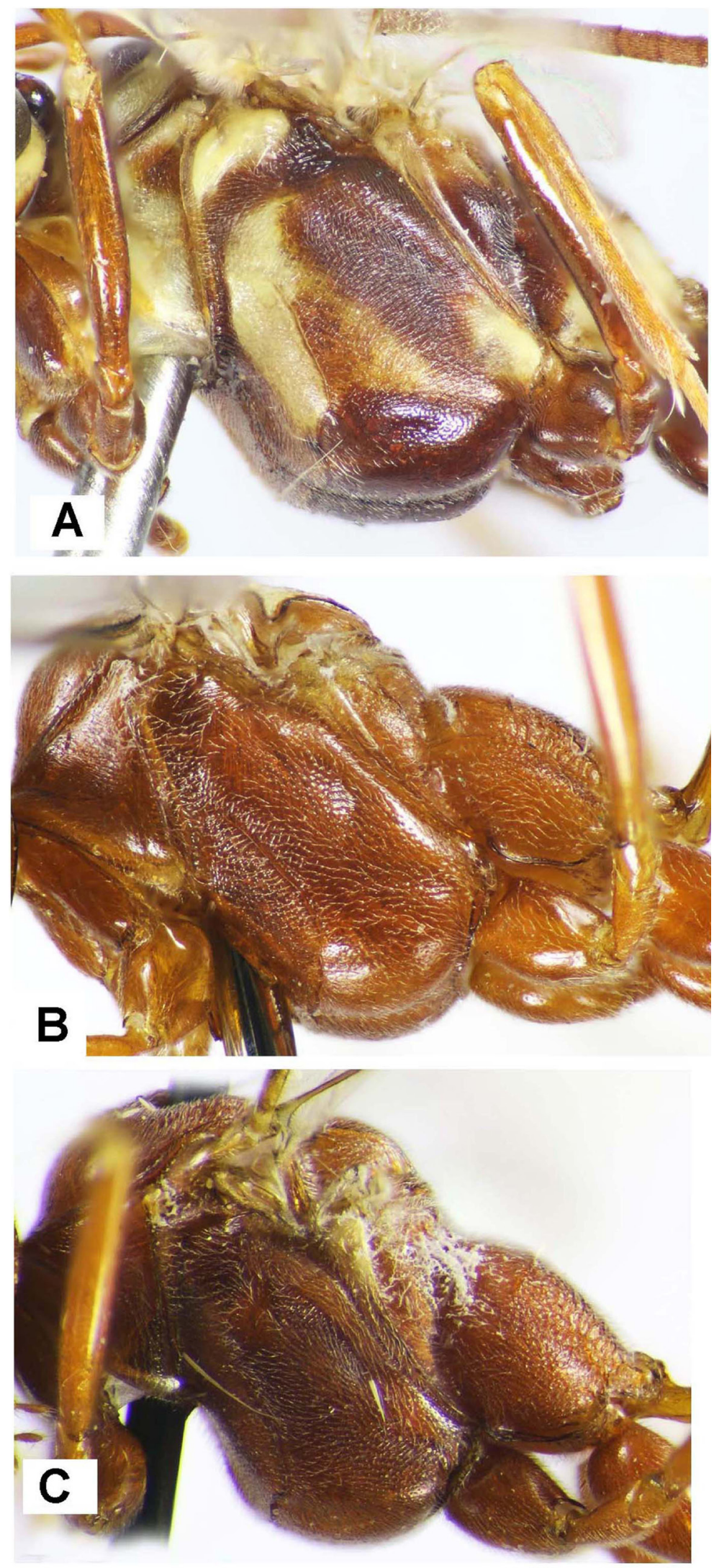

Fig. 17. Lateral aspect of mesosoma. A. Enicospilus splendidus Rousse, Soliman \& Gadallah sp. nov. B. Enicospilus sp. 2 cf. bicoloratus Cameron, 1915. C. Enicospilus sp. 1 
Enicospilus oweni Gauld \& Mitchell, 1978

Figs 4D, 7F, 11F, 15F, 19F, 25C, 30F

Enicospilus oweni Gauld \& Mitchell, 1978: 67, , ô.

Diagnosis (after Gauld \& Mitchell 1978)

B 21-23; F 13-15; ML 0.2; CT 1.4; OOL 0.02, POL 0.3; FI 0.45-0.6; Fl1-2 1.7-2.0; Fl20 1.9-2.1; AI 0.6-0.8; ICI 0.51-0.73; CI 0.5; SDI 1.19; NI 2.0.

Body reddish brown overall, vertex, face (including clypeus) and a narrow area along posterior border of head pale yellowish, mandibular teeth black; antennae reddish brown; legs yellowish orange; mandible dark and with subequal teeth; clypeus flat in profile, with ventral margin straight or truncate; face finely densely punctate, $1.1-1.25 \times$ as high as wide; antenna with 55-58 flagellomeres; basal transverse carina of propodeum distinct, anterior area of propodeum finely and superficially punctate, posterior area coarsely transversely striate; disco-submarginal cell without any alar sclerites; Rs $+2 \mathrm{r}$ straight; hind wing with 5-7 distal hamuli on R1; fore tibia sparsely spinose.

\section{Material examimed}

SAUDI ARABIA: 1 q, Raydah (Asir), light trap (House), 31 Jul. 2015, leg. Al Dhafer et al. (EFC); 1 , Raydah (Asir), light trap 3, 6 Jun. 2014, leg. Al Dhafer et al. (KSMA); 1 , , Raydah (Asir), light trap 7, 20 Oct. 2014, leg. Al Dhafer et al. (KSMA); 1 +, Raydah (Asir), light trap 6, 21 Oct. 2014, leg. Al Dhafer et al. (KSMA); 1 q, Raydah (Asir), light trap 6, 7 May 2015, leg. Al Dhafer et al. (KSMA); 1 , Raydah (Asir), light trap 9, 7 May 2015, leg. Al Dhafer et al. (KSMA).

\section{BOLD Identification Number}

ADB4116.

\section{Distribution}

Democratic Republic of Congo, Ivory Coast, Nigeria, Sierra Leone, Yemen (Gauld \& Mitchell 1978; Yu et al. 2012); Saudi Arabia (new record).

Enicospilus pacificus (Holmgren, 1868)

Figs 4E, 8A, 12A, 16A, 20A, 26A, 31A

Ophion pacificus Holmgren, 1868: ?, ․ .

Diagnosis (after Gauld \& Mitchell 1978)

B 17-20; F 11-17; ML 0.26-0.3; CT 1.2-1.7; OOL 0.1, POL 0.7; FI 0.55; F11-2 1.6-2.3; Fl 20 2.1-2.3; AI 0.45-0.7; ICI 0.45-0.57; CI 0.31-0.41; SDI 1.38; NI 2.0.

Body reddish brown, but antennae, legs, posterior margin of eye, frons and metasoma are lighter; mandible with upper tooth $1.2 \times$ as long as lower tooth; clypeus convex in profile, with ventral margin in-turned; face 1.17-1.3 $\times$ as high as wide; antenna with 57-62 flagellomeres; mesopleuron punctostriate, metapleuron densely coarsely punctate; basal transverse carina of propodeum distinct; anterior area of propodeum superficially punctate and densely pubescent, posterior area finely longitudinally rugose especially medially, densely clothed with fine, suberect pale pubescence; proximal sclerite rather narrow, comma-shaped with very long distal extension, central sclerite small, uniformly sclerotized; hind wing with 5-8 distal hamuli on R1; fore tibia not spinose. 


\section{Material examined}

SAUDI ARABIA: 2 q $q$, Raydah (Asir), light trap 5, 26 Aug. 2014, leg. Al Dhafer et al. (EFC); 1 , Raydah (Asir), light trap 2, 26 Aug. 2014, leg. Al Dhafer et al.; 1 ㅇ, 1 ô, Al Kharj (Loulou Farm), sweep net, 26 Nov. 2008, leg. Hassan (EFC), 1 đૈ, Education Farm (Riyadh), sweep net, 19 May 2009, leg. Al Sobaae \& Mosbet (KSMA); 1 गे, Thee Ain (Al Baha), sweep net, 10 Mar. 2012, leg. El Torkey A. (KSMA); 1 , Shada Al Ala (Al Baha), light trap 3, Nov. 2013, leg. Al Dhafer et al. (KSMA); 1 , Shada Al Ala (Al Baha), light trap 6, 15 Feb. 2014, leg. Al Dhafer et al. (KSMA); 2 우, Raydah (Asir), light trap 8, 21 Feb. 2014, leg. Al Dhafer et al. (KSMA); 3 ふึ, 2 우, Raydah (Asir), light trap 9, 21 Feb. 2014, leg. Al Dhafer et al. (KSMA); 1 d, Wadi Rida (Asir), sweep net, 24 Feb. 2014, leg. Sharaf

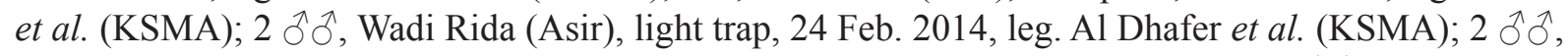
Shada Al Ala (Al Baha), light trap 1, 21 Apr. 2014, leg. Al Dhafer et al. (KSMA); $2 \hat{\jmath} \delta, 1$ q, Raydah (Asir), light trap 4, 27 Apr. 2014, leg. Al Dhafer et al. (KSMA); 1 J, Raydah (Asir), light trap 8, 27 Apr. 2014, leg. Al Dhafer et al. (KSMA); 1 क, Raydah (Asir), light trap 1, 6 Jun. 2014, leg. Al Dhafer et al. (KSMA); 2 우, Raydah (Asir), light trap 3, 6 Jun. 2014, leg. Al Dhafer et al. (KSMA); 1 으, Raydah (Asir), light trap 6, 6 Jun. 2014, leg. Al Dhafer et al. (KSMA); 1 ô, Raydah (Asir), light trap 7, 6 Jun. 2014, leg. Al Dhafer et al. (KSMA); 1 q, Raydah (Asir), light trap 1, 26 Aug. 2014, leg. Al Dhafer et al. (KSMA); 1 ㅇ, Raydah (Asir), light trap 9, 26 Aug. 2014, leg. Al Dhafer et al. (KSMA); 1 ○, Raydah (Asir), light trap 7, 20 Oct. 2014, leg. Al Dhafer et al. (KSMA); 1 \&, Raydah (Asir), light trap 4, 11 Dec. 2014, leg. Al Dhafer et al. (KSMA); 1 đ, Raydah (Asir), light trap 9, 5 Mar. 2015, leg. Al Dhafer et al.

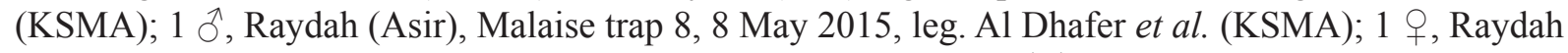
(Asir), light trap 7, 18 Nov. 2015, leg. Al Dhafer et al. (KSMA); $2 \hat{\jmath} \widehat{\partial}, 1$ q, Raydah (Asir), light trap 9, 17-18 Nov. 2015, leg. Al Dhafer et al. (KSMA).

\section{BOLD Identification Number}

ACX7776.

\section{Distribution}

Angola, Botswana, Burundi, Central African Republic, Congo, Ethiopia, Ivory Coast, Kenya, Madagascar, Nigeria, Rowanda, Sierra Leone, South Africa, Sudan, Tanzania, Uganda (Gauld \& Mitchell 1978); Saudi Arabia (new record).

Enicospilus pallidus (Taschenberg, 1875)

Figs 4F, 8B, 12B, 16B, 20B, 26B, 31B

Ophion pallidus Taschenberg, 1875: 436, ㅇ.

Henicospilus damarensis Cameron, 1906: 81, 9 .

Henicospilus sinicarinatus Enderlein, 1914: 218, ठิ.

Henicospilus techowi Enderlein, 1918: 219, गे.

Henicospilus dinteri Enderlein, 1918: 220, ㅇ.

Diagnosis (after Gauld \& Mitchell 1978)

B 22; F 12-15; ML 0.27; CT 1.66; OOL 0.04, POL 0.5; FI 0.5; F11-2 1.78; Fl20 1.5; AI 0.8; ICI 0.66; CI 0.33 ; SDI 1.27 ; NI 2.5 .

Body including coxae pale yellow overall, metasoma slightly darker ventrally; mesoscutum with median brown stripe extending over anterior half; remaining parts of legs light brown; pterostigma yellowish; proximal and distal wing sclerites yellowish brown; mandible with upper tooth $1.3 \times$ as long as lower tooth; clypeus flat in profile, ventral margin truncate; face $1.2 \times$ as high as wide; antenna with 60-65 flagellomeres; mesopleuron finely puncto-striate, metapleuron finely punctate; basal transverse carina of propodeum distinct, anterior area smooth, posterior area irregularly arcuately wrinkled; disco- 
submarginal cell of fore wing with triangular proximal sclerite, central sclerite crescent-shape, weakly sclerotized proximally and large, with maximal length larger than distance to Rs +2 r; hind wing with 7 distal hamuli on R1; fore tibia sparsely spinose.

\section{Material examined}

SAUDI ARABIA: 1 + , Shada Al Ala (Al Baha), light trap 6, 15 Feb. 2014, leg. Al Dhafer et al. (KSMA).

\section{BOLD Identification Number}

\section{AAI5153.}

\section{Distribution}

Namibia, Sierra Leone (Gauld \& Mitchell 1978), Chad, Ethiopia, Kenya, South Africa, Sudan (Gauld \& Mitchell 1978; Yu et al. 2012), Democratic Republic of Congo, Pakistan (Yu et al. 2012), Tanzania (Rousse \& van Noort 2014); Saudi Arabia (new record).

Enicospilus perlatus Shestakov, 1926

Fig. 34B

Enicospilus perlatus Shestakov, 1926: 30, ․

\section{Diagnosis}

B 26; F 16; ML 0.8; CT 2.0; OOL 0.2, POL 1.0; FI 0.37; AI 0.4; ICI 0.7 ; CI 0.05; SDI 1.16; NI 1.0-1.2.

Body dark reddish brown overall, with the head yellow (except a red longitudinal band on lower face including clypeus), mesoscutum reddish brown with yellow U-shape, with broad base as well as lateral margin, lateral red lobes of mesoscutum with a longitudinal black line extending along scutellum which is yellow with small red marking basally, propodeum and metasoma (except ventrally black) entirely dark reddish brown, all legs bright red, metasomal T1 and T2 brighter in color, mandible reddish except tip black, meso- and metapleura with traces of yellowish markings (two on mesopleuron and one distally on metapleuron); mandible with upper tooth $2.0 \times$ as long as lower tooth; clypeus flat in profile, distinctly wider than long, with ventral margin truncate; antenna with 57 flagellomeres; mesopleuron and metapleuron puncto-striate; propodeum densely covered with fine setae, basal transverse carina of propodeum weak, hardly visible laterally, anterior area nearly smooth to finely superficially punctate, posterior area coarsely rugose longitudinally, especially medially; proximal sclerite bright red, domeshaped, central sclerite small, slightly elongate and totally transluscent, distal sclerite crescent shaped, enclosing central one; fore tibia sparsely spinose.

\section{Material examined}

SAUDI ARABIA: 1 q, Hedjaz (Mastoura), 1 Feb. 1937, leg.? (PPDD) (in bad condition).

\section{Previous records from Saudi Arabia}

Riyadh (Horstmann 1981).

\section{Distribution}

Afghanistan, Canary Islands, Morocco (Yu et al. 2012), Egypt (Shaumar 1966), Iran (Hedwig 1957 as E. tricolor; Kolarov \& Ghahari 2005; Barahoei et al. 2012), Israel (Aubert et al. 1984; Yu et al. 2012), Spain (Bordera et al. 1987), Turkmenistan (Shestakov 1926; Kolarov \& Ghahari 2005), Iran (Hedwig 1957 as E. tricolor), Saudi Arabia (Horstmann 1981 as E. tricolor). 
Enicospilus psammus Gauld \& Mitchell, 1978

Fig. 34A

Enicospilus psammus Gauld \& Mitchell, 1978: 147, 148.

Diagnosis (after Gauld \& Mitchell 1978)

F 6.0-9.0; ML 0.1; OOL 0.04, POL 0.5; FI 0.5-0.55; Fl 1.8-2.0; Fl20 1.3-1.4; AI 0.5-0.6; ICI 0.3-0.4; CI 0.3-0.4; SDI 1.2-1.3.

Body generally reddish brown, in some cases with propodeum and metasoma infuscate, lower face, interocellar area and genae yellowish brown; mandible with upper tooth 2.0-3.0 $\times$ as long as lower tooth; clypeus convex in profile, with impressed margin, with ventral margin truncate; lower face subquadrate, $0.8-0.9 \times$ as broad as long; antenna with 44-48 flagellomeres; mesopleuron with upper area puncto-striate, while lower area coarsely punctate; metapleuron puncto-striate; basal transverse carina of propodeum complete, anterior area of propodeum striate, posterior area irregularly wrinkled; fore wing with three sclerites, proximal sclerite dome- to triangular in shape, central sclerite transversely oval, while distal sclerite small, comma-shaped; hind wing with 5-6 hamuli on R1; fore tibia with scattered spines.

\section{Material examined}

None.

\section{Previous records from Saudi Arabia}

Abian, Al Lith, Asir, Qunfida (Gauld \& Mitchell 1978), Bahara (Horstmann 1981).

\section{Distribution}

Saudi Arabia (Gauld \& Mitchell 1978; Horstmann 1981), Sudan (Gauld \& Mitchell 1978).

Enicospilus pseudoculator Gadallah \& Soliman sp. nov. urn:1sid:zoobank.org:act:0155506A-1D33-4CFA-8456-1B342A1032BE

Figs 4G, 8C, 12C, 16C, 20C, 26C, 31C

\section{Diagnosis}

Dark reddish brown overall, including antennae and legs, with ivory on head and mesosoma as follows: posterior margin of head, all face except medially just from behind fore ocellus (between antennal bases) to base of clypeus (reddish brown), sides of mesoscutum (until tegula, not extending to posterior margin), tegula, two upper and lower mesopleural markings, a large posterior marking on metapleuron (just above hind coxa); mandible with upper tooth $2.0 \times$ as long as lower tooth; clypeus flat in profile, ventral margin truncate; face subquadrate, $1.1 \times$ as high as wide; antenna with 42-44 flagellomeres; mesopleuron and metapleuron finely and densely punctate; basal transverse carina of propodeum distinct, anterior area shallowly punctate to smooth, posterior area shallowly transversely striate; proximal sclerite bright red and dome-shaped, central sclerite totally absent; hind wing with 6 distal hamuli on R1.

\section{Etymology}

Named with reference to the similarity of this species to Enicospilus oculator.

\section{Type material}

\section{Holotype}

SAUDI ARABIA: ㅇ, Raydah (Asir), light trap (House), 18 $13.347^{\prime} \mathrm{N}, 42^{\circ} 24.133^{\prime} \mathrm{E}$, alt. $2717 \mathrm{~m}$, 26 Aug. 2014, leg. Al Dhafer et al. (KSMA). 


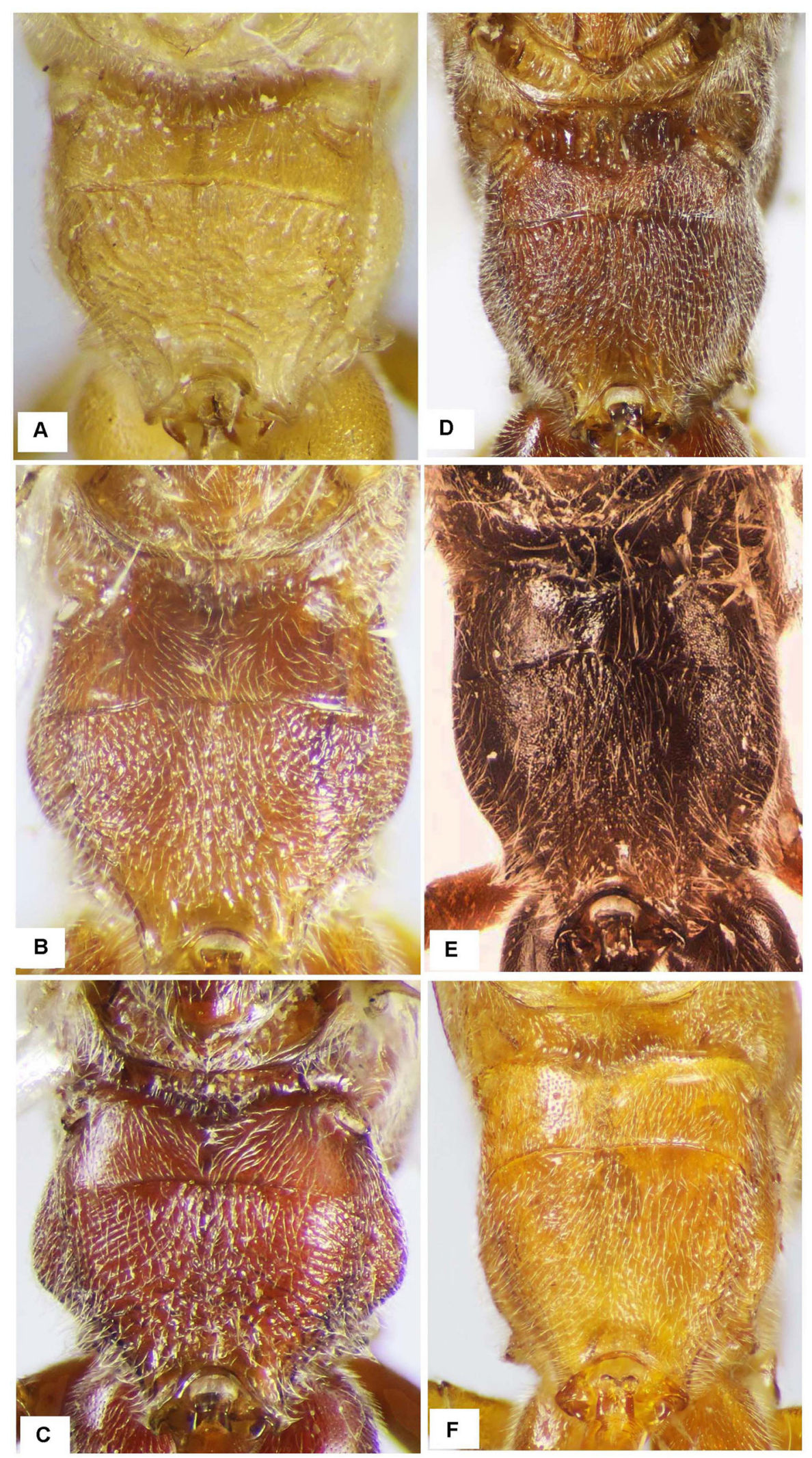

Fig. 18. Propodeum. A. Enicospilus arabicus Gadallah \& Soliman sp. nov. B. E. bicoloratus Cameron, 1912. C. E. brevicornis (Masi, 1939). D. E. capensis (Thunberg, 1822). E. E. divisus (Seyrig, 1935). F. E. dubius (Tosquinet, 1896). 

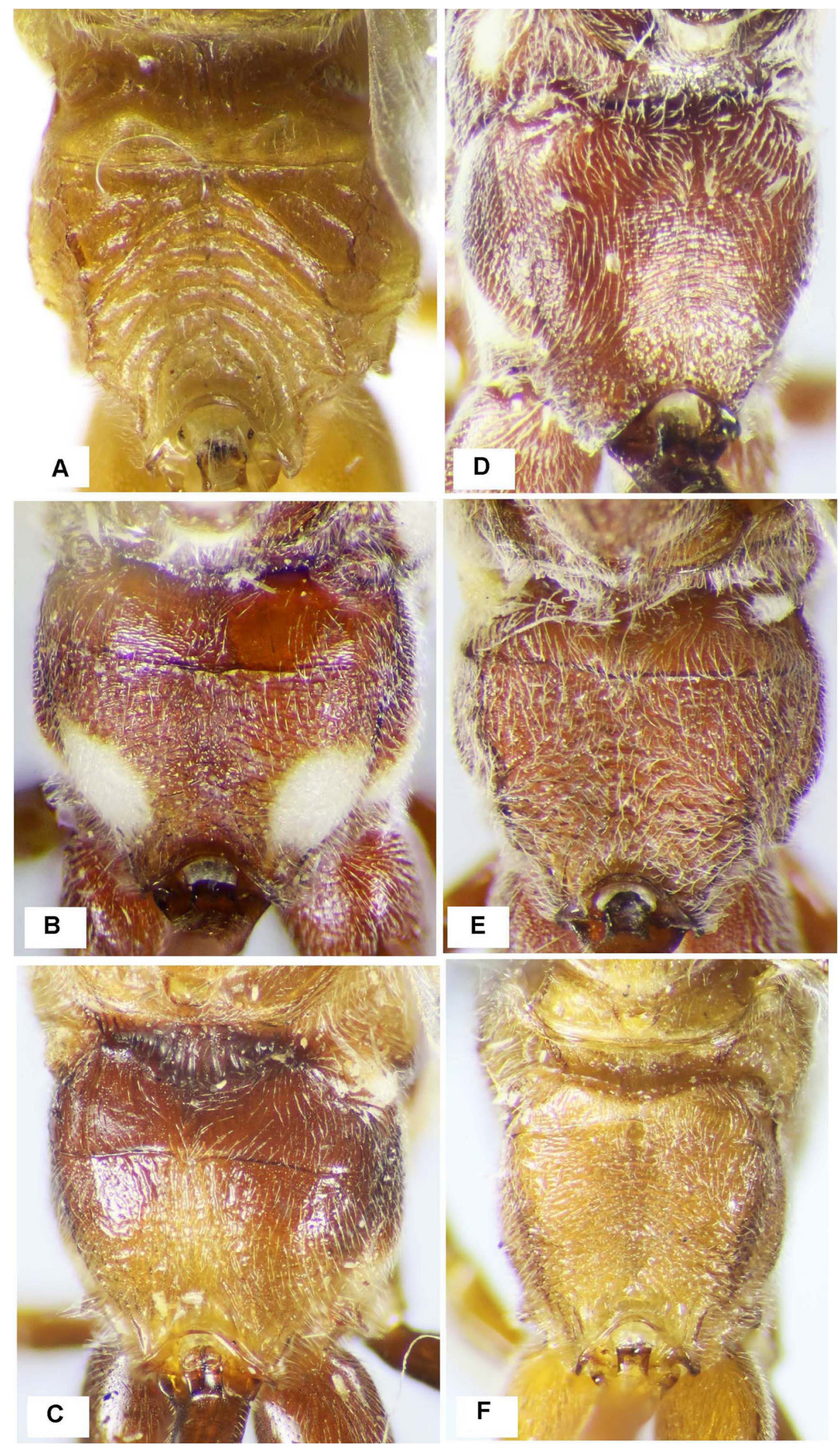

Fig. 19. Propodeum. A. Enicospilus grandiflavus Townes \& Townes, 1973. B. E. mirabilis Soliman \& Gadallah sp. nov. C. E. nervellator Aubert, 1966. D. E. oculator Seyrig, 1935. E. E. odax Gauld \& Mitchell, 1978. F. E. oweni Gauld \& Mitchell, 1978. 

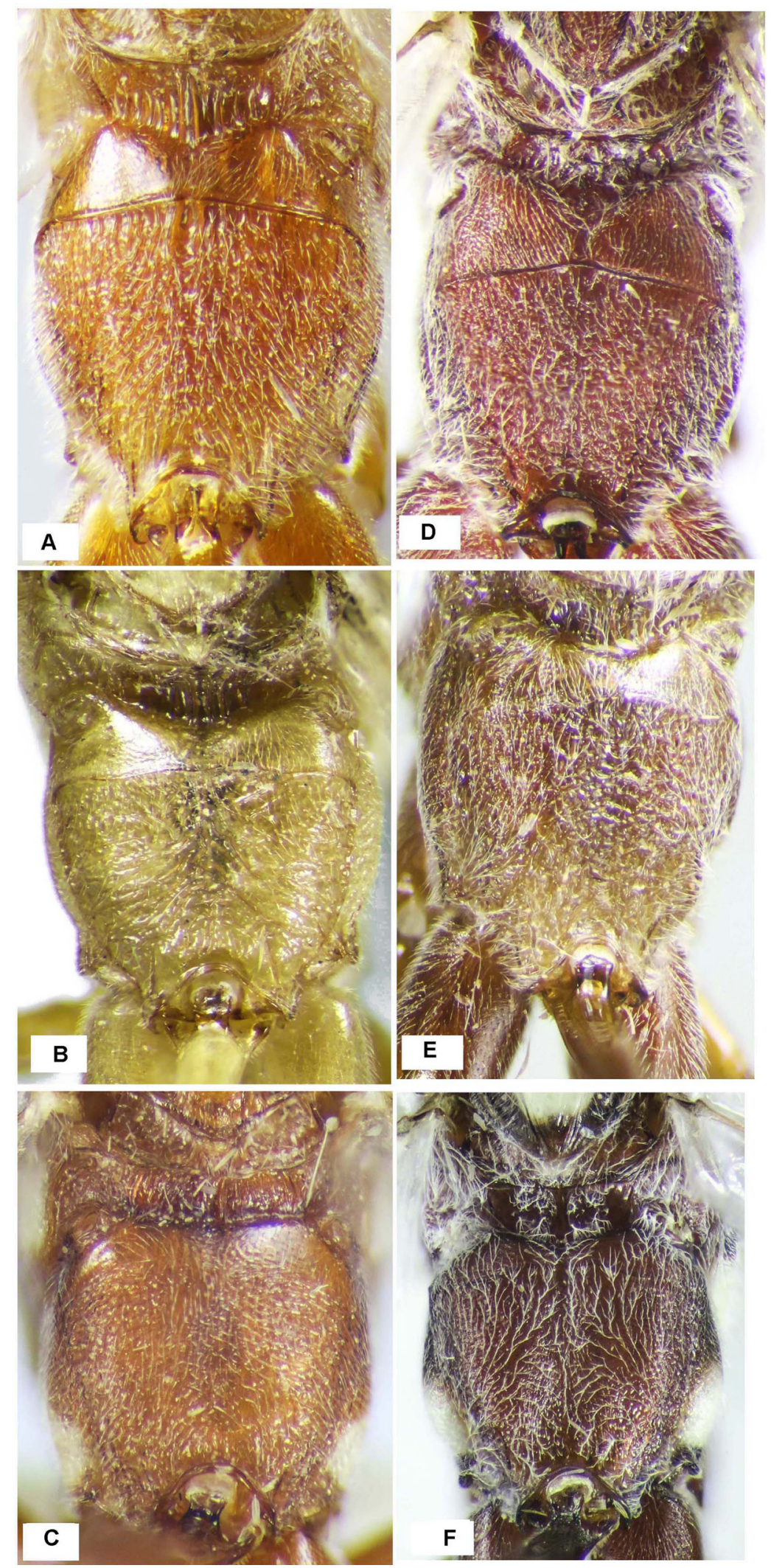

Fig. 20. Propodeum. A. Enicospilus pacificus (Holmgren, 1868). B. E. pallidus (Taschenberg, 1875). C. E. pseudoculator Gadallah \& Soliman sp. nov. D. E. rundiensis Bischoff, 1915. E. E. senescens (Tosquinet, 1896). F. E. shadaensis Gadallah \& Soliman sp. nov. 


\section{Paratypes}

SAUDI ARABIA: 1 o, Rhodet Khorim (Riyadh), light trap (B), 14 Apr. 2012, leg.? (EFC); 1 क,

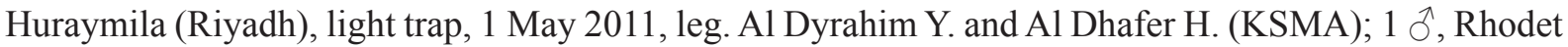
Khorim (Riyadh), light trap (B), 14 Apr. 2012, leg. Al Dhafer et al. (KSMA); 1 , , Raydah (Asir), light trap 6, 27 Apr. 2014, leg. Al Dhafer et al. (KSMA); 2 q , Raydah (Asir), light trap 4, 6 Jun. 2014, leg. Al Dhafer et al. (KSMA); 1 q, Raydah (Asir), light trap 6, 26 Aug. 2014, leg. Al Dhafer et al. (KSMA); 1 , Shada Al Ala (Al Baha), light trap 3, 2 Mar. 2015, leg. Al Dhafer et al. (KSMA); 1 + , Wadi Hanifa (Riyadh), light trap 3, 2 Apr. 2015, leg. Al Dhafer et al. (KSMA); 1 o, 1 \&, Rawdet Al Harmalyiah (Riyadh), light trap 3, 17 Jun. 2015, leg. Al Dhafer et al. (KSMA); 1 क, Raydah (Asir), light trap 4, 5 Nov. 2015, leg. Al Dhafer et al. (KSMA).

\section{Description}

Female ( 9 specimens)

B 15-17; F 12-14; ML 0.5; CT 1.4; OOL 0.1; POL 0.66; FI 0.56; Fl1-2 1.5; Fl 20 1.66; AI 0.9; ICI 0.68; CI 0.16; SDI 1.1; NI 2.25.

CoLour. Dark reddish brown overall, including antennae and legs, with ivory on head and mesosoma as follows: posterior margin of head, all face except in middle just from behind fore ocellus (between antennal bases) to base of clypeus (dark reddish brown), sides of mesoscutum (until tegula), under fore and hind wings, two upper and lower mesopleural markings, a large posterior marking on metapleuron (just above hind coxa).

HeAD. Mandible moderately twisted, upper tooth distinctly longer than lower tooth, $(2.0 \times$ as long as lower tooth), broad at base and narrowed apically, without groove on outer surface, with fine dense setae; labrum $0.4-0.5 \times$ as high as wide; clypeus flat in profile, ventral margin truncate; face and clypeus finely but somewhat sparsely punctate; lower face subquadrate, $1.1 \times$ as long as broad; gena strongly constricted behind eyes; occipital carina complete; ocelli distinctly enlarged; antenna with 43-44 flagellomeres.

Mesosoma. Pronotum normal, not visible in dorsal view; mesoscutum densely superficially punctate; epicnemial carina distinct to level of ventral corner of pronotum; postpectal carina weak, just visible
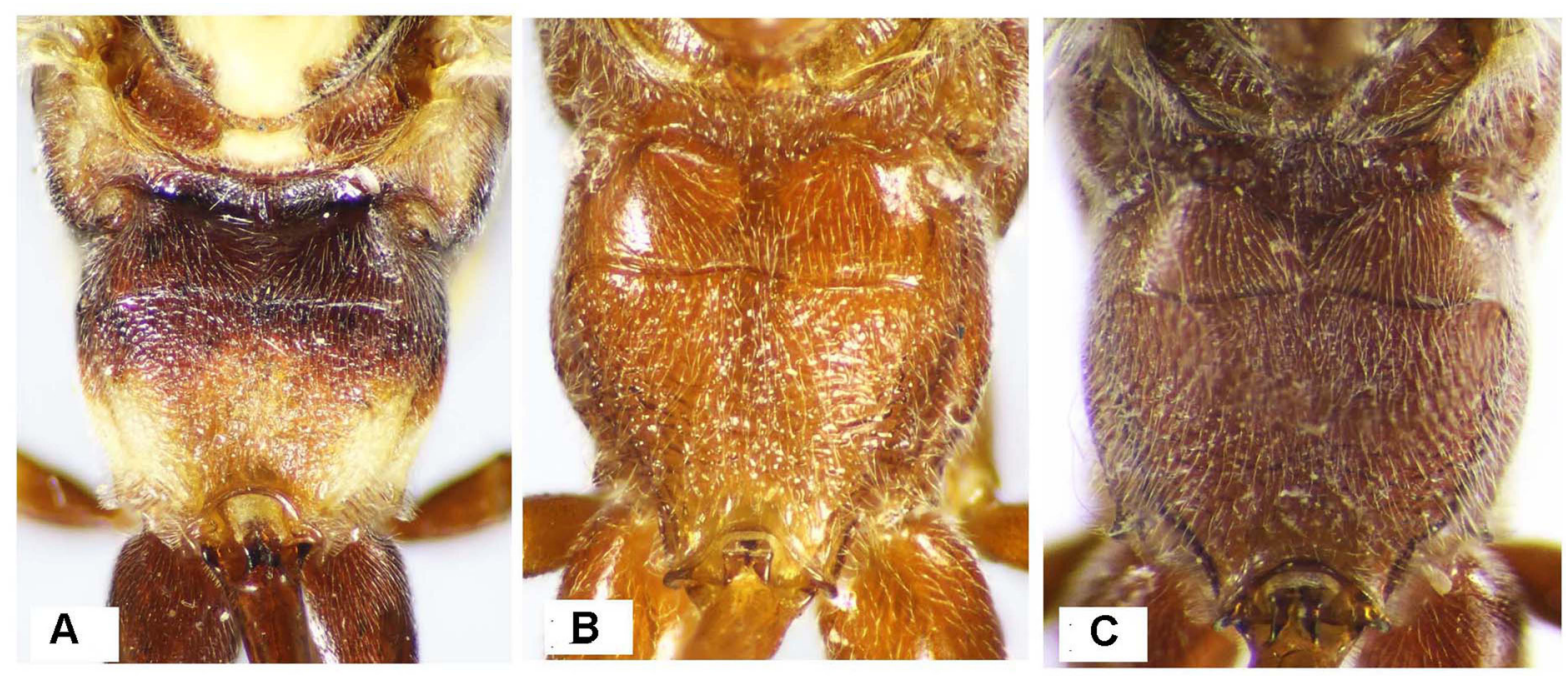

Fig. 21. Propodeum. A. Enicospilus splendidus Rousse, Soliman \& Gadallah sp. nov. B. Enicospilus sp. 2 cf. bicoloratus Cameron, 1915. C. Enicospilus sp. 1 
laterally while indistinct medially; notauli indistinct; scuto-scutellar groove strong, smooth; scutellum shallowly punctate, slightly longer than its basal width, carinate to near apex; meso- and metapleura densely, finely punctate; basal transverse carina of propodeum weak but distinct especially medially, anterior area shallowly punctate to smooth, posterior area shallowly transversely striate.

WINGS. Proximal sclerite bright red and dome-shaped, central sclerite totally absent; Rs $+2 \mathrm{r}$ straight, not thickened; hind wing with 6 distal hamuli on R1.

Legs. Fore tibia sparsely setose; hind coxa elongate, $2.0 \times$ as long as high; hind trochantellus middorsally $1.2 \times$ as long as broad; hind tarsal claw symmetrical, with 11-12 equal-sized teeth.

Metasoma. Laterally flattened, widened posteriorly; T2 in profile about $3.5 \times$ as long as high; thyridium deep, bar-shaped, extending along basal third of T2.

Male (4 specimens)

B 14; F 9.

Resembles female but differs in the following: head and mesosoma brighter red while metasoma dark reddish brown; ivory markings on mesosoma larger, tegula marked with ivory; mesoscutum laterally with ivory line extending along whole length of it, ending with two large rounded markings, two thin middle lines (absent in female); hind wing with 5 distal hamuli on R1.

\section{BOLD Identification Number}

ADB4338.

\section{Remarks}

This species closely resembles E. oculator except for the following: central sclerite totally absent (in oculator weakly sclerotized proximally); CI 0.16 (in oculator CI 0.25 ).

\section{Distribution}

Saudi Arabia.

Enicospilus rundiensis Bischoff, 1915

Figs 4H, 8D, 12D, 16D, 20D, 27A, 31D

Enicospilus (Dispilus) rundiensis Bischoff, 1915: 476, ô.

Enicospilus ruandensis Roman, 1924: 8, ․

Diagnosis (after Gauld \& Mitchell 1978)

B 18-21; F 12-16; ML 0.2; CT 1.4-1.8; OOL 0.1, POL 0.29; FI 0.55-0.6; Fl1-2 1.4-1.6; Fl20 1.8-2.0; AI 0.55-0.75; ICI 0.45-0.7; CI 0.45-0.65; SDI 1.3-1.4; NI 2.37.

Body reddish brown overall, orbits pale yellow, gena yellowish white, ventral side of T3-6 darker in colour, antennae and legs are brighter in colour, mandibular teeth black; mandible with upper tooth 1.5$2.0 \times$ as long as lower tooth; clypeus slightly convex in profile, ventral margin in-turned; face $1.25 \times$ as high as wide; antenna with 57-61 flagellomeres; mesopleuron finely puncto-striate, metapleuron finely punctate; basal transverse carina of propodeum distinct, anterior area of propodeum finely superficially shagreened, posterior area coarsely closely rugose; proximal sclerite triangular, dark brown, central sclerite moderately to very long and curved toward base of wing; hind wing with 6-8 distal hamuli on $\mathrm{R} 1$; fore tibia sparsely setose. 


\section{Material examined}

SAUDI ARABIA: 1 + , Shada Al Ala (Al Baha), light trap 1, 27 Jan. 2015, leg. Al Dhafer et al. (KSMA); 1 , Raydah (Asir), light trap 6, 30 Jan. 2015, leg. Al Dhafer et al. (KSMA); 6 우, 1 ㄱ, Raydah (Asir), light trap 8, 30 Jan. 2015, leg. Al Dhafer et al. (KSMA); 11 우, Raydah (Asir), light trap 9, 30 Jan. 2015, leg. Al Dhafer et al. (KSMA).

\section{Description}

Males of E. rundiensis resemble the female (see diagnosis).

\section{BOLD Identification Number}

ADB5118.

\section{Distribution}

Burundi, Democratic Republic of Congo, Ethiopia, Kenya, Rwanda (Gauld \& Mitchell 1978; Yu et al. 2012), Namibia, Zimbabwe (Rousse \& van Noort 2014); Saudi Arabia (new record).

Enicospilus senescens (Tosquinet, 1896)

Figs 5A, 8E, 12E, 16E, 20E, 27B, 31E

Ophion (Allocamptus) senescens Tosquinet, 1896: 375, ô.

O. (Allocamptus) infuscatus Tosquinet, 1896: 373, ô.

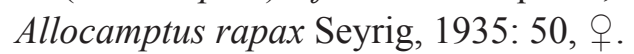

Diagnosis (after Gauld \& Mitchell 1978)

B 18-20; F 11-17; ML 0.1-0.2; CT 1.4-1.8; OOL 0.1, POL 0.45; FI 0.6-0.7; F11-2 1.5-1.6; F120 2.5-2.7; AI 1.1-1.65; ICI 0.7-1.0; CI 0.8-1.0; SDI 1.6-1.8; NI 2.5.

Body entirely reddish to orange, orbits pale yellowish; mandible with upper tooth about $1.25 \times$ as long as lower tooth; clypeus slightly convex in profile, ventral margin slightly in-turned; face $1.2 \times$ as long as wide; antenna with 51-62 flagellomeres; meso- and metapleura puncto-striate; basal transverse carina of propodeum distinct, anterior area of propodeum nearly smooth to superficially shagreened, posterior area coarsely reticulate; disco-submarginal cell of fore wing without alar sclerites, Rs $+2 \mathrm{r}$ strongly sinuate proximally; hind wing with 5-8 distal hamuli on R1; fore tibia sparsely setose.

\section{Material examined}

SAUDI ARABIA: 2 q, , Raydah (Asir), light trap 6, 26 Aug. 2014, leg. Al Dhafer et al. (EFC); $1 \hat{\jmath}$, Raydah (Asir), light trap 9, 21 Feb. 2014, leg. Al Dhafer et al. (KSMA); 1 \%, Raydah (Asir), light trap 3, 20 Oct. 2014, leg. Al Dhafer et al. (KSMA); 1 9, Raydah (Asir), light trap 9, 30 Jan. 2015, leg. Al Dhafer et al. (KSMA); 1 , Raydah (Asir), light trap 4, 31 Jul. 2015, leg. Al Dhafer et al. (KSMA); 1 , Shada Al Ala (Al Baha), light trap 1, 2 Sep. 2015, leg. Al Dhafer et al. (KSMA); 1 ô, Raydah (Asir), light trap 7, 5 Sep. 2015, leg. Al Dhafer et al. (KSMA).

\section{Distribution}

Togo, Ghana, Ethiopia, Angola, Central African Republic, Guinea, Ivory Coast, Kenya, Madagascar, Malawi, Nigeria, Rwanda, Sierra Leone, Uganda (Gauld \& Mitchell 1978), Democratic Republic of Congo, Benin, South Africa, Zimbabwe (Yu et al. 2012), Gabon (Gauld \& Mitchell 1978; van Noort 2004); Saudi Arabia (new records). 
Enicospilus shadaensis Gadallah \& Soliman sp. nov. urn:lsid:zoobank.org:act:F2609434-0E0C-4ACB-BAE7-2A1FCB4F89CB

Figs 5B, 8F, 12F, 16F, 20F, 27C, 31F

\section{Diagnosis}

B 19-21, F 12-13; ML 0.2; CT 1.2; POL1.0; FI 0.65; Fl1-2 1.6; AI 0.87; CI 0.3; ICI 0.5; SDI 1.2; NI 3.8.

Moderately large sized and reddish brown overall including antennae and legs, with ivory yellow on face (except the middle broadly extending from behind the fore ocellus), posterior margin of eyes, gena, two pale rounded markings on lateral sides of clypeus, lateral sides of mesoscutum that are broad anteriorly, becoming very thin laterally, scutellum, subalar prominence, two markings on upper and lower ends of mesopleuron, upper one distinctly larger, a large one on lower part of metapleuron just above hind coxa. Metasoma with T1 entirely dark brown to black, T2 reddish brown with orange or lighter posterior margin, remaining tergites mostly light red to orange blackish medially, last tergite reddish with dark posterior end; mandible reddish with black teeth, upper tooth $2.0 \times$ as long as lower tooth; clypeus flat in profile, ventral margin truncate, antenna with 50 flagellomeres; meso- and metapleura puncto-striate; basal transverse carina of propodeum distinct, anterior area of propodeum nearly smooth, posterior area superficially reticulate rugose; proximal sclerite triangular, dark brown to black; fore tibia not spinose.

\section{Etymology}

The species epithet "shadaensis" refers to Shada Al Ala, one of the localities where specimens were collected.

\section{Type material}

\section{Holotype}

SAUDI ARABIA: ㅇ, Raydah (Asir), light trap $8,18^{\circ} 11.618^{\prime} \mathrm{N}, 42^{\circ} 23.420^{\prime} \mathrm{E}$, alt. $1772 \mathrm{~m}, 26$ Aug. 2014, leg. Al Dhafer et al. (KSMA).

\section{Paratypes}

SAUDI ARABIA: 1 , Shada Al Ala (Al Bahha), light trap 4, 8 Dec. 2014, leg. Al Dhafer et al. (EFC); 1 ô, Muzahmiah (Al Khararah), 17 May 2011, leg. Al Drayhim et al. (EFC); 1 o, Shada Al Ala (Al Baha), light trap 4, 14 Nov. 2015, leg. Al Dhafer et al. (EFC); 1 ô, Wadi Turubah (Al Baha), light trap, 1 Jun. 2011, leg. Al Dhafer H. and Al-Gharabawi A. (KSMA); 1 ô, Shada Al Ala (Al Baha), light trap (House), 15 Feb. 2014, leg. Abdel-Dayem M. and Rasool I. (KSMA); 1 + , Raydah (Asir), light trap 9, 21 Feb. 2014, leg. Al Dhafer et al. (KSMA); 1 đ, Raydah (Asir), light trap 7, 26 Aug. 2014, leg. Al Dhafer et al. (KSMA); 1 + , Raydah (Asir), light trap 9, 20 Oct. 2014, leg. Al Dhafer et al. (KSMA); 1 o, Shada Al Ala (Al Baha), light trap 5, 27 Jan. 2015, leg. Al Dhafer et al. (KSMA); 1 ô, 1 ㅇ, Shada Al Ala (Al Baha), light trap 4, 14 Nov. 2015, leg. Al Dhafer et al. (KSMA); 2 우, Shada Al Ala (Al Baha), light trap 2, 18 Nov. 2015, leg. Al Dhafer et al. (KSMA).

\section{Description}

Female (7 specimens)

B 19-21; F 12-13; ML 0.2; CT 1.2; POL1.0; FI 0.65; Fl1-2 1.6; AI 0.87; CI 0.3; ICI 0.5; SDI 1.2; NI 3.8.

CoLour. Reddish brown overall including antennae and legs, with ivory yellow on vertex, orbits, lateral sides of mesoscutum (which are broad anteriorly, becoming very thin laterally, but not reaching end) scutellum, subalar prominence, two markings on upper and lower ends of mesopleuron, upper one distinctly larger, a large marking on lower part of metapleuron (just above hind coxa); metasoma with $\mathrm{T} 1$ entirely dark brown to black, T2 with lighter posterior margin, remaining metasomal tergites mostly light red to orange, blackish medially, last tergite reddish, with dark posterior margin; mandible reddish 
with black teeth; fore wing with dark to black pterostigma with yellow proximal corner, parastigma yellow, distal end of pterostigma pale brown, sclerites and wing venation dark brown.

HEAD. Mandible hardly twisted, parallel-sided, with black thin outer margin, upper tooth about $2.0 \times$ as long as lower tooth, outer mandibular surface with dense fine pale setae, without groove on outer surface; clypeus superficially punctate, flat in profile, ventral margin truncate; labrum semicircular, about $0.3 \times$ as high as wide; face $1.3 \times$ as high as wide; gena constricted behind eyes; occipital carina complete and thin; ocelli relatively large, lateral one nearly touching eyes; antenna with 50 flagellomeres.

Mesosoma. Pronotum normal, with deep transverse furrow; epicnemial carina more or less distinct, weak; postpectal carina only distinct laterally, interrupted or indistict medially; mesoscutum densely punctate-reticulate, notauli indistinct; scutellum smooth, impunctate, slightly longer than basal width, carinate to apex; mesopleuron and metapleuron densely punctate; basal transverse carina of propodeum weak, anterior area of propodeum nearly smooth, posterior area shallowly rugose.

Wings. Disco-submarginal cell of fore wing with well developed fenestra, proximal sclerite triangular, dark brown to black; central sclerite totally absent; Rs $+2 \mathrm{r}$ slightly thickened ventrally at basal third, more or less straight; cu-a subopposite Rs \& M by about $0.25 \times$ length of cu-a; hind wing with 7 distal hamuli on R1.

Legs. Fore tibia not spinose; claws with black teeth, denser and shorter basally.

Metasoma. Slender; T2 in profile about $4 \times$ as long as high; thyridium very shallow, linear basally and widened apically, slightly posterior to one third length of tergite.

Male ( 7 specimens)

B 23; F 16.

Resembles female except for the following: body more slender; antenna with 52 flagellomeres; ivory markings on meso- and metapleura are distinctly smaller in size. Otherwise very similar to female.

\section{BOLD Identification Number}

\section{ABX3540.}

\section{Remarks}

This species agrees completely with the key characters of Enicospilus sp. 4 in Gauld \& Mitchell (1978). But compared to the full description, it differs in the following characters: antenna with 50 flagellomeres (in sp. 4 62-64 flagellomeres); alar sclerite resembles that of sp. 4 (Gauld \& Mitchell 1978: fig. 379); the mesoscutal ivory margins do not extend the whole length of the mesoscutum (in G. \& M. it extends along the whole margins of the mesoscutum); no central ivory marking could be seen on the mesopleuron of our specimen (in G. \& M. a central marking is present); metapleuron with a posterior ivory marking (in G. \& M. it is present centrally); T1 entirely black (not so in G. \& M.).

Enicospilus shadaensis sp. nov. resembles E. pseudoculator sp. nov. but differs in the following characters: body dark reddish brown, metasoma patterned with bright red to orange (in pseudoculator sp. nov. lighter orange to brown, metasoma not patterned with red); proximal sclerite dark brown to black, triangular (in pseudoculator sp. nov. proximal sclerite bright red and dome-shaped); face narrow, $1.5 \times$ as high as wide (in pseudoculator sp. nov. face subquadrate, $1.1 \times$ as high as wide); CI $>0.2$ (in pseudoculator sp. nov. $\mathrm{CI}<0.2)$; moderately large species $(\mathrm{B}>27 ; \mathrm{F}>12)$ (pseudoculator sp. nov. smaller species $(\mathrm{B}<19, \mathrm{~F}<12)$ ).

\section{Distribution}

Saudi Arabia. 

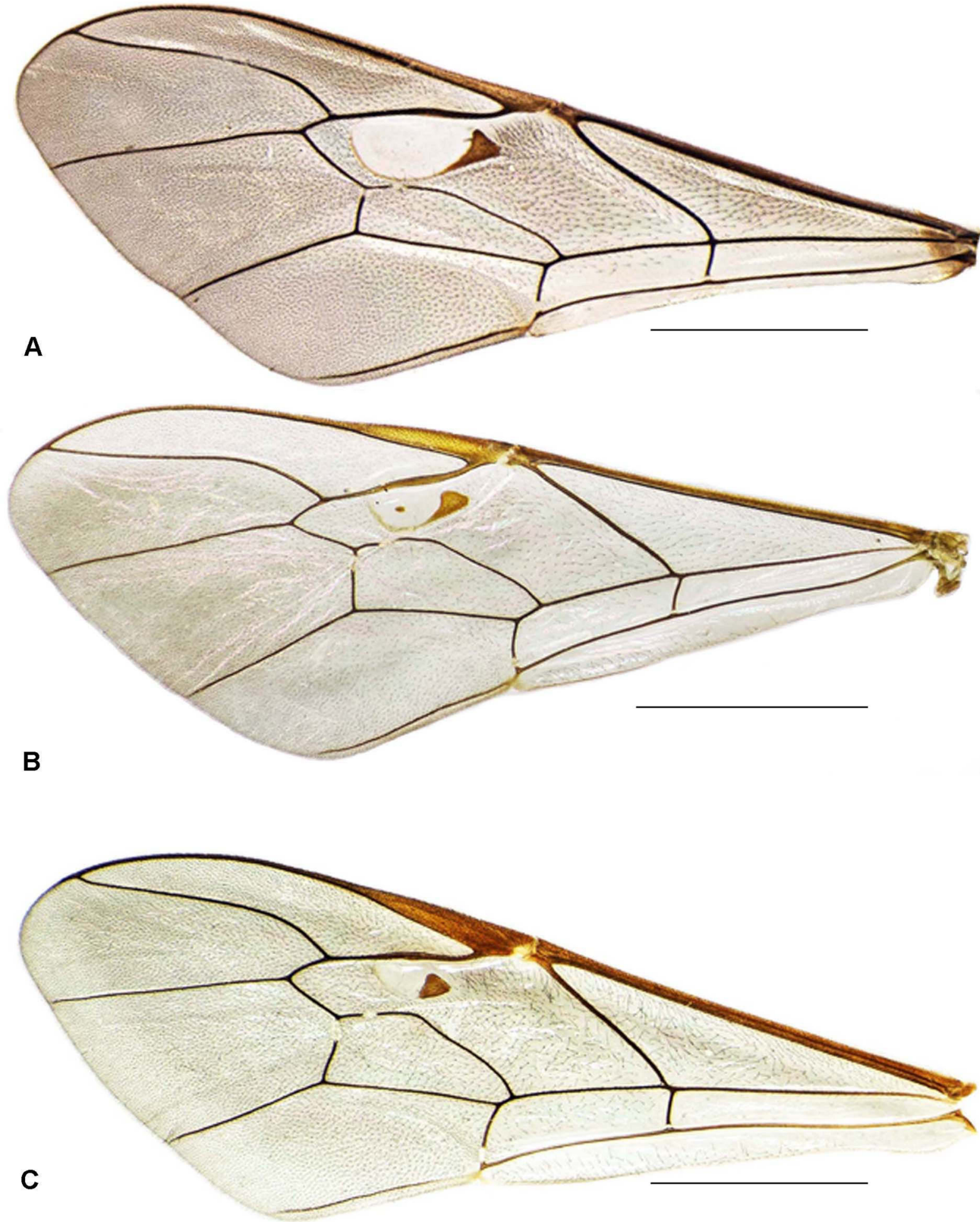

Fig. 22. Fore wing. A. Enicospilus arabicus Gadallah \& Soliman sp. nov. B. E. bicoloratus Cameron, 1912. C. E. brevicornis (Masi, 1939). Scale bars $=2.5 \mathrm{~mm}$. 

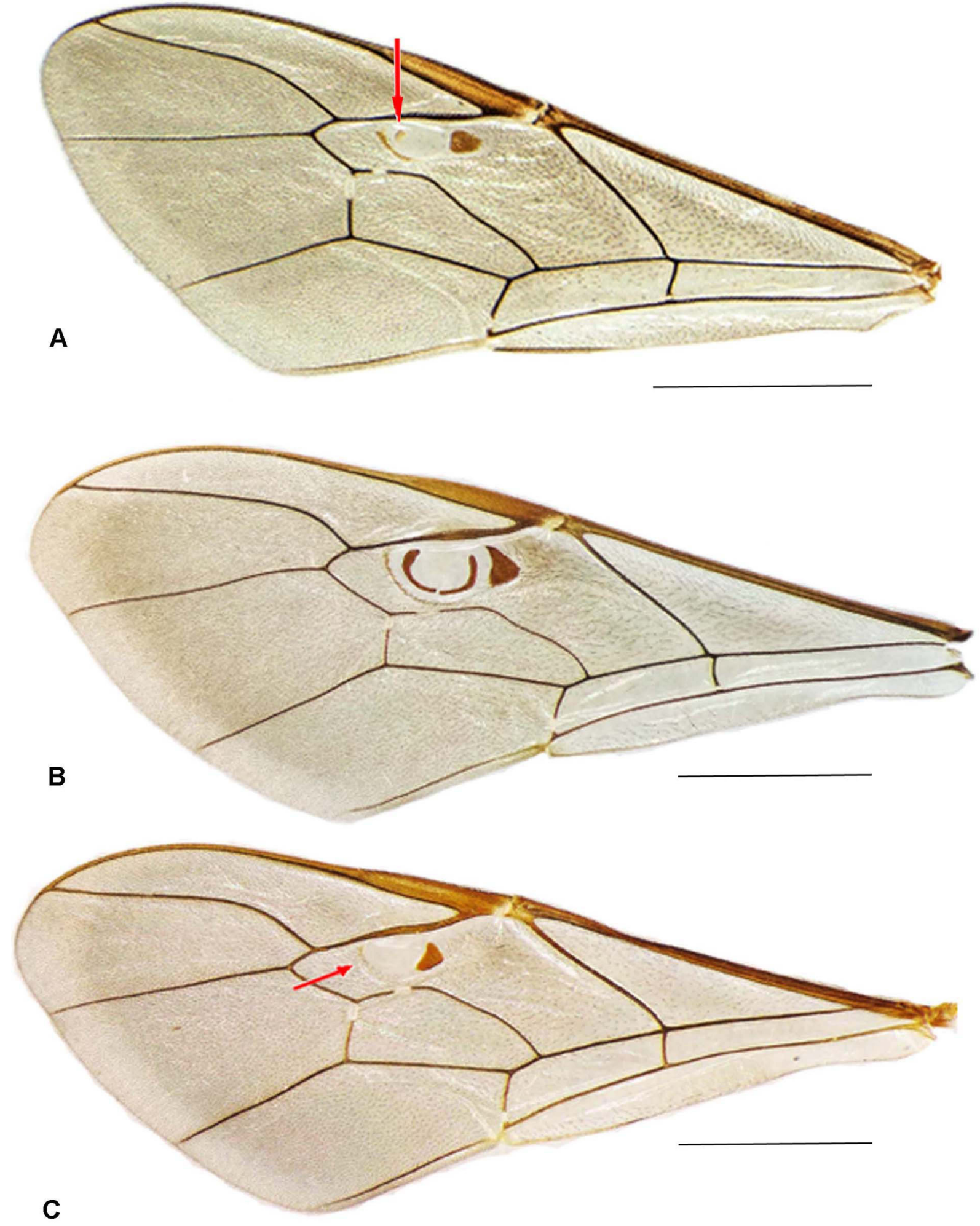

Fig. 23. Fore wing. A. Enicospilus capensis (Thunberg, 1822). B. E. divisus (Seyrig, 1935). C. E. dubius (Tosquinet, 1896). Scale bars $=2.5 \mathrm{~mm}$. 

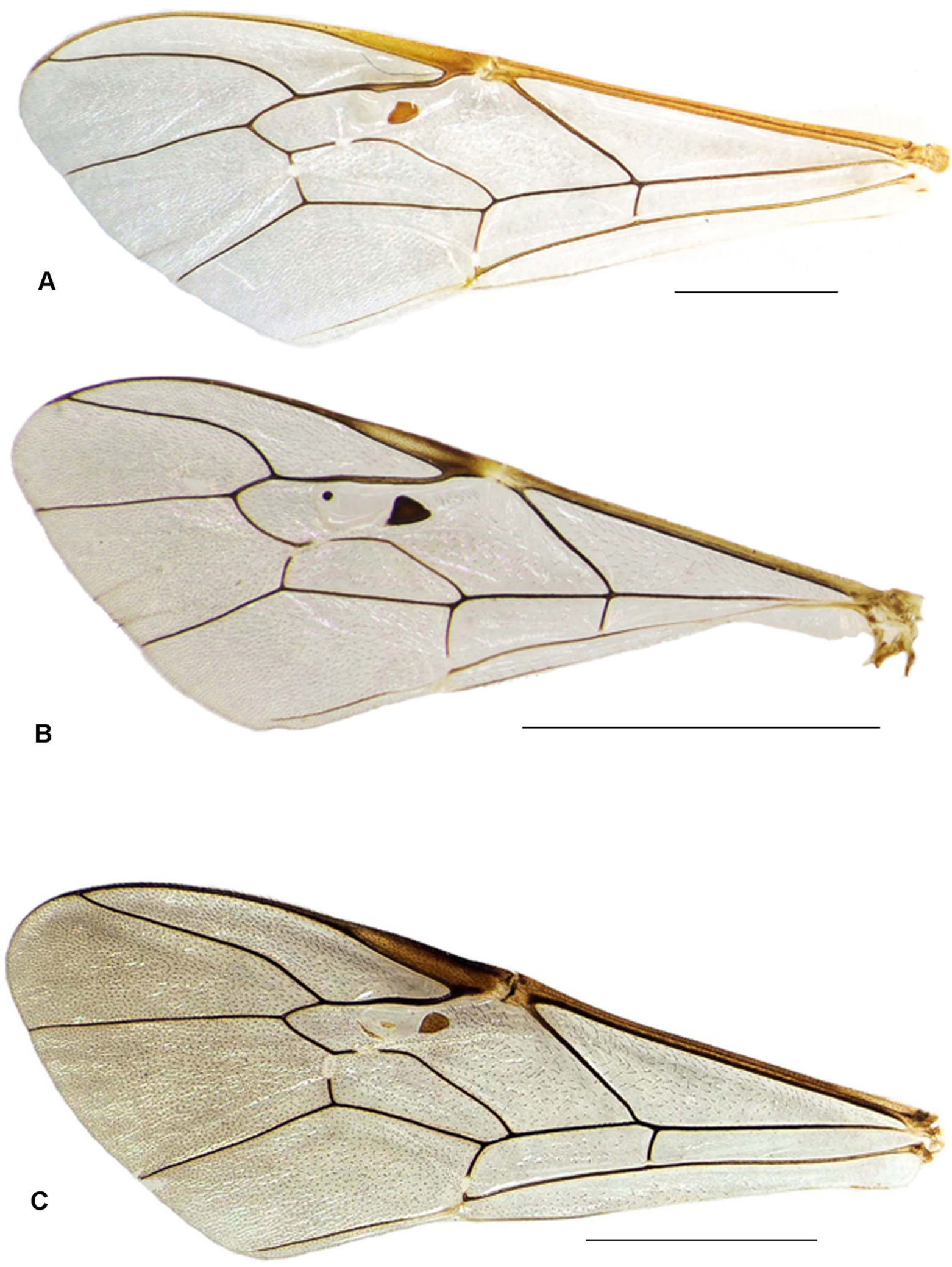

Fig. 24. Fore wing. A. Enicospilus grandiflavus Townes \& Townes, 1973. B. E. mirabilis Soliman \& Gadallah sp. nov. C. E. nervellator Aubert, 1966. Scale bars $=2.5 \mathrm{~mm}$. 

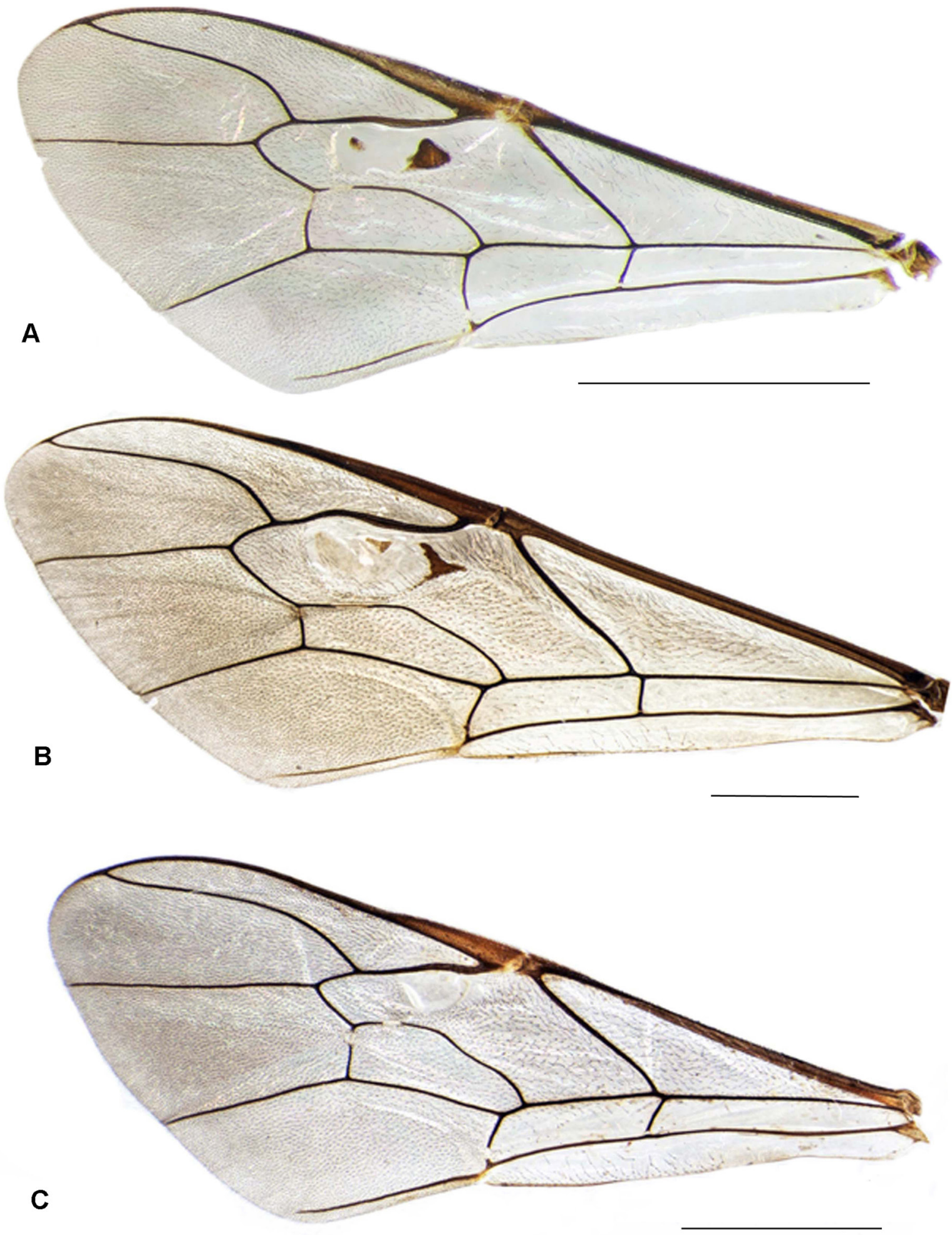

Fig. 25. Fore wing. A. Enicospilus oculator Seyrig, 1935. B. E. odax Gauld \& Mitchell, 1978. C. E. oweni Gauld \& Mitchell, 1978. Scale bars $=2.5 \mathrm{~mm}$. 

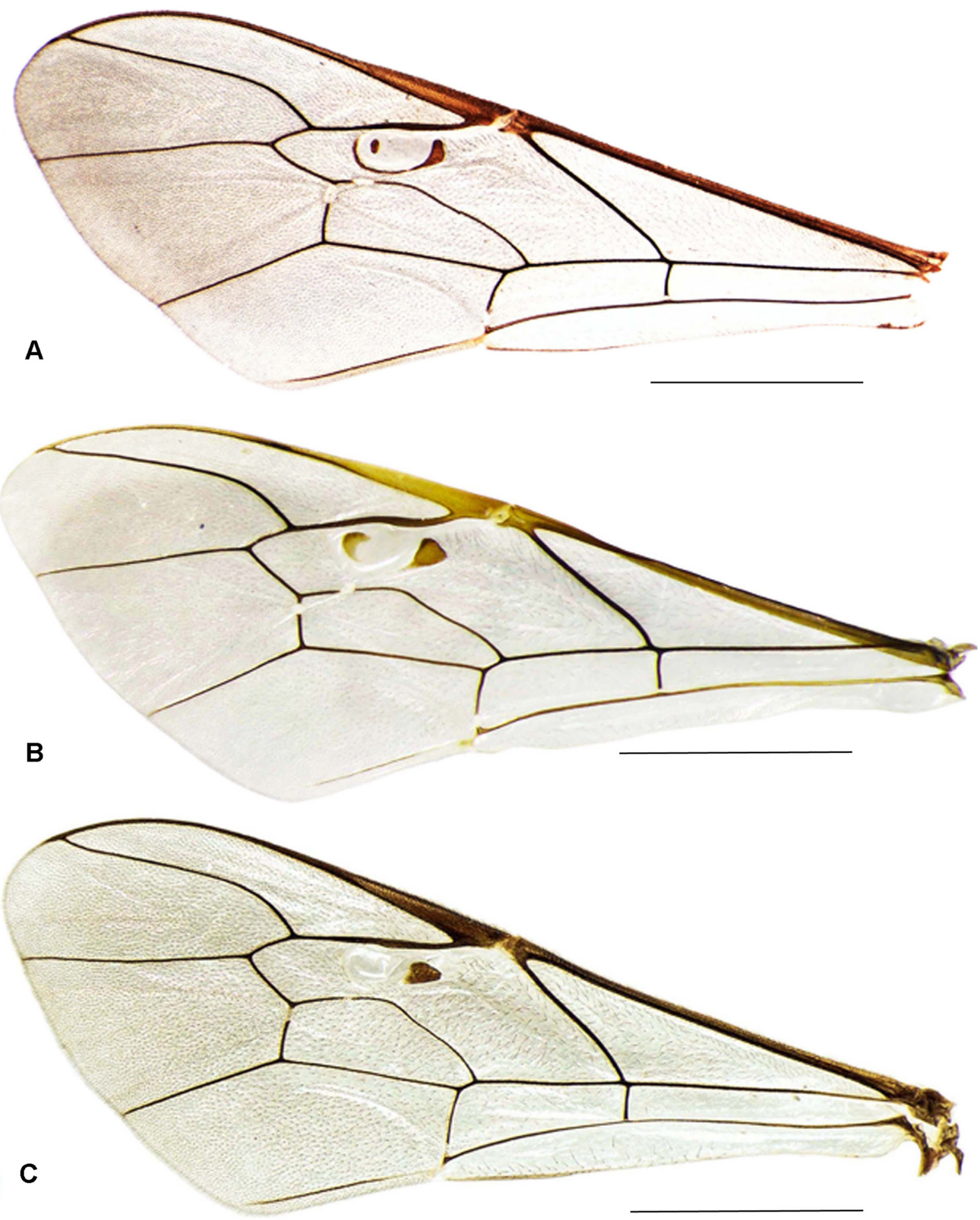

Fig. 26. Fore wing. A. Enicospilus pacificus (Holmgren, 1868). B. E. pallidus (Taschenberg, 1875). C. E. pseudoculator Gadallah \& Soliman sp. nov. Scale bars $=2.5 \mathrm{~mm}$. 

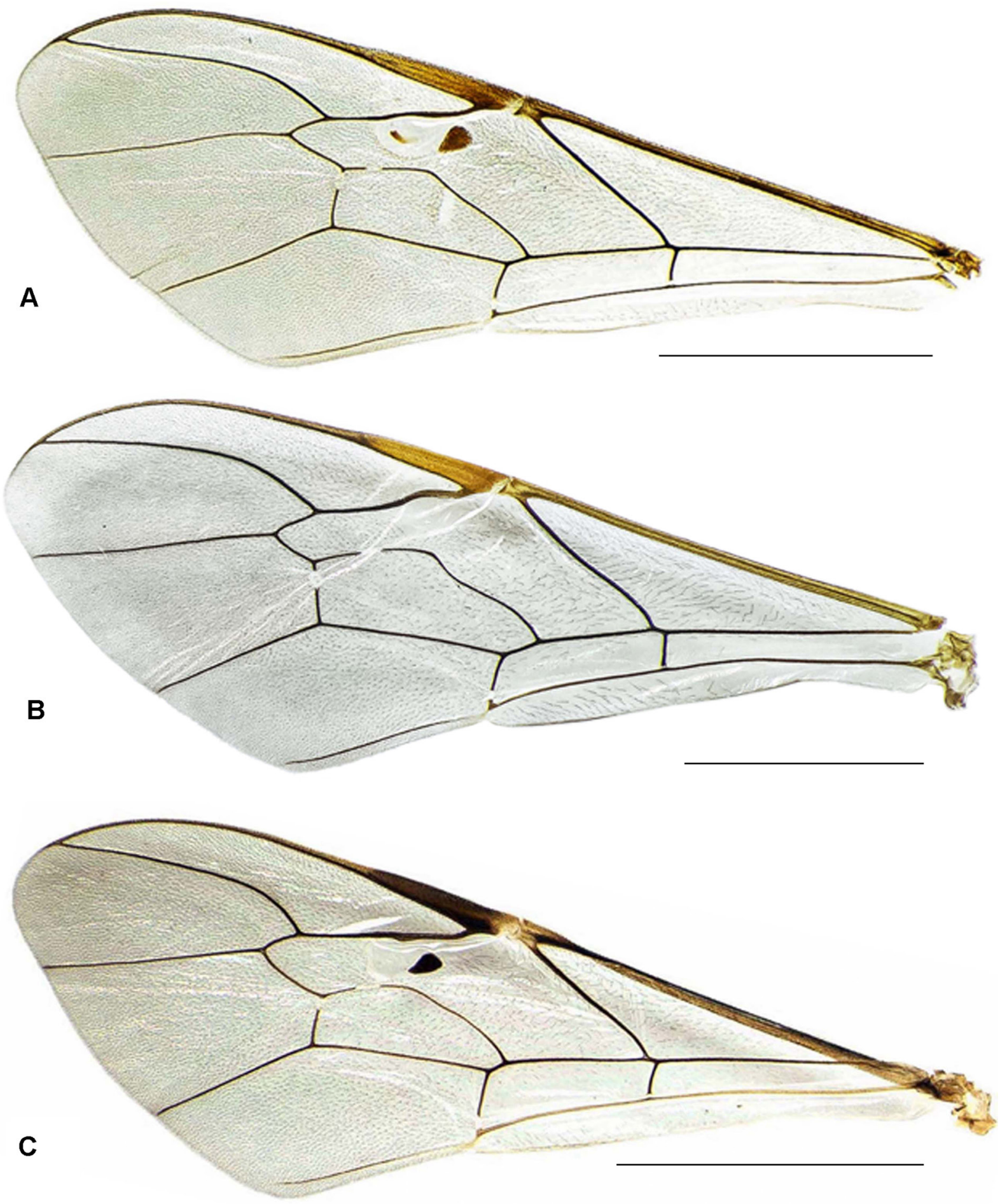

Fig. 27. Fore wing. A. Enicospilus rundiensis Bischoff, 1915. B. E. senescens (Tosquinet, 1896). C. E. shadaensis Gadallah \& Soliman sp. nov. Scale bars $=2.5 \mathrm{~mm}$. 
A
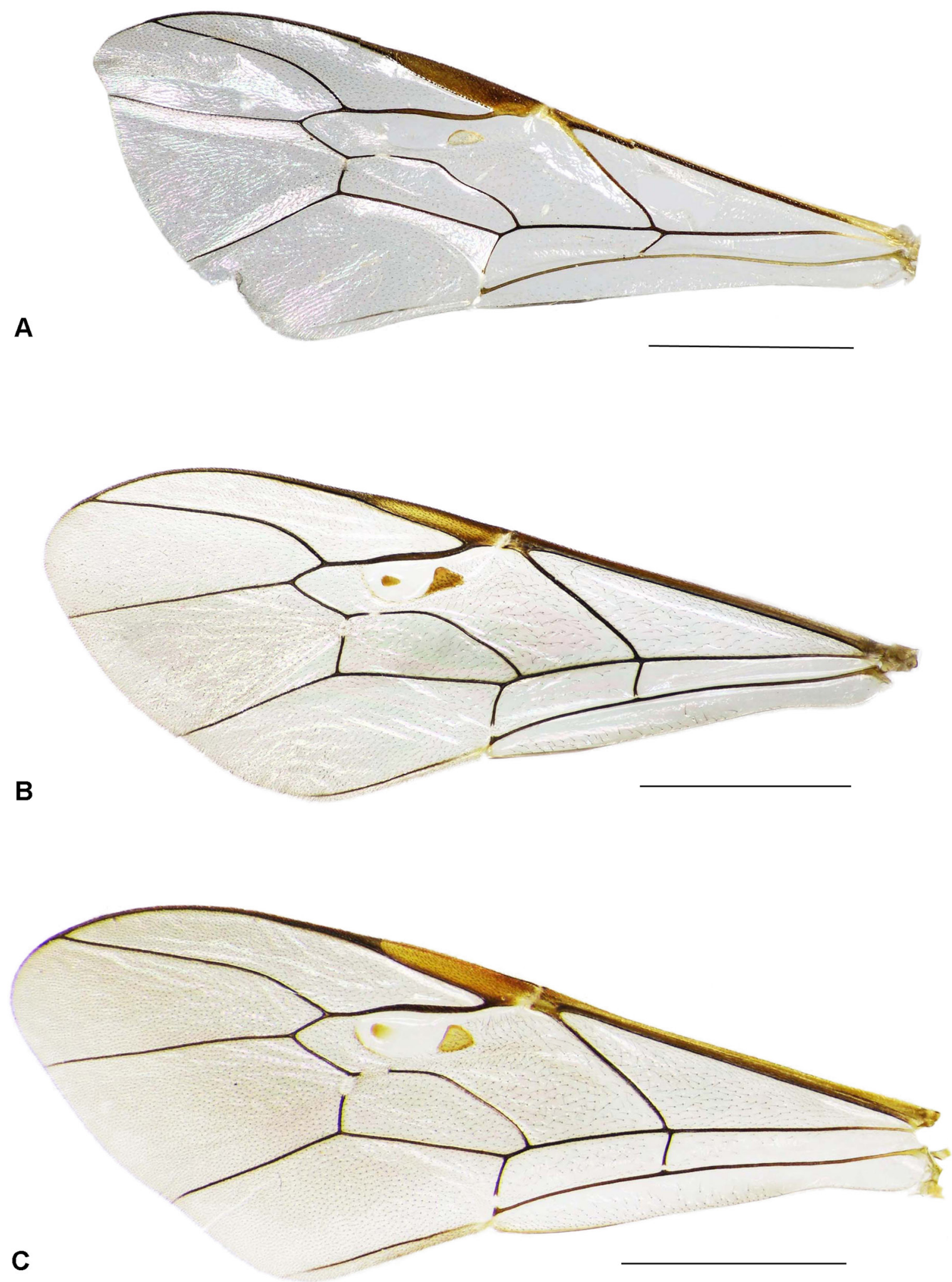

Fig. 28. Fore wing. A. Enicospilus splendidus Rousse, Soliman \& Gadallah sp. nov. B. Enicospilus sp. 2 cf. bicoloratus Cameron, 1915. C. Enicospilus sp. 1. Scale bars $=2.5 \mathrm{~mm}$. 
Enicospilus splendidus Rousse, Soliman \& Gadallah sp. nov. urn:1sid:zoobank.org:act:3ECBEFEA-22CE-4D5D-848B-B146591DBAB9

Figs 5C, 9A, 13A, 17A, 21A, 28A, 32A, 33B, 33C

\section{Diagnosis}

Moderately large sized, dark reddish brown overall, with bright yellowish to white on most of the head and mesosoma and few markings on metasoma; metasoma black posteriorly; mandible with upper tooth $1.6 \times$ as long as lower tooth; clypeus hardly convex in profile, ventral margin truncate; face subquadrate, $1.1 \times$ as high as wide; antenna short, with 52 flagellomeres; mesopleuron finely transversely striate, metapleuron with somewhat coarser striation; basal transverse carina of propodeum distinct, anterior area of propodeum finely rugose, posterior area foveolate; proximal sclerite weakly sclerotized; fore tibia with three longitudinal rows of dispersed but regularly arranged short spines along outer surface.

\section{Etymology}

From Latin, in reference to its astonishing and splendid appearance and colour.

\section{Type material}

\section{Holotype}

SAUDI ARABIA: $\partial^{\lambda}$, Riyadh, Deirab, light trap, $24^{\circ} 30.367^{\prime} \mathrm{N}, 46^{\circ} 37.166^{\prime} \mathrm{E}$, alt. 645 m, 25 Oct. 1985 , leg.? (KSMA).

\section{Description}

Male (1 specimen)

MEASUREMENTS. B 18; F 13; AI 0.6; CI 0.52; ICI 0.46; SDI 1.3; NI 1.8 ; ML 0.3; CT 1.7; OOL 0.38; POL 0.38; FI 0.5; F11-2 1.6; Fl 20 3.0.

CoLour. Dark reddish brown overall, with yellowish white on the following parts: head (except a narrow longitudinal line extending a short distance after fore ocellus to base of clypeus), clypeus, labrum and palpi, posterior margin of head around occipital carina, mandibles (except teeth ferruginous), pronotum (except a small reddish triangular area just above tegula), mesoscutum (except for three reddish brown lobes, of which middle one doesn't reach posterior margin and lateral ones do not reach anterior margin of mesoscutum), tegula, scutellum, two longitudinal oval patches on postero-lateral part of propodeum, postscutellum, subalar prominence, latero-ventral side of mesopleuron, a small area just above mesocoxa, most of metapleuron (leaving a small upper area reddish brown in colour). Metasoma darker than rest of body, with T2 and T3 above and almost all remaining tergites patterned with yellow, last sternite (subgenital plate) black; legs with coxae, trochanters and femora distinctly darker in colour than rest. Wings hyaline, fore wing with pale brown pterostigma, base of Rs $+2 r$ as well as uppermost part of Rs \& $\mathrm{M}$ and $1 \mathrm{~A}$ of hind wing pale brown to yellow, otherwise all veins are dark brown to black.

HeAD. Mandible moderately twisted, with upper tooth $1.6 \times$ as long as lower tooth, bare and without groove on outer surface; clypeus smooth, hardly convex to flat in profile, ventral margin truncate; malar space $0.3 \times$ mandibular base; face subquadrate, $1.1 \times$ as high as wide, rather smooth; gena constricted behind eyes; occipital carina complete; ocelli enlarged; antenna with 52 flagellomeres.

Mesosoma. Pronotum medio-dorsally normal, unspecialized, transversely wrinkled laterally; mesoscutum finely shagreened; epicnemial carina distinct to level of ventral corner of pronotum; postpectal carina distinct only laterally, above bases of mesocoxae, otherwise indistinct; notauli indistinct; scuto-scutellar groove smooth; scutellum nearly smooth, carinate to near apex; mesopleuron finely transversely striate, 
metapleuron with somewhat coarser striation; basal transverse carina of propodeum distinct but weak, anterior area of propodeum nearly smooth, posterior area finely foveolate.

Wings. Proximal sclerite weakly sclerotized; central sclerite totally absent; Rs $+2 r$ slightly sinuate basally; cu-a subopposite Rs \& M by about 0.25 cu-a length; hind wing with 7 distal hamuli on R1.

LEGS. Fore tibia with three longitudinal rows of regularly arranged dispersed short spines along its outer surface; hind coxa elongate; hind tarsal claw with short, widely spaced teeth; outer mid and hind tibial spurs much shorter than inner spurs $(0.23 \times$ and $0.25 \times$ respectively $)$.

Metasoma. Metasomal T2 slightly longer than T1, T2 height $0.4 \times$ its length; thyridium distinct, ellipsoid.

\section{Female}

Unknown.

\section{Distribution}

Saudi Arabia.

Enicospilus sp. 2 cf. bicoloratus Cameron, 1915

Figs 5D, 9B, 13B, 17B, 21B, 28B, 32B

\section{Diagnosis}

B 18-19; F 13; AI 0.6; ICI 0.52; CI 0.46; SDI 1.3; NI 1.8; ML 0.46; CT 1.7; POL 0.38; OOL 0.38; FI 0.5; F11-2 1.6; F120 3.0.

Body bright orange overall, with white on vertex, posterior border of eye and orbits just above emargination; metasomal T5 and T6 in profile with black ventrally, ovipositor sheath and claws black, antennae dark brown, pterostigma yellow bordered with black above and behind; mandible twisted, with upper tooth $1.25 \times$ as long as lower tooth; clypeus convex in profile, ventral margin in-turned; face $1.2 \times$ as high as wide; antenna short, with 47 flagellomeres; mesopleuron puncto-striate, metapleuron punctate; basal transverse carina of propodeum distinct but weak, anterior area finely and shallowly punctate, posterior area reticulate; proximal sclerite triangular; central sclerite fully sclerotized; hind wing with 5 distal hamuli on R1; fore tibia sparsely spinose.

\section{Material examined}

SAUDI ARABIA: 1 , , Shada Al Ala (A1 Baha), light trap 2, 27 Jan. 2015, leg. Al Dhafer et al. (KSMA).

\section{Remarks}

This species closely resembles E. bicoloratus in almost all alar indices, the puncto-striate mesopleuron, the punctate metapleuron and the black apex of the metasoma. However, it differs from E. bicoloratus in the following: lower face $1.2 \times$ as long as broad (in E. bicoloratus $1.4 \times$ as long as broad); antennae relatively short, with 47 flagellomeres (in E. bicoloratus with 50-56 flagellomeres); hind wing with 5 distal hamuli on R1 (in E. bicoloratus with 5-7 distal hamuli on R1).

\section{Distribution}

Saudi Arabia. 

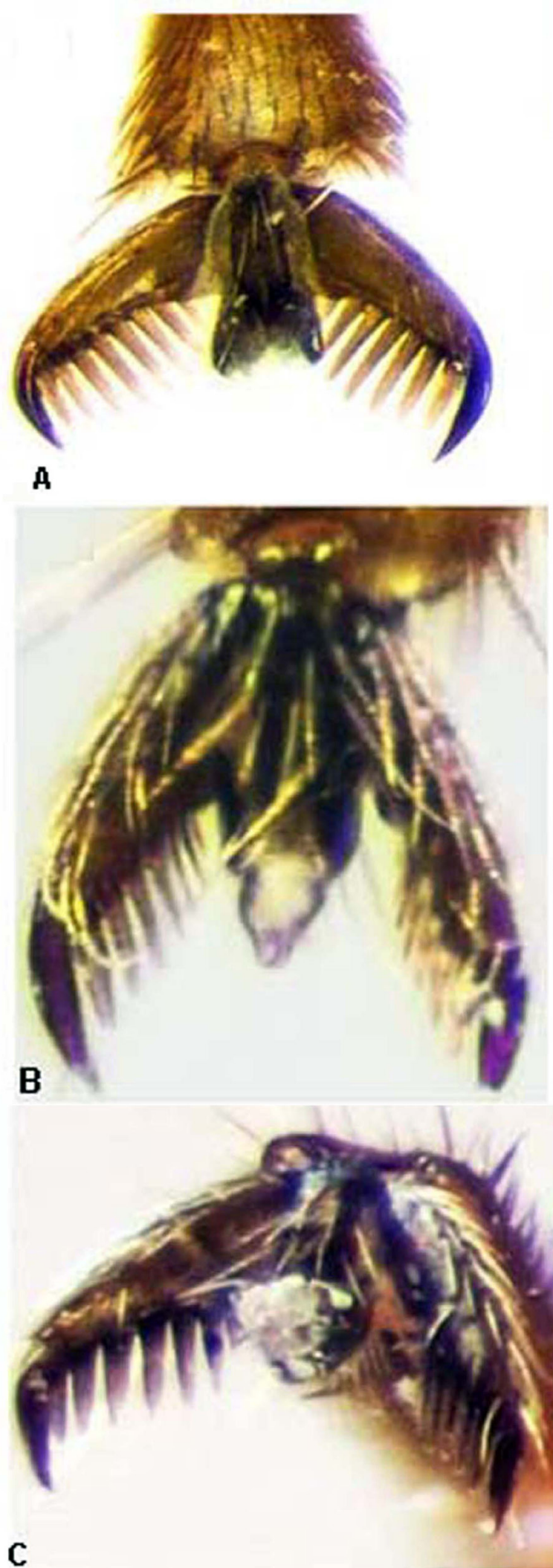
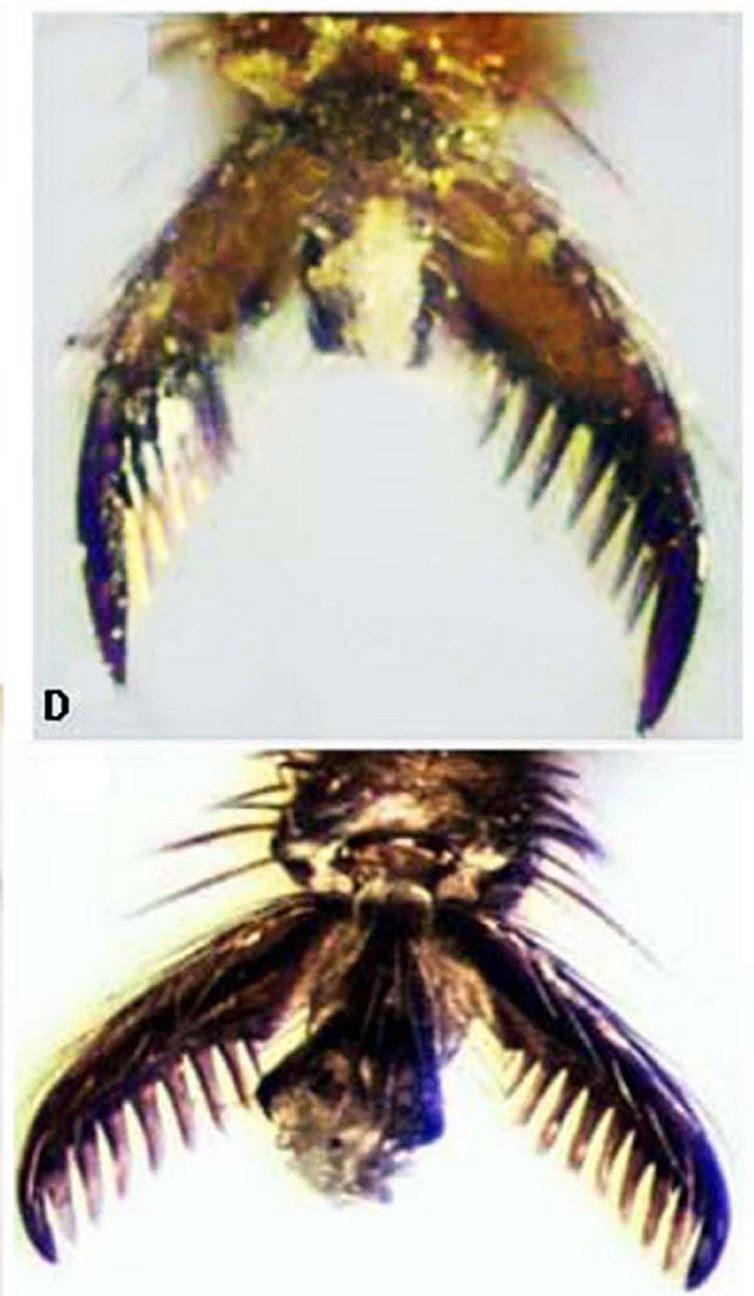

E

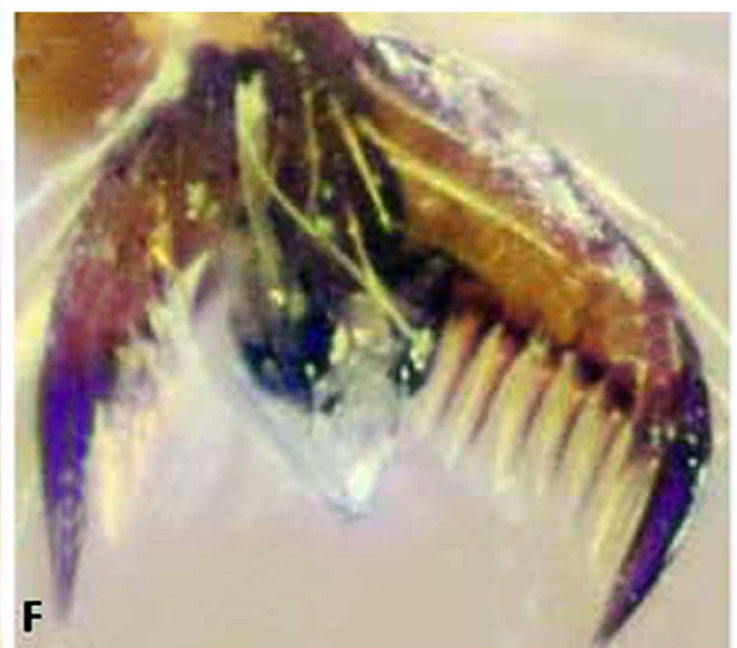

Fig. 29. Hind tarsal claws. A. Enicospilus arabicus Gadallah \& Soliman sp. nov. B. E. bicoloratus Cameron, 1912. C. E. brevicornis (Masi, 1939). D. E. capensis (Thunberg, 1822). E. E. divisus (Seyrig, 1935). F. E. dubius (Tosquinet, 1896). 

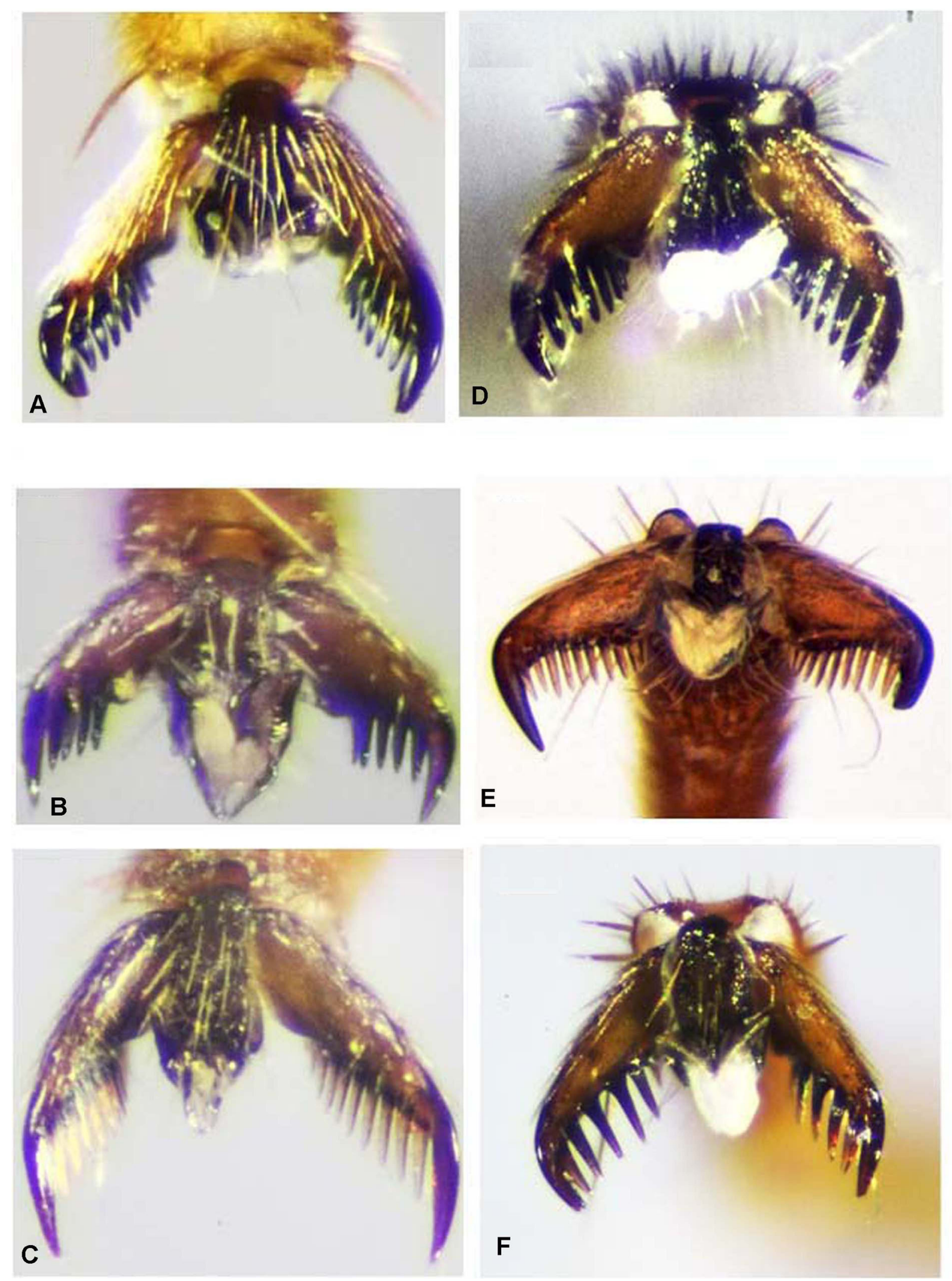

Fig. 30. Hind tarsal claw. A. Enicospilus grandiflavus Townes \& Townes, 1973. B. E. mirabilis Soliman \& Gadallah sp. nov. C. E. nervellator Aubert, 1966. D. E. oculator Seyrig, 1935. E. E. odax Gauld \& Mitchell, 1978. F. E. oweni Gauld \& Mitchell, 1978. 

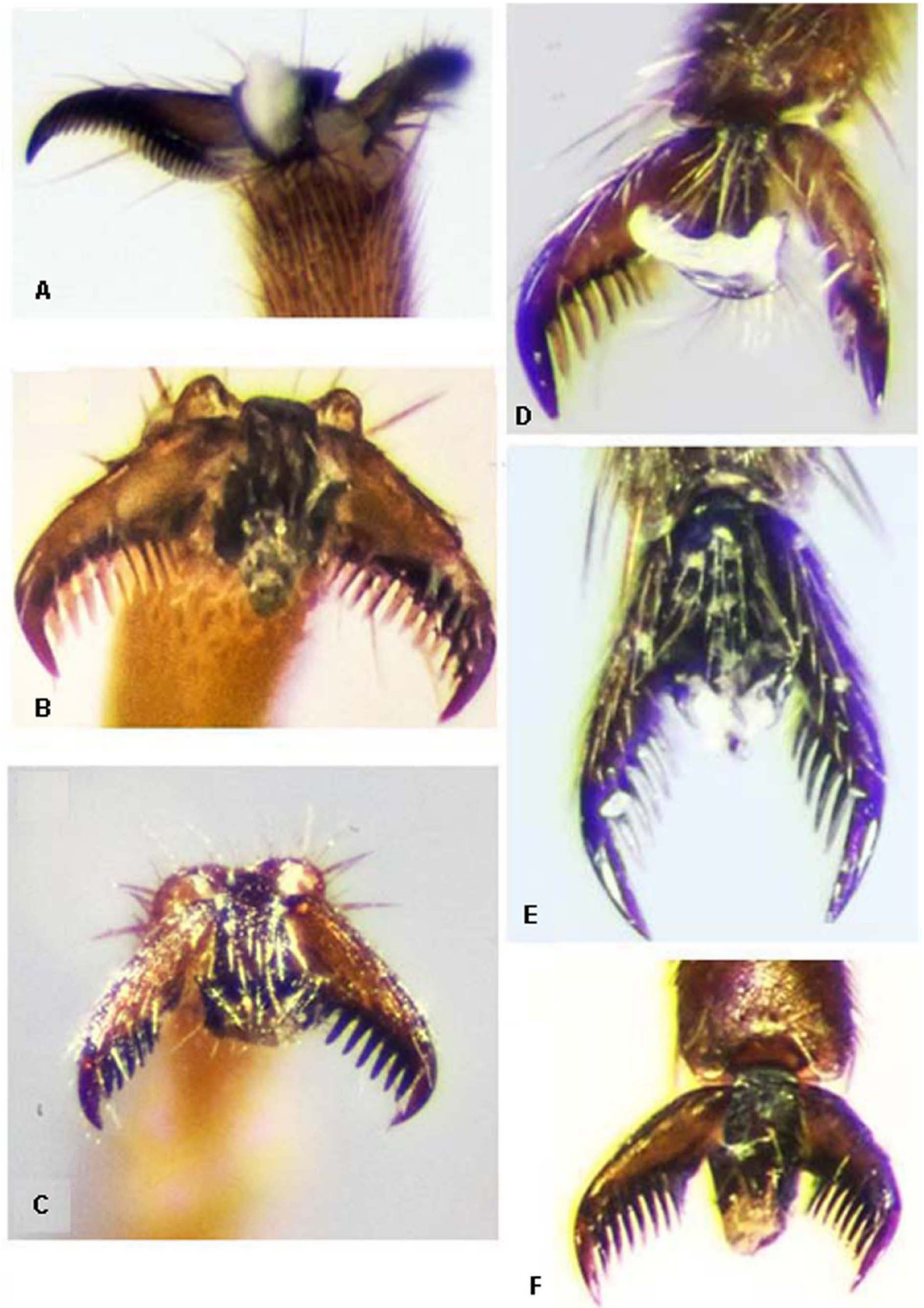

Fig. 31. Hind tarsal claw. A. Enicospilus pacificus (Holmgren, 1868). B. E. pallidus (Taschenberg, 1875). C. E. pseudoculator Gadallah \& Soliman sp. nov. D. E. rundiensis Bischoff, 1915. E. E. senescens (Tosquinet, 1896). F. E. shadaensis Gadallah \& Soliman sp. nov. 

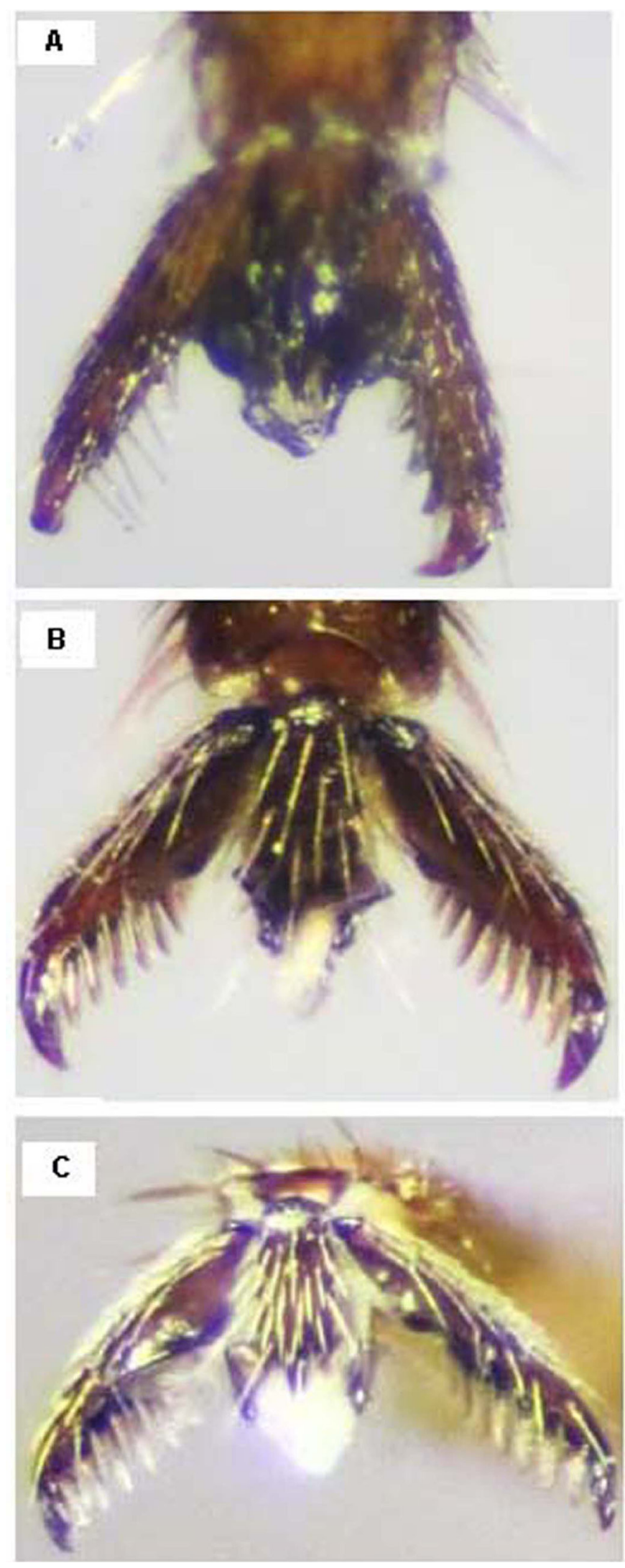

Fig. 32. Hind tarsal claw. A. Enicospilus splendidus Rousse, Soliman \& Gadallah sp. nov. B. Enicospilus sp. 2 cf. bicoloratus Cameron, 1915. C. Enicospilus sp. 1 
Enicospilus sp. 1

Figs 5E, 9C, 13C, 17C, 21C, 28C, 32C

\section{Diagnosis}

B 18-20; F 13; AI 0.2; ICI 0.55; CI 0.4; SDI 1.2; NI 1.7; ML 0.4; CT 2.0; POL 0.6; OOL 0.1; FI 0.5; F11-2 1.5; F120 2.0.

Body reddish brown overall, with pale yellow on posterior border of eye, vertex and orbits (just above eye emargination), pterostigma of fore wing yellow, all wing veins black; mandible twisted, upper tooth distinctly $2.0 \times$ as long as lower tooth, outer surface fringed with fine setae; clypeus convex in profile, ventral margin in-turned; face subquadrate, $1.1 \times$ as high as wide, finely punctate, with spaces between punctures equal to puncture diameter or slightly more; occipital carina complete; gena constricted behind eye; ocelli enlarged; antenna with 52 flagellomeres; pronotum simple, unspecialized; mesopleuron densely finely punctate, somewhat coarser on metapleuron; epicnemial carina weak; postpectal carina complete; notauli indistinct; basal transverse carina of propodeum distinct, anterior area superficially finely punctate, posterior area areolate; proximal sclerite well sclerotized, central sclerite weakly sclerotized proximally; hind wing with 7 distal hamuli on R1; metasoma densely covered with pale fine and short setae.

\section{Material examined}

SAUDI ARABIA: 1 q, Raydah (Asir), light trap, 6 Jun. 2014, leg. Al Dhafer et al. (KSMA).

\section{Remarks}

This species is characterized by its dark colour and very strongly convex clypeus, which are uncommon features. Comparing with other Afrotropical species with an ill-defined central sclerite gives no results, meaning that it could be either an Oriental or Mediterranean species, so we add it here as Enicospilus sp. awaiting collection of further specimens.

\section{Distribution}

Saudi Arabia.

\section{Discussion}

The first general observation emerging from the present study is the strong correlation of the Enicospilus fauna with the intermediate biogeographical situation of Saudi Arabia. Of the 16 species collected in southwestern Saudi Arabia for which biogeographical data are available, almost all are exclusively Afrotropical, the only exception being the widely distributed E. capensis (Table 1). Conversely, the four species collected in Riyadh are Afrotropical, Palaearctic or widely distributed. This is closely correlated with the floristic composition of this area as has been reported by several authors (e.g., Eig 1938; Bolton 1994; Aldawood et al. 2011; Sharaf \& Aldawood 2011, 2012; Sharaf et al. 2012a, 2012b, 2014; El-Hawagry et al. 2013, 2015).

A second observation of ecological significance is provided by the large proportion of Enicospilus species in the local fauna with rather short antennae and/or profuse ivory markings. Only 10 out of 23 species (43\%) have antennae which may reach more than 55 flagellomeres, while this proportion is $75 \%$ in the entire Afrotropical fauna (Gauld \& Mitchell 1978; Yu et al. 2012). Moreover, in the five species described here and probably endemic to the Arabian Peninsula, only E. arabicus sp. nov. barely reaches beyond this threshold. Furthermore, five of the 23 species exhibit ivory markings at least on the mesosoma, and this includes all newly described species with the exception again of $E$. arabicus sp. nov. Among the approximately 150 species of the Afrotropical fauna, only four have such conspicuous pale 
markings. This is not surprising, since short antennae and pale markings are common features in species of arid open areas (Gauld \& Mitchell 1978, 1981). This light pattern is probably related to light reflection and temperature refraction.

However, because of the biodiversity richness of Saudi Arabia, more and more species of this genus are expected to occur. Therefore, further collections and studies are needed to clarify the distribution of this genus in other parts of this large country.
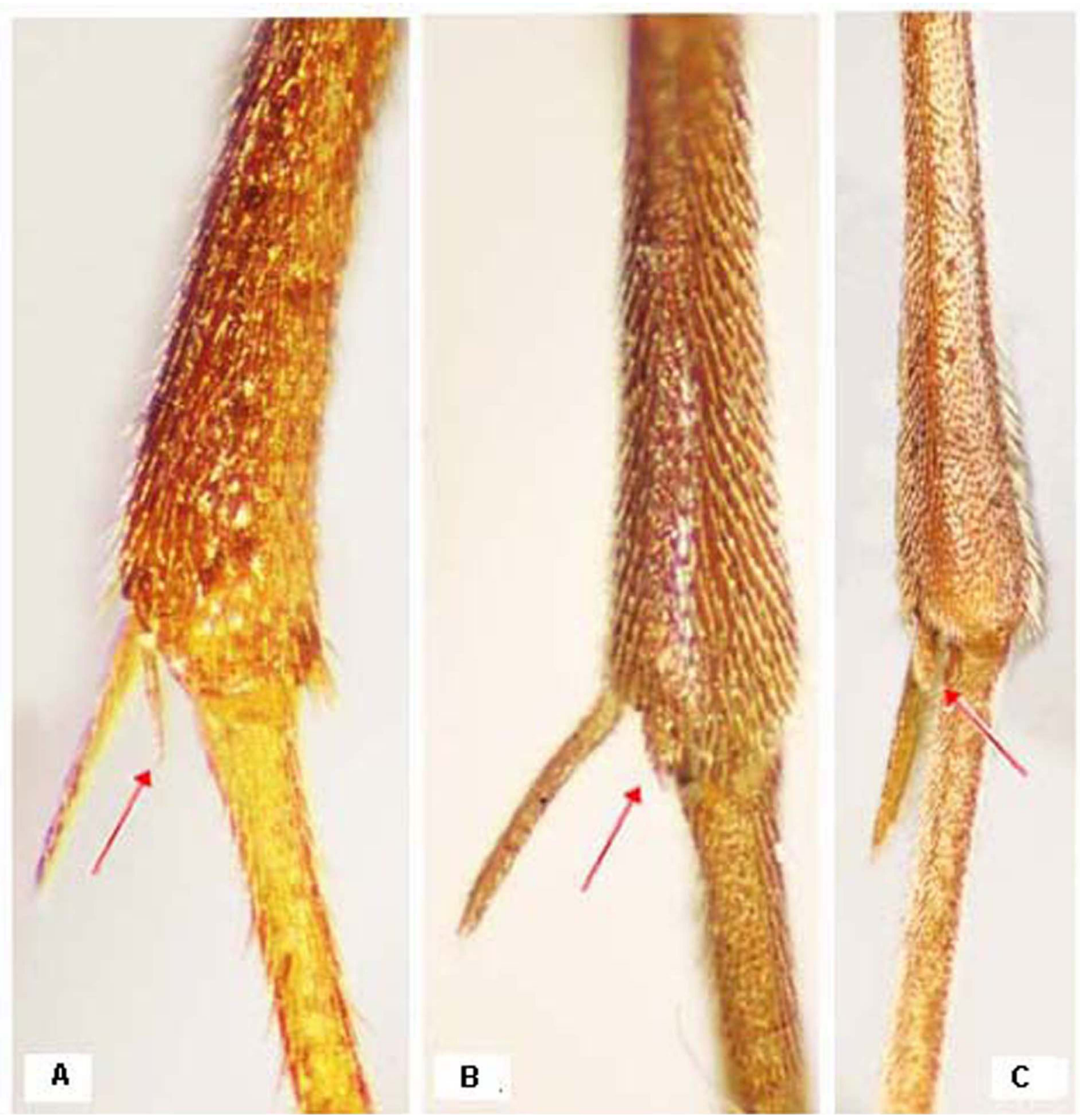

Fig. 33. A. Mid tibial spurs of Enicospilus nervellator Aubert, 1966. B. Mid tibial spurs of E. splendidus Rousse, Soliman \& Gadallah sp. nov. C. Hind tibial spurs of E. splendidus Rousse, Soliman \& Gadallah sp. nov. 


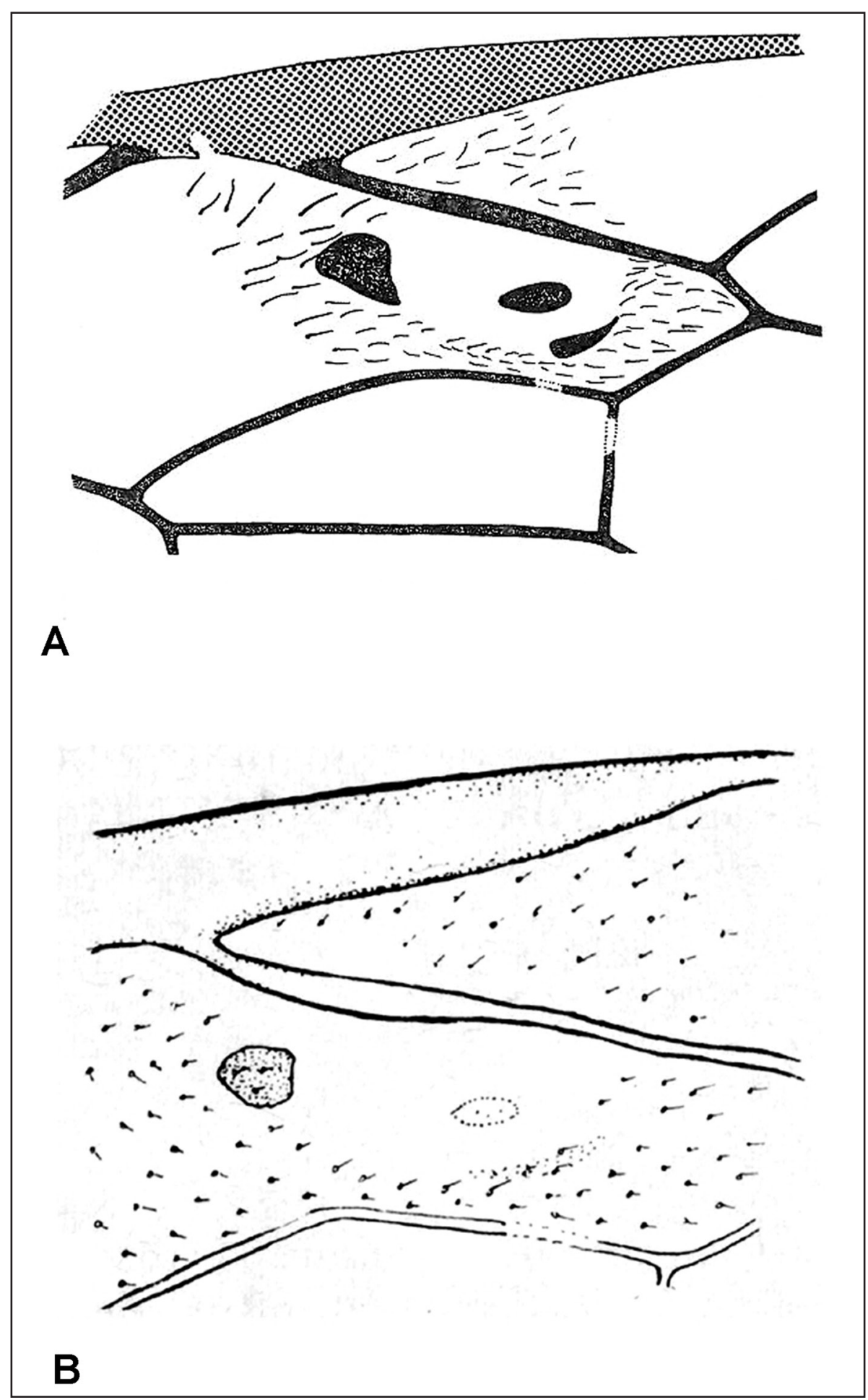

Fig. 34. Disco-submarginal cell. A. Enicospilus psammus (after Gauld \& Mitchell 1978). B. E. perlatus (after Shestakov 1926). 


\section{Acknowledgements}

The material collected for the present study and the photographic work were supported by the Deanship of Scientific Research of King Saud University for the research group NO (RGP-1437-009). Gratitude is expressed to all the members of the King Saud University Museum of Arthropods (KSMA), Plant Protection Department, College of Food and Agriculture Sciences, King Saud University, for help in collecting the specimens. Sincere gratitude is offered to Claire Villemant and Eric Guilbert (MNHN/ UMR7205 ISYEB) for the use of the BoEM and SSM platforms for molecular analyses and sequencing. Sincere thanks are also due to Gavin Broad (Natural History Museum, London) for his kind hospitality during the visit of the first author to the Natural History Museum of London, and for his kind help in confirming the identification of some species. We are also grateful to Adrian Pont (Oxford University Museum of Natural History, UK) and Andrew Polaszek (Natural History Museum, London) for kindly revising sections of the manuscript linguistically and for their support with some taxonomic problems.

\section{References}

Aldawood A.S., Sharaf M.R. \& Taylor B. 2011. First record of the Myrmicine ant genus Carebara Westwood, 1840 (Hymenoptera: Formicidae) from Saudi Arabia with description of a new species C. abuhurayri sp.n. ZooKeys 92: 61-69. https://doi.org/10.3897/zookeys.92.770

Aldhebiani A.Y. \& Howladar S.M. 2013. Floristic diversity and environmental relations in two valleys, South West Saudi Arabia. International Journal of Science and Research 4 (2): 1916-1925.

Al Mobdel K.S. 2001. Nature Preserves in Riyadh Region. Al Mobdel Publisher, Riyadh.

Aubert J.F. 1966. Description de dix espèces nouvelles s'ajoutant aux Ichneumonides de France et du Bassin mediterraneen. Bulletin de la Societé Entomologique de Mulhouse 1966: 37-46.

Aubert J.F., Halperin J. \& Gerling D. 1984. Les Ichneumonides d'Israel. Entomophaga 29 (2): 211-235. https://doi.org/10.1007/BF02372110

Barahoei H., Rakhshani E. \& Riedel M. 2012. A checklist of Ichneumonidae (Hymenoptera: Ichneumonoidea) from Iran. Iranian Journal of Animal Biosystematics 8 (2): 83-132.

Bischoff H. 1915. Zoologische Ergebnisse der Professor Hans Meyerschen Expedition nach Ostafrika 119. Mitteilungen aus dem Zoologischen Museum in Berlin 7 (3): 471-477.

Bolton B. 1994. Identification Guide to the Ant Genera of the World. Harvard University Press, Cambridge (MA).

Bordera S., Selfa J. \& Jimenéz R. 1987. Contribución al conocimiento del género Enicospilus Stephens, 1835 (Hym. Ichneumonidae) en España. Boletin de la Asociación Española de Entomologia 11: 221233. http://hdl.handle.net/10045/19301

Broad G.R. \& Shaw M.R. 2016. The British species of Enicospilus (Hymenoptera: Ichneumonoidea: Ophioninae). European Journal of Taxonomy 187: 1-31. https://doi.org/10.5852/ejt.2016.187

Cameron P. 1906. Descriptions of new species of parasitic Hymenoptera chiefly in the collection of the South African Museum, Cape Town. Annals of the South African Museum 5: 17-186.

Cameron P. 1912. On the Hymenoptera from Belgian Congo in the Congo Museum, Tervueren. Annales de la Société Entomologique de Belgique 56: 357-401.

Available from http://biodiversitylibrary.org/page/12848247 [accessed 3 Oct. 2017].

Eig A. 1938. Taxonomic studies on the Oriental species of the genus Anthemis. Palestine Journal of Botany, Jerusalem 1: 161-224.

El-Hawagry M.S., Khalil M.W., Sharaf M.R., Fadl H.H. \& Aldawood A.S. 2013. A preliminary study on the insect fauna of Al-Baha Province, Saudi Arabia, with descriptions of two new species. ZooKeys 274: 1-88. https://doi.org/10.3897/zookeys.274.4529 
El-Hawagry M.S., Sharaf M.R., Al Dhafer H.M., Fadl H.H. \& Aldawood A.S. 2015. Addenda to the insect fauna of Al-Baha Province, Kingdom of Saudi Arabia with zoogeographical notes. Journal of Natural History 50 (19-20): 1209-1236. https://doi.org/10.1080/00222933.2015.1103913

El-Moursy A., El-Hawagry M., Abdeldayem M. \& Fadl H.H. 2001. Insect diversity in Zaranik Protectorate, Northern Sinai, Egypt. Egyptian Journal of Natural History 3: 62-80.

Enderlein G. 1914. Hymenoptera IV: Ichneumonidae. In: Michaelsen W. (ed.) Beiträge Zur Kenntnis Der Land-Und Süsswasserfauna Deutsch-Südwestafrikas: 11-233. Band 1 Hamburg, Germany.

Enderlein G. 1918. Ichneumonidae In: Michaelson W. (ed.) Beiträge zur Kenntnis der Land- und Süsswasserfauna Deutsch Sudwestafrikas 2 (4): 22.

Enderlein G. 1921. Beiträge zur aussereuropäischer Ichneumoniden 5. Über die Familie Ophionidae. Entomological Society of Stettin 82: 1-45.

Gauld I.D. 1985. The phylogeny, classification and evolution of parasitic wasps of the subfamily Ophioninae (Ichneumonidae). Bulletin of the British Museum (Natural History), Entomology Series 51 (2): 61-185.

Gauld I.D. 1988. A survey of the Ophioninae (Hymenoptera: Ichneumonidae) of tropical Mesoamerica with special reference to the fauna of Costa Rica. Bulletin of the British Museum (Natural History), Entomology Series 57: 1-309. Available from http://biodiversitylibrary.org/page/41080103 [accessed 3 Oct. 2017].

Gauld I.D. \& Huddleston T. 1976. The nocturnal Ichneumonoidea of the British Isles, including a key to the genera. Entomologist's Gazette 27: 35-49.

Gauld I.D. \& Mitchell P.A. 1978. The Taxonomy, Distribution and Host Preferences of African Parasitic Wasps of the Subfamily Ophioninae. CAB: Slough. Commonwealth Institute of Entomology, London.

Gauld I.D. \& Mitchell P.A. 1981. The Taxonomy, Distribution and Host Preferences of Indo-Papuan Parasitic Wasps of the Subfamily Ophioninae. CAB: Slough. Commonwealth Institute of Entomology, London.

Ghazanfar S.A. \& Fischer M. 1998. Vegetation of the Arabian Peninsula. Geobotany 25: 1-362.

Gracía A.G. 2011. Tres species nueves de Enicospilus (Ichneumonidae: Ophioninae) de Colombia. Revista Colombiana de Entomología 37 (1): 145-151.

Harris R.A. 1979. A glossary of surface sculpturing. Occasional Papers in Entomology 28: 1-31.

Hedwig K. von. 1957. Ichneumoniden und Braconiden aus Iran 1954 (Hymenoptera). Jahresheft des vereins für Vaterländische Naturkunde 112 (1): 103-117.

Holmgren A.E. 1868. Hymenoptera. Species novas descripsit. Kongliga Svenska Fregatten Eugenies Resa omkring jorden, Zoologi 6: 391-442.

Hölzel H. 1998. Zoogeographical features of the Arabian Peninsula. Acta Zoologica Fennica 209: 129140.

Horstmann K. 1981. Insects of Saudi Arabia. Hymenoptera: Fam. Ichneumonidae. Fauna of Saudi Arabia 3: 425-434.

Kolarov J. \& Ghahari H. 2005. A catalogue of Ichneumonidae (Hymenoptera) from Iran. Linzer biologische Beiträge 37 (1): 503-532.

Kriechbaumer J. 1901. Bemerkungen über Ophioniden (Hym.). Zeitschrift für systematische Hymenopterologie und Dipterologie 1: 152-155.

Madl M. 1996. Ephialtinae und Ophioninae von der Insel Sainte Marie (Madagaskar) (Hymenoptera; Ichneumonidae). Linzer biologische Beiträge 28 (2): 835-838. 
Masi L. 1939. Hymenoptera, Chalcididae, Cynipidae, Ichneumonidae, Braconidae, Bethylidae. Missione Biologica nel Paese dei Borana 3 (2): 21-44.

Morley C. 1912. A revision of the Ichneumonidae based on the collection in the British Museum (Natural History) with descriptions of new genera and species Part I. Tribes Ophionides and Metopiides. British Museum, London. https://doi.org/10.5962/bhl.title.8761

Ratnasingham S. \& Hebert P.D.N. 2007. BOLD: The Barcode of Life data system. Molecular Ecology Notes 7: 335-364.

Roman A. 1924. Zoological results of the Swedish Expedition to Central-Afrika 1921. Insecta: Hymenoptera, Entomophaga. Arkiv för Zoologi 16 (17): 1-9.

Rousse P. \& van Noort S. 2014. Afrotropical Ophioninae (Hymenoptera, Ichneumonidae): an update of Gauld and Mitchell's revision, including two new species and an interactive matrix identification key. ZooKeys 456: 59-73. https://doi.org/10.3897/zookeys.456.8140

Rousse P. \& Villemant C. 2012. Ichneumons in Reunion Island: a catalogue of the local Ichneumonidae (Hymenoptera) species, including 15 new taxa and a key to species. Zootaxa 3278: 1-57. https://doi.org/10.15468/iiiien

Rousse P., Quicke D.L.J., Matthee C.A., Lefeuvre P. \& van Noort S. 2016. A molecular and morphological reassessment of the phylogeny of the subfamily Ophioninae (Hymenoptera: Ichneumonidae). Biological Journal of the Linnean Society 178 (1): 128-148. https://doi.org/10.1111/zoj.12405

Saussure H. de. 1892. In: Grandidier A. (ed.) Histoire physique, naturelle et politique de Madagascar. Vol. 20: Histoire naturelle des Hyménoptères. L'Imprimerie Nationale, Paris. Available from http://biodiversitylibrary.org/page/39794875 [accessed 3 Oct. 2017].

Seyrig A. 1935. Mission scientifique de l'Omo. Tome III. Fascicule 18. Hymenoptera, II. Ichneumonidae: Cryptinae, Pimplinae, Tryphoninae et Ophioninae. Mémoires du Muséum national d'Histoire naturelle 4: $1-100$.

Sharaf M.R. \& Aldawood A.S. 2011. Monomorium dryhimi sp. n., a new ant species (Hymenoptera: Formicidae) of the M. monomorium group from Saudi Arabia, with a revised key to the Arabian Monomorium monomorium-group. ZooKeys 106: 47-54. https://doi.org/10.3897/zookeys.106.1390

Sharaf M.R. \& Aldawood A.S. 2012. A new ant species of the genus Tetramorium Mayr, 1855 (Hymenoptera: Formicidae) from Saudi Arabia, including a revised key to the Arabian species. PLOS ONE 7 (2): e30811. https://doi.org/10.1371/journal.pone.0030811

Sharaf M.R., Aldawood A.S. \& El-Hawagry M.S. 2012a. A new ant species of the genus Tapinoma (Hymenoptera: Formicidae) from Saudi Arabia with a key to the Arabian species. ZooKeys 212: 35-43. https://doi.org/10.3897/zookeys.212.3325

Sharaf M.R., Aldawood A.S. \& El-Hawagry M.S. 2012b. First record of the ant subfamily Aenictinae (Hymenoptera: Formicidae) from Saudi Arabia, with the description of a new species. ZooKeys 228: 39-49. https://doi.org/10.3897/zookeys.228.3559.

Sharaf M.R., Fischer B.L. \& Aldawood A.S. 2014. First record of the Myrmicine ant genus Meranoplus Smith, 1853 (Hymenoptera: Formicidae) from the Arabian Peninsula with description of a new species and notes on the zoogeography of the southwestern Kingdom of Saudi Arabia. PLOS ONE 9: e111298. https://doi.org/10.1371/journal.pone.0111298

Shaumar N. 1966. Les Ichneumonides d'Egypte. Entomophaga 11: 441-469. https://doi.org/10.1007/BF02390698

Shestakov A. 1926. Tabula diagnostic et species novae palaearcticae generis Enicospilus Stephen. Konowia 5: 25-32. 
Stephens J.F. 1829. A Systematic Catalogue of British Insects: Being an Attempt to Arrange all the Hitherto Discovered Indigenous Insects in Accordance with their Natural Affinities. Containing also the References to Every English Writer on Entomology, and to the Principal Foreign Authors. With all the Published British Genera to the Present Time. Vol. 1. Baldwin \& Cradock, London.

Stephens J.F. 1835. Illustrations of British Entomology. Vol. 7: Mandibulata. Baldwin \& Cradock, London.

Szépligeti G. 1905. Hymenoptera. Ichneumonidae (Gruppe Ophionoidea), subfam. PharsaliinaePorizontinae. Genera Insectorum 34: 1-68.

Szépligeti G. 1906. Neue exotische Ichneumoniden aus der Sammlung des Ungarischen NationalMuseums. Annales historico-naturales Musei nationalis hungarici 4: 119-159.

Taschenberg E. 1875. Zur Kenntnis der Gattung Ophion Fab. Zeitschrift für die gesammten Naturwissenschaften 46: 421-438.

Thunberg C.P. 1822. Ichneumonoidea, Insecta Hymenoptera illustrate. Mémoires de l'Académie Impériale des Sciences de Saint Petersbourg 8: 249-281.

Tosquinet J. 1896. Contributions à la faune entomologique de l'Afrique. Ichneumonides. Mémoires de la Société entomologique de Belgique 5: 1-430.

Townes H.K. 1971. The genera of Ichneumonidae, Part 4. Memoirs of the American Entomological Institute 17: 1-372.

Townes H.K. \& Townes M. 1973. A catalogue of the Ethiopian Ichneumonidae. Errata for 1944-1945 Nearctic catalogue, 1965 eastern Palearctic catalogue and 1966 Neotropic catalogue. Memoirs of the American Entomological Institute 9: 1-416.

van Noort S. 2004. Ichneumonid (Hymenoptera: Ichneumonoidea) diversity across elevation gradient on Mounts Doudou in Southwestern Gabon. California Academy of Sciences Memoir 28: 187-216.

Wahl D. 2014. Order Hymenoptera, family Ichneumonidae. Arthropod Fauna of the UAE 5: 341-406.

Wilkinson D.S. 1928. New parasitic Hymenoptera. Bulletin of Entomological Research 19: 261-265. https://doi.org/10.1017/S0007485300020599

Yu D.S., Achterberg C. van \& Horstmann K. 2012. Taxapad 2012, Ichneumonoidea 2011. Database on flash-drive. Ottawa/Ontario, Canada. Available from http://www.taxapad.com [accessed 27 Jan. 2016].

Zohary M. 1973. Geobotanical Foundations of the Middle East, Vols 1-2. Fischer, Stuttgart / Swets and Zeitlinger, Amsterdam.

Manuscript received: 17 July 2016

Manuscript accepted: 22 February 2017

Published on: 9 November 2017

Topic editor: Gavin Broad

Desk editor: Chloe Chester

Printed versions of all papers are also deposited in the libraries of the institutes that are members of the EJT consortium: Muséum national d'Histoire naturelle, Paris, France; Botanic Garden Meise, Belgium; Royal Museum for Central Africa, Tervuren, Belgium; Natural History Museum, London, United Kingdom; Royal Belgian Institute of Natural Sciences, Brussels, Belgium; Natural History Museum of Denmark, Copenhagen, Denmark; Naturalis Biodiversity Center, Leiden, the Netherlands; Museo Nacional de Ciencias Naturales-CSIC, Madrid, Spain; Real Jardín Botánico de Madrid CSIC, Spain. 
Appendix 1. Collection sites in Saudi Arabia. Alt.= altitude; LT = light trap; MT = Malaise trap.

\section{Al Baha}

Shada Al Ala

LT 1: $19^{\circ} 50.575^{\prime} \mathrm{N} 41^{\circ} 18.691^{\prime} \mathrm{E}$, alt. $1666 \mathrm{~m}$.

LT 2: $19^{\circ} 50.411^{\prime} \mathrm{N}, 41^{\circ} 18.686^{\prime} \mathrm{E}$, alt. $1611 \mathrm{~m}$.

LT 3: $19^{\circ} 50.329^{\prime} \mathrm{N}, 41^{\circ} 18.604^{\prime} \mathrm{E}$, alt.1563 m.

LT 4: $19^{\circ} 50.710^{\prime} \mathrm{N}, 41^{\circ} 18.267^{\prime} \mathrm{E}$, alt. $1474 \mathrm{~m}$.

LT 5: $19^{\circ} 51.066^{\prime} \mathrm{N}, 41^{\circ} 18.037^{\prime} \mathrm{E}$, alt. $1325 \mathrm{~m}$.

LT 6: $19^{\circ} 51.762^{\prime} \mathrm{N}, 41^{\circ} 18.089^{\prime} \mathrm{E}$, alt.1225 m.

LT House: $19^{\circ} 52.598^{\prime} \mathrm{N}, 41^{\circ} 18.672^{\prime} \mathrm{E}$, alt. $892 \mathrm{~m}$.

Wadi Neera: $19^{\circ} 44.870^{\prime} \mathrm{N}, 41^{\circ} 18.267^{\prime} \mathrm{E}$, alt. $471 \mathrm{~m}$.

Thee Ain: $19^{\circ} 55.774^{\prime} \mathrm{N}, 41^{\circ} 26.574^{\prime} \mathrm{E}$, alt. $741 \mathrm{~m}$.

Wadi Turubah: $20^{\circ} 14.4^{\prime} \mathrm{N}, 41^{\circ} 15.2^{\prime} \mathrm{E}$, alt. $1842 \mathrm{~m}$.

Wadi Ghanuna: $19^{\circ} 25.568^{\prime} \mathrm{N}, 41^{\circ} 36.548^{\prime} \mathrm{E}, 366 \mathrm{~m}$.

\section{Asir}

Abha, Raydah

LT 1: $18^{\circ} 12.265^{\prime} \mathrm{N}, 42^{\circ} 24.744^{\prime} \mathrm{E}$, alt. $2820 \mathrm{~m}$.

LT 2: $18^{\circ} 12.315^{\prime} \mathrm{N}, 42^{\circ} 24.607^{\prime} \mathrm{E}$, alt. $2761 \mathrm{~m}$.

LT 3: $18^{\circ} 12.095^{\prime} \mathrm{N}, 42^{\circ} 24.536^{\prime} \mathrm{E}$, alt. $2578 \mathrm{~m}$.

LT 4: $18^{\circ} 11.884^{\prime} \mathrm{N}, 42^{\circ} 24.435^{\prime} \mathrm{E}$, alt. $2387 \mathrm{~m}$.

LT 5 and MT 5: $18^{\circ} 11.766^{\prime} \mathrm{N}, 42^{\circ} 24.315^{\prime} \mathrm{E}$, alt. $2285 \mathrm{~m}$.

LT 6: $18^{\circ} 11.695^{\prime} \mathrm{N}, 42^{\circ} 23.818^{\prime} \mathrm{E}$, alt. $1897 \mathrm{~m}$.

LT 7: $18^{\circ} 11.679^{\prime} \mathrm{N}, 42^{\circ} 23.691^{\prime} \mathrm{E}$, alt. $1851 \mathrm{~m}$.

LT 8 and MT 8: $18^{\circ} 11.618^{\prime} \mathrm{N}, 42^{\circ} 23.420^{\prime} \mathrm{E}$, alt. $1772 \mathrm{~m}$.

LT 9: $18^{\circ} 11.749^{\prime} \mathrm{N}, 42^{\circ} 23.345^{\prime} \mathrm{E}$, alt. $1614 \mathrm{~m}$.

LT House: $18^{\circ} 13.347^{\prime} \mathrm{N}, 42^{\circ} 24.133^{\prime} \mathrm{E}$, alt. $2717 \mathrm{~m}$.

Abha, Wadi Rida: $18^{\circ} 11.71^{\prime} \mathrm{N}, 42^{\circ} 22.081^{\prime} \mathrm{E}$, alt. $1656 \mathrm{~m}$.

Abha, Tamniah Dam: $18^{\circ} 01.353^{\prime} \mathrm{N}, 42^{\circ} 45.814^{\prime} \mathrm{E}$, alt. $2301 \mathrm{~m}$.

Al-Magardah, Wadi Yabah: $19^{\circ} 14.911^{\prime} \mathrm{N}, 41^{\circ} 47.225^{\prime} \mathrm{E}$, alt. $402 \mathrm{~m}$.

K. Mushayt, Wadi Bisha: $18^{\circ} 20.018^{\prime} \mathrm{N}, 42^{\circ} 42.215^{\prime} \mathrm{E}$, alt. $1990 \mathrm{~m}$.

Wadi Sabean: $17^{\circ} 52.577^{\prime} \mathrm{N}, 42^{\circ} 16.681^{\prime} \mathrm{E}$, alt. $766 \mathrm{~m}$.

\section{Riyadh}

Al Quwayiyah, Rawdet Al Harmalyiah

LT 1: $24^{\circ} 18.572^{\prime} \mathrm{N}, 45^{\circ} 09.993^{\prime} \mathrm{E}$, alt. $774 \mathrm{~m}$.

LT 3: $24^{\circ} 18.572^{\prime} \mathrm{N}, 45^{\circ} 09.993^{\prime} \mathrm{E}$, alt. $774 \mathrm{~m}$.

MT.4: $24^{\circ} 18.372^{\prime} \mathrm{N}, 45^{\circ} 10.776^{\prime} \mathrm{E}$, alt. $769 \mathrm{~m}$.

LT 5: $24^{\circ} 20.226^{\prime} \mathrm{N}, 45^{\circ} 09.256^{\prime} \mathrm{E}$, alt. $774 \mathrm{~m}$.

Al Waseel: $24^{\circ} 48.753^{\prime} \mathrm{N}, 46^{\circ} 30.745^{\prime} \mathrm{E}$, alt. $660 \mathrm{~m}$.

Al Aflag, Rawdet Farshet Sheaal

LT 1: $22^{\circ} 24.161^{\prime} \mathrm{N}, 46^{\circ} 35.547^{\prime} \mathrm{E}$, alt. $588 \mathrm{~m}$.

LT 10: $22^{\circ} 23.725^{\prime} \mathrm{N}, 46^{\circ} 34.847^{\prime} \mathrm{E}$, alt. $589 \mathrm{~m}$.

LT 11: $22^{\circ} 23.828^{\prime} \mathrm{N}, 46^{\circ} 34.998^{\prime} \mathrm{E}$, alt. $583 \mathrm{~m}$.

Al Aflag, Wadi Ghaihab: $22^{\circ} 19.392^{\prime} \mathrm{N}, 46^{\circ} 26.128^{\prime} \mathrm{E}$, alt. $640 \mathrm{~m}$.

Al Amaryiah: $24^{\circ} 51.576^{\prime} \mathrm{N}, 46^{\circ} 55.753^{\prime} \mathrm{E}$, alt. $630 \mathrm{~m}$.

Deirab: $24^{\circ} 30.367^{\prime} \mathrm{N}, 46^{\circ} 37.166^{\prime} \mathrm{E}$, alt. $645 \mathrm{~m}$.

Al Kharg: $24^{\circ} 30.367^{\prime} \mathrm{N}, 46^{\circ} 37.166^{\prime} \mathrm{E}$, alt. $645 \mathrm{~m}$.

Education Farm: $24^{\circ} 43.83^{\prime} \mathrm{N}, 46^{\circ} 37.216^{\prime}$ E, alt. $689 \mathrm{~m}$. 
Huraymila: $25^{\circ} 07.666^{\prime} \mathrm{N}, 46^{\circ} 05.283^{\prime} \mathrm{E}$, alt. $785 \mathrm{~m}$.

Muzahimiyah, Al Khararah: $24^{\circ} 23.01^{\prime} \mathrm{N}, 46^{\circ} 14.14^{\prime} \mathrm{E}$, alt. $650 \mathrm{~m}$.

Rhodet Khorim: $25^{\circ} 25.943^{\prime} \mathrm{N}, 47^{\circ} 13.863^{\prime} \mathrm{E}, 572 \mathrm{~m}$.

Ummul Hammam: $24^{\circ} 41.330^{\prime} \mathrm{N}, 46^{\circ} 39.327^{\prime} \mathrm{E}$, alt. $633 \mathrm{~m}$.

Wadi Hanifah

LT (no number): $24^{\circ} 54.326^{\prime} \mathrm{N}, 46^{\circ} 11.325^{\prime} \mathrm{E}$, alt. $814 \mathrm{~m}$.

LT 3: $24^{\circ} 54.421^{\prime} \mathrm{N}, 46^{\circ} 10.902^{\prime} \mathrm{E}$, alt. $809 \mathrm{~m}$.

Appendix 2. COI marker registration numbers (BOLD Systems database).

\begin{tabular}{lccc}
\hline Species & Length (bp) & Sequence page & Barcode Index Number Registry \\
\hline Enicospilus arabicus sp. nov. & 573 & OPSAU004-16 & BOLD:ADB3498 \\
Enicospilus brevicornis & 575 & OPSAU009-16 & BOLD:ADB3412 \\
Enicospilus capensis & 629 & OPSAU011-16 & NA \\
Enicospilus divisus & 608 & OPSAU015-16 & BOLD:ADB4430 \\
Enicospilus dubius & 582 & OPSAU005-16 & BOLD:ADB4296 \\
Enicospilus grandiflavus & 632 & OPSAU013-16 & NA \\
Enicospilus mirabilis sp. nov. & 625 & OPSAU010-16 & BOLD:ADB4082 \\
Enicospilus nervellator & 610 & OPSAU012-16 & BOLD:ADB4509 \\
Enicospilus nervellator & 621 & OPSAU016-16 & BOLD:ADB4509 \\
Enicospilus oculator & 570 & OPSAU007-16 & BOLD:ADB4114 \\
Enicospilus odax & 580 & OPSAU001-16 & BOLD:ADB4115 \\
Enicospilus oweni & 605 & OPSAU002-16 & BOLD:ADB4116 \\
Enicospilus pacificus & 565 & OPSAU008-16 & BOLD:AAV0745 \\
Enicospilus pallidus & 577 & OPSAU014-16 & NA \\
Enicospilus pseudoculator sp. nov. & 579 & OPSAU017-16 & BOLD:ADB4338 \\
Enicospilus rundiensis & 577 & OPSAU006-16 & BOLD:ADB5118 \\
Enicospilus shadaensis sp. nov. & 635 & OPSAU003-16 & BOLD:ABX3540 \\
\hline
\end{tabular}

